(B) Lawrence Berkeley Laboratory UNIVERSITY OF CALIFORNIA
Materials \& Chemical Sciences Division

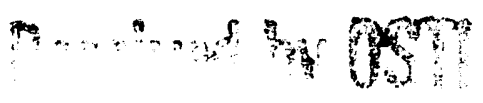

OE:17991

Quantum-Limited Detection of Millimeter Waves

Using Superconducting Tunnel Junctions

C.A. Mears

(Ph.D. Thesis)

September 1991

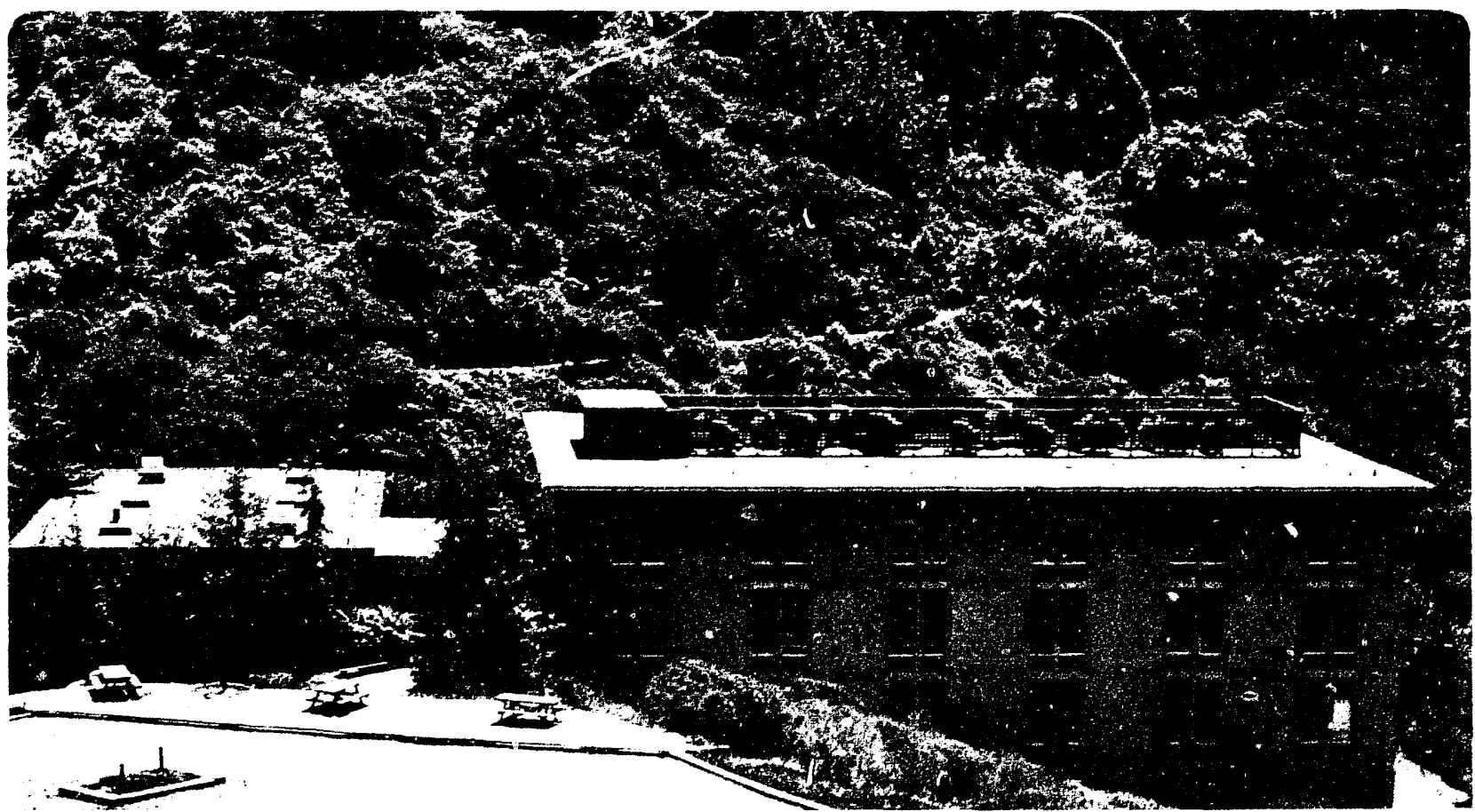

Prepared for the U.S. Department of Energy under Contract Number DE-AC03-76SF00098 


\section{DISCLAIMER}

This document was prepared as an account of work sponsored by the United States Government. Neither the United States Government nor any agency thereof, nor The Regents of the University of California, nor any of their eniployees, makes any warranty, express or implied, or assumes any legal liability or responsibility for the accuracy, completeness, or usefulness of any information, apparatus, product, or process disclosed, or represents that its use would not infringe privately owned rights. Reference herein to any specific commercial product, process, or service by its trade name, trademark, manufacturer, or otherwise, does not necessarily constitute or imply its endorsement, recommendation, or favoring by the United States Government or any agency thereof, or The Regents of the L'niversity of California. The views and opinions of authors expressed herein do not necessarily state or reflect those of the United States Government or any agency thereof or The Regents of the University of California and shall not be used for advertising or product endorsement purposes.

Lawrence Berkeley Laboratory is an equal opportunity employer. 
LBL --31391

DE92 004757

\title{
Quantum-Limited Detection of Millimeter Waves Using Superconducting Tunnel Junctions
}

\author{
C.A. Mears \\ Ph.D. Thesis \\ Physics Department \\ University of California \\ and \\ Materials Sciences Division \\ Lawrence Berkeley Laboratory \\ University of California \\ Berkeley, CA 94720
}

September 1991

This work was supported in part by the Department of Defense and by the Director, Office of Energy Research, Office of Basic Energy Sciences, Materials Sciences Division, of the U.S. Department of Energy under Contract No. DE-AC03-76SF00098. 


\title{
Quantum-Limited Detection of Millimeter Waves \\ Using Superconducting Tunnel Junctions
}

\author{
By
}

\section{Carl Atherton Mears}

\begin{abstract}
The quasiparticle tunneling current in a superconductor-insulatorsuperconductor (SIS) tunnel junction is highly nonlinear. Such a nonlinearity can be used to mix two millimeter wave signals to produce a signal at a much lower intermediate frequency. We have constructed several millimeter and sub-millimeter wave SIS mixers in order to study high frequency response of the quasiparticle tunneling current and the physics of high frequency mixing. We have made the first measurement of the out-of-phase tunneling currents in an SIS tunnel junction. We have developed a method that allows us to determine the parameters of the high frequency embedding circuit by studying the details of the pumped I$\mathrm{V}$ curve. We have constructed a $80-110 \mathrm{GHz}$ waveguide-based mixer test apparatus that allows us to accurately measure the gain and added noise of the SIS mixer under test. Using extremely high quality tunnel junctions, we have measured an added mixer noise of $0.61 \pm 0.36$ quanta, which is within 25 percent of the quantum limit imposed by the Heisenberg uncertainty principle. This measured performance is in excellent agreement with that predicted by Tucker's theory of quantum mixing. We have also studied quasioptically coupled millimeter- and submillimeterwave mixers using several types of integrated tuning elements.
\end{abstract}




\section{CONTENTS}

1. Introduction ................................................................... 1

2. Theoretical Background............................................................ 3

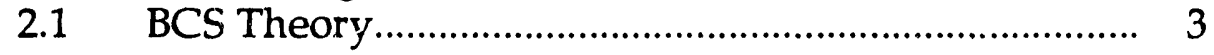

2.2 Superconducting Tunnel Jurictions............................ 3

2.3 High Frequency Response.......................................... 5

2.4 Mixer Theory............................................................ 7

2.5 The Quantum Limit..................................................... 13

3. Measurement of the Quantum Susceptance......................... 15

$3.1 \quad$ Introduction............................................................... 15

3.2 Theoretical Background............................................. 17

3.3 Experimental Details.................................................... 22

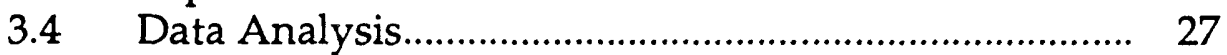

3.5 Discussion.................................................................. $\quad 30$

4. Analysis of Pumped I-V curves............................................. 32

4.1 Introduction.................................................................... 32

4.2 Photon-Assisted Tunneling..................................... 32

4.3 I-V Curve with Arbitrary Source Admittance.......... 33

4.4 Fitting of Experimental Pumped I-V Curves............ 35

4.5 Conclusions.................................................................. 39

5. Waveguide Mixer Measurements.......................................... 40

5.1 Introduction.................................................................. 40

5.2 RF Measurement Apparatus...................................... 40

5.3 IF Measurement Apparatus........................................... 42

5.4 dc Measurement Apparatus........................................... 42

5.5 Tantalum Junctions..................................................... 42

5.6 Measurement Scheme................................................ 44

5.7 Mixer Optimization.................................................... 46

5.8 Mixer Performance..................................................... 46

5.9 Comparison with Theory.............................................. 46

6. Measurement of Quasioptically Coupled SIS Mixers........... 52

6.1 Introduction.................................................................. 52

$6.2 \quad$ Mixer Test Apparatus.................................................... 53

6.3 Integrated Tuning Elements....................................... 55

6.4 Test of Tuning elements using FTS............................. 56

6.5 Design and Measurement of Tuning Elements......... 58

6.6 Conclusion....................................................................... 63

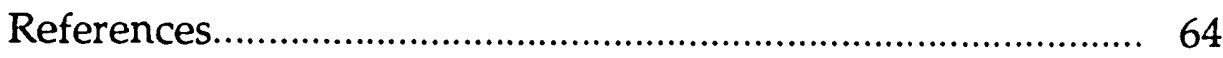




\section{ACKNOWLEDGEMENTS}

It seems that it is traditional at this point to thank all the people that made this dissertation possible. First, I'd like to thank all the people I worked with during my tenure here in the Richards Group. People that deserve special mention (and are hereby placed in the SIS mixer hall of fame) are: Simon Verghese, for forcing me to learn some physics so that I could keep up with his wild speculations. Qing $\mathrm{Hu}$, for giving me muchneeded periodic abuse, and teaching me much of what I know about superconducting junctions. Gary Bernstein, for introducing me to so much great music, as well as teaching me about the makeup of manganin. Tom Kenny, for pointing out the virtues of Arnold Schwarzenegger and Bmovies. Mike Nahum, for being a endless source of unanswerable questions, as well as some very strange noises. John Birmingham, for always understanding my obscure literary allusions. David Miller, for teaching me how to use the Fourier Spectrometer with his characteristic generosity. I'd also like to thank all Helium Czars past and present for their great gift to society.

Of course, I'd like to thank my advisor, Paul Richards. He provided much needed kicks in the behind, nudges in fruitful directions, countless pieces of essential information, soldering lessons, funding, physical intuition, as well as the molding forces that transformed me from a ragclad, snot-nosed kid to someone who can pass, if required, as a professional member of the research community.

I'd also like to thank my housmates at the Ward Street House over the years, especially the one's who regularly cooked me dinner. These include R. E. (the 'Scoon) Thomson, Bob (Euro)Phelps, Mats Gustafson, Andre (The Bubwon) Clapp, and Margot Damaser. Discussions around the dinner table were always, shall we say, spirited and provided a much needed diversion from the "rigors of grad school life." Also providing an essential diversion was the Cal Ultimate Frisbee team. I don't think I would have made it 'til now without having a healthy outlet for my agressive tendencies I made a lot of good friends on the ultimate field. These include Marla Feller, who was always willing talk about anything over coffee, Steve (The Bogus One) Weiss, who taught me that camping and comfort can be synonomous, Eric (Bubba MC Hockeypuck) Gingold, always ready to recite some Public Enemy, Jeff (The Buddha Bubba) Soller who always wants to have fun, and is probably having some right now, and Panna Lossy, who convinced me to trust my feet.

Special thanks goes to Mari Strain, who has been a wonderful companion these last few months (and who I would never ask to type this inanuscript).

Special thanks also goes to my first advisors, my parents. Without the preparation and support I received from them, I would have never gone to graduate school. I was essentially raised form birth to be an experimental physicist. 


\section{Chapter 1 \\ Introduction}

The quasiparticle tunneling current in a superconductor-insulatorsuperconductor (SIS) tunnel junction is highly nonlinear. There is an extremely rapid onset of tunneling current at a voltage corresponding to the sum of the energy gaps of the superconductors on either side of the junction. Such a nonlinearity can be used to mix two millimeter wave signals; a strong local oscillator at frequency $\omega_{\mathrm{LO}}$ and a weaker signal at frequency $\omega_{S}$ to produce a signal at a lower or intermediate frequency $\omega_{\text {IF }}$. An SIS tunnel junction can thus be used as the mixer in a millimeter wave heterodyne reciever. At frequencies higher than about $30 \mathrm{GHz}$ where it is difficult to build sensitive amplifiers, a mixer is typically the first element in the receiver. In Fig. 1.1 we show a block diagram of a typical high frequency receiver. Usually, $\omega_{I F}$ is much less than $\omega_{\mathrm{LO}}$ and $\omega_{\mathrm{S}}$, so $\omega_{\mathrm{IF}}$ is low enough so that it is possible build a sensitive intermediate frequency amplifier.

The important parameters that characterize a mixer are the conversion gain $G_{m}$ and the spectral density of the added noise $S_{m}$. The conversion gain is defined as the ratio of the output power of the mixer at the intermediate frequency to the available power input at the signal frequency. The added noise is refered to the input of the mixer. Traditionally this has been expressed as the equivalent temperature (noise temperature) of a blackbody placed at the input to account for the added noise. For a typical receiver, $\mathrm{S}_{\mathrm{m}}$ $\gg \hbar \omega_{s}$, and thus $T_{m}=S_{m} / k_{B}$. However, an SIS mixer can have noise levels low enough that $S_{m} \sim \hbar \omega_{s}$. In this regime, the different definitions of noise temperature used by various authors yield different results. To avoid this ambiguity, we report values of $S_{m}$ measured in units of quanta $\left(\hbar \omega_{s}\right)$ of the signal radiation.

As we can see in Fig 1.1, a receiver contains an intermediate frequency amplifier with added noise of spectral density $\mathrm{S}_{\mathrm{IF}}$. The spectral density of the overall noise added by the receiver is given by

$$
\mathrm{S}_{\mathrm{r}}=\mathrm{S}_{\mathrm{m}}+\mathrm{S}_{\mathrm{IF}} / \mathrm{G}_{\mathrm{m}} .
$$

In order for the receiver to have good overall noise performance, it is necessary to make $S_{m}$ and $S_{i f}$ small and $G_{m}$ as large as is practical.

Because the voltage scale of the nonlinearity of the SIS junction is smaller than the voltage associated with a photon of millimeter wave radiation $\hbar \omega_{s}$, we must use a quantum theory to predict its high frequency response. The quantum theory was worked out by Tucker. 1,2 The Tucker theory makes several surprising and important predictions. First, the conversion gain $G_{m}$ can be greater than unity. ${ }^{3}$ This was unexpected since classically $\mathrm{G}_{\mathrm{m}} \leq 1.0$. The theory also predicts that the added noise can 
approach the quantum limit, i.e. be limited only by the $\Delta \phi \Delta \mathrm{n}$ uncertainty relation.

SIS heterodyne receivers are currently the most sensitive coherent receivers over a broad range of the millimeter and sub-millimeter electromagnetic spectrum. ${ }^{4}$ However, even the best of these receivers have fallen short of the performance predicted by the Tucker theory. Because of the lack of detailed comparison between experimental and theoretically calculated performance, it has been unclear whether this discrepancy arises

from difficulties in coupling the signal to the mixer, or from problems with Tucker's theory.

The purpose of this work is to provide a detailed comparison between the predictions of the Tucker theory and experimental measurements of the millimeter wave behavior of SIS tunnel junctions. The following outline provides a framework for our investigations. In Chapter 2 we review relevant details of the BCS theory of superconductivity, especially as it applies to quasiparticle tunneling. We discuss Werthamer's ${ }^{5}$ derivaton of the highfrequency response function for an SIS junction and introduce the Tucker theory of quantum mixing. We also discuss the quantum noise limit as it applies to mixers. In Chapter 3 we discuss measurements of the high frequency response of a SIS tunnel junction in the small signal limit. We have made the first measurement of the out-of-phase component of the quasiparticle tunneling current. ${ }^{6,7}$ This out-of-phase current is roughly analogous to the $\cos \phi$ term in the Josephson or pair tunneling current. In Chapter 4 we use the Tucker theory to explain the dependence of the shape of the pumped I-V curve on embedding admittance seen by the junction. ${ }^{8} \mathrm{We}$ have fit the shapes of experimentally measured pumped I-V curves to deduce the value of the high-frequency embedding admittance under the actual experimental conditions. ${ }^{9,} 10$ In Chapter 5 we discuss extremely accurate mixer measurements using high quality tunnel junctions. ${ }^{9,} 10$ These measurements were carried out in a waveguide-based mixer test apparatus specially designed to perform accurate measurements of mixer noise and gain. We have measured the lowest added mixer noise reported to date. Measured performance is in excellent agreement with that predicted by the Tucker theory. This represents the first detailed comparison between experimentally measured noise performance and the Tucker theory. In Chapter 6 we discuss the the development of a quasioptically-coupled submillimeter-wave receiver. ${ }^{11}$ This receiver utilizes planar lithographed antennas to couple the radiation to the mixer, as well as several types of integrated tuning elements to resonate the capacitance of the junction. 


\section{Chapter 2 \\ Theoretical Overview}

This chapter provides the basic theoretical background necessary for understanding the discussion of high-frequency response of tunnel junctions and the discussion of quasiparticle mixers operated in the quantum limit that is presented in the following chapters. First we discuss the BCS theory of superconductivity, focusing on those aspects related to superconducting tunnel junctions. We then discuss tunneling between superconductors, including the treatment of high-frequency response worked out by Werthamer. We then turn our attention to Tucker's quantum theory of mixing, and discuss its remarkable predictions. We finish this chapter with a discussion of quantum limited sensitivity. This minimum added noise in the mixing process is imposed by the Heisenberg uncertainty principle.

\subsection{BCS Theory}

Excellent treatments of the BCS Theory of superconductivity already exist in a number of books, ${ }^{12}$ as well in the original work, ${ }^{13}$ so only the results essential to understanding quasiparticle unneling are presented here. The basic idea of the BCS theory is that an attractive interaction exists between two electrons due to an exchange of a virtual phonon. Because of this interaction, the Fermi sea of electrons is unstable to the formation of bound pairs of electrons (Cooper pairs) with roughly equal and opposite momentum. Below a critical temperature, a new ground state consisting of these Cooper pairs is formed - the superconducting state. Excitations above this ground state are called quasiparticles. There are no low-lying quasiparticles, as all quasiparticles have an energy of at least $\Delta$, the superconducting energy gap. The density of quasiparticle states diverges at the gap energy. This divergence is the cause of the extreme nonlinearity in the quasiparticle currents in a superconducting tunnel junction.

\section{Superconducting Tunnel Junctions}

Since the discovery of tunneling between superconductors, tunneling phenomena have been studied extensively. SIS tunnel junctions exhibit tunneling currents due both to the tunneling of Cooper pairs, or Josephson tunneling, and to the tunneling of single-particle excitations, or quasiparticle tunneling. Josephson tunneling has been studied extensively, and is the basis of the SQUID (Superconducting QUantum Interference Device), several types of digital logic circuits, and studies of nacroscopic quantum phenomena. The focus of the work presented in this thesis is the extremely nonlinear tunneling currents due to the divergence in the quasiparticle density of states discussed in section 2.1. We now discuss the physical origin of this nonlinear I-V curve. 
Following the treatment by Cohen, Falicov and Phillips, ${ }^{14}$ we use a Hamiltonian theory to describe the quasiparticle tunneling through a potential barrier. The Hamiltonian used is

$$
\mathrm{H}=\mathrm{H}_{\mathrm{L}}+\mathrm{H}_{\mathrm{R}}+\mathrm{H}_{\mathrm{T}}+\mathrm{eV}(\mathrm{t}) \mathrm{N}_{\mathrm{L}},
$$

where $H_{L}$ and $H_{R}$ are the many-body Hamiltonians describing the left and right electrode respectively. $\mathrm{V}(\mathrm{t})$ is the applied voltage, and $\mathrm{N}_{\mathrm{L}}$ is the number operator for the left side,

$$
\mathrm{N}_{\mathrm{R}}=\sum_{\mathrm{k}} \mathrm{c}_{\mathrm{k}} \mathrm{c}_{\mathrm{k}} \text {. }
$$

$\mathrm{H}_{\mathrm{T}}$ describes the transfer, by tunneling, of a quasiparticle from one electrode to another and is given by

$$
\mathrm{H}_{\mathrm{T}}=\sum_{\mathrm{k}, \mathrm{q}}\left(\mathrm{T}_{\mathrm{kq}} \mathrm{c}_{\mathrm{k}} \mathrm{c}_{\mathrm{q}}^{+}+\mathrm{T}_{\mathrm{kq}}^{*} \mathrm{c}_{\mathrm{q}} \mathrm{c}_{\mathrm{k}}^{+}\right) \text {. }
$$

Here, $c_{k}\left(c_{q}\right)$ and $c_{k}{ }^{\top}\left(c_{q}{ }^{\dagger}\right)$ are the quasiparticle destruction and creation operators for the right (left) side electrode. The tunneling matrix elements $\mathrm{T}_{\mathrm{kq}}$ characterize the strength of the coupling between the superconducting electrodes. They are assumed to be small enough that $\mathrm{H}_{\mathrm{T}}$ may be analyzed using lowest order perturbation theory. A calculation of the tunneling current using linear response theory yields a dc quasiparticle current,

$$
\begin{array}{r}
I_{d c}(V)=\frac{2 \pi e}{\hbar^{2}} \sum_{k q \sigma} \int_{-}^{-} d \omega_{L} d \omega_{R}\left|T_{k q}\right|^{2} A_{L}\left(k, \omega_{L}\right) A_{R}\left(k, \omega_{R}\right) \\
{\left[f\left(\hbar \omega_{L}\right)-f\left(\hbar \omega_{R}\right)\right] \delta\left(e V / \hbar+\omega_{L}-\omega_{R}\right)}
\end{array}
$$

Here $f(\hbar \omega)=\left(e^{\hbar \omega / k T}+1\right)^{-1}$ is the Fermi-Dirac distribution function, and $A_{L, R}$ are the single particle spectral distribution functions for the left and right side electrodes. In the BCS theory, the spectral distribution function is given by

$$
A(k, \omega)=u_{k}^{2} \delta\left(\omega-E_{k} / \hbar\right)+v_{k}^{2} \delta\left(\omega+E_{k} / \hbar\right) .
$$

where $v_{k}^{2}=1-u_{k}^{2}=1 / 2\left(1-\epsilon_{k} / E_{k}\right)$ is the probability that a given pair $(k \uparrow$, $k \downarrow)$ is occupied in the BCS ground state. Here $\epsilon_{k}$ is the normal state energy of an excitation with momentum $\hbar k$, and $E_{k}=\left(\epsilon^{2}+\Delta^{2}\right)^{1 / 2}$ is the energy of an excitation of momentum $\hbar k$ in the superconducting state. $A(k, \omega)$ depends only on $u_{k}{ }^{2}$ and $v_{k}{ }^{2}$, so no "coherence factors" of $u_{k} v_{k}$ enter into the 
tunneling current. If we substitute the spectral distribution function (Eq. 2.6) into the expression for the tunneling current (Eq. 2.5), and rewrite everything in terms of $E_{k}$, we obtain the simple expression,

$$
\begin{aligned}
I_{d c, S I S}(V)=\frac{1}{e R_{N}} & \int_{-\infty}^{\infty} \frac{\left|E^{\prime}\right|}{\left(E^{\prime 2}-\Delta_{1}^{2}\right)^{1 / 2}} \frac{\left|E^{\prime}-e V\right|}{\left[\left(e V-E^{\prime}\right)^{2}-\Delta_{2}^{2}\right]^{1 / 2}} \\
& \times\left[f\left(E^{\prime}-e V\right)-f\left(E^{\prime}\right)\right] d E^{\prime}
\end{aligned}
$$

Note that we have assumed that $T_{k q}$ is constant and absorbed it into $R_{N}$, the normal resistance of the junction. Because no coherence terms are present in the expression for the tunneling current, we are able to use a simple "semiconductor" model for discussion. In Fig. 2.1, we plot the density of states for single-particle excitations $D(E)$ as a function of energy for each electrode. At $\mathrm{T}=0$, there are no thermally excited quasiparticles, and there is an energy gap $2 \Delta$ for each electrode required to break a Cooper pair to produce two quasiparticle excitations. As we increase the voltage $V$, no tunneling takes place until $\mathrm{eV}=\Delta_{1}+\Delta_{2}$. Here there is sufficient energy to allow an electron to tunnel into an empty quasiparticle state above the gap on the right, leaving behind an unpaired electron quasiparticle on the left. Because of the singularity of the density of states, the onset of tunneling current is extremely rapid. Above the sum-gap voltage $V_{g}=\left(\Delta_{1}+\Delta_{2}\right) / e, I_{d} c$ asymptotically approaches $V / R_{N}$, where $R_{N}$ is the normal resistance. $I_{d c}, S I S$ is plotted for several reduced temperatures $t=T / T_{C}$ in Fig 2.1b. Note that for $t$ near 1 there is also a structure at the difference-gap voltage $\left|\Delta_{1}-\Delta_{2}\right| /$ e due to thermally excited quasiparticles.

In an ideal junction, the current rise at the sum-gap voltage is infinitely sharp. In real junctions, the current rise is rounded by effects such as short quasiparticle lifetimes, gap anisotropy, or gap inhomogeneity.

\subsection{High-Frequency Response of Tunnel Junctions}

Based on a perturbation theory using the tunneling Hamiltonian, ${ }^{14-18}$ Werthamer ${ }^{5}$ derived an expres:ion for the tunneling current as a function of time in the presence of both $\mathrm{dc}$ and ac bias:

$$
\begin{aligned}
& I(t)=\operatorname{Im} \iint d \omega d \omega^{\prime}\left[W(\omega) W^{*}(\omega) e^{-i(\omega-\omega) t} j_{q p}\left(\omega^{\prime}+e V_{o} / \hbar\right)+\right. \\
& \left.+W(\omega) W(\omega) e^{-i(\omega+\omega) t+i \phi} j_{p}\left(\omega^{\prime}+e V_{o} / \hbar\right)\right]
\end{aligned}
$$

where $j_{q p}$ and $j_{p}$ are the response functions of quasiparticles and Cooper pairs respectively. The first term in Eq. (2.8) is the quasiparticle tunneling current. 
The second term is the pair tunneling current which depends on the phase difference $\phi$ between the superconducting ground state wave functions on the two sides of the junction. The real parts of the response functions correspond to the reactive components, and the imaginary parts correspond to the resistive components. $W(\omega)$ is the Fourier transform of the time-varying phase factor $W(t)$ caused by the ac bias voltage:

$W(t)=\exp \left\{-i \frac{e}{\hbar} \int^{t} d t^{\prime}\left[V\left(t^{\prime}\right)-V_{0}\right]\right\}=\int_{-\infty}^{\infty} d \omega W(\omega) e^{-i \omega t}$

For BCS-like superconductors, jqp and $j_{p}$ can be calculated using the density of states of quasiparticles and Cooper pairs. However, the calculation is quite complicated. ${ }^{5}$ The following shows that the quasiparticle response function jqp can be measured directly from the dc I-V curve. When the bias voltage $V(t)$ contains only a dc component $V_{0}$, then $W(\omega)=\delta(0)$, and from Eq. (2.8) we have,

$$
I(t)=\operatorname{Im}\left[j_{q p}\left(\omega_{0}\right)\right]+\operatorname{Re}\left[j_{p}\left(\omega_{0}\right)\right] \sin \phi+\operatorname{Im}\left[j_{p}\left(\omega_{0}\right)\right] \cos \phi,
$$

where $\omega_{0}=e V_{0} / \hbar$. Since both the $\sin \phi$ and $\cos \phi$ terms oscillate at the Josephson frequency $\omega_{j}=2 \mathrm{eV}_{\mathrm{o}} / \hbar$, the only dc component in Eq. (2.10) is the first term. Therefore, $\left.\operatorname{Imlj} \mathrm{qp}_{\mathrm{p}}\left(\mathrm{eV}_{\mathrm{o}} / \hbar\right)\right]$ is equal to the dc quasiparticle $\mathrm{I}-\mathrm{V}$ curve $\mathrm{I}_{\mathrm{dc}}\left(\mathrm{V}_{\mathrm{o}}\right)$,

$$
\operatorname{Im}\left[j_{q p}\left(\omega_{o}\right)\right]=I_{d c}\left(V_{o}\right)
$$

Eq. (2.11) implies that the imaginary part of the quasiparticle response function at frequency $\omega_{0}=e V_{0} / \hbar$ is equal to the dc tunneling current at bias voltage $V_{0}$. Because of the absence of $\operatorname{Re}\left[j_{q p}\left(\omega_{0}\right)\right]$ in Eq. (2.10), it is clear that the reactive part of the quasiparticle response function has no contribution to the tunneling current when the bias voltage is time-independent. In contrast to the quasiparticle response function, both the real and imaginary parts of the pair response function contribute to the tunneling current at $\mathrm{dc}$ bias. The real part of $j_{p}$ gives rise to the familiar Josephson $\sin \phi$ term, while the imaginary part of $j p$ gives the Josephson $\cos \phi$ term.

The real and imaginary parts of both quasiparticle and Cooper pair response functions are related through a frequency Kramers-Kronig transform, as required by any causal, finite response. For $\mathrm{j}_{\mathrm{qp}}(\omega){ }^{1}$ 


$$
\begin{gathered}
\operatorname{Re}\left[j_{q p}(\omega)\right]=P \int_{-}^{-} \frac{d \omega^{\prime}}{\pi} \frac{\operatorname{Im}\left[j_{q p}\left(\omega^{\prime}\right)\right]-\hbar \omega^{\prime} / e R_{n}}{\omega-\omega^{\prime}} \\
=I_{k k}(V)=P \int_{-}^{-} \frac{d V^{\prime}}{\pi} \frac{I_{d c}(V)-V^{\prime} / R_{n}}{V^{\prime}-V} .
\end{gathered}
$$

In Eq. (2.12), we have used Eq. (2.11) to replace $\operatorname{Im}\left[j_{q p}\left(\omega^{\prime}\right)\right]$ with $I_{d c}\left(V^{\prime}\right), e^{\prime} / \hbar=$ $\omega^{\prime}$ and $\mathrm{eV} / \hbar=\omega$. We subtract an Ohmic term from the quasiparticle I-V curve to prevent divergence of the integral. This is allowed because only the nonli ear portion of $\mathrm{I}_{\mathrm{dc}}(\mathrm{V})$ gives rise to a reactive component. The frequency-independent Ohmic response corresponds to an instantaneous current-voltage relation and thus does not contribute to the reactive component. It can be shown from Eq. (2.8) that all measurable quantities depend only on differences between values of $I_{K K}(V)$ and not on their absolute magnitudes. In Fig. 2.2(a) and 2.2(b), we plot an experimentally measured I-V curve of an SIS junction and the voltage Kramers-Kronig transform calculated from Eq. (2.12). The peak of $I_{k k}$ at the gap voltage $V_{g}$ corresponds to the sharp nonlinearity of the dc $\mathrm{I}-\mathrm{V}$ curve $\mathrm{I}_{\mathrm{dc}}(\mathrm{V})$ at $\mathrm{V}_{\mathrm{g}}$. At $\mathrm{T}=$ 0 , for an ideal SIS junction whose quasiparticle density of states is given by the BCS theory, the peak in $I_{k k}$ diverges logarithmically at $V_{g \cdot} 1,2,5,19-21$

Eqs. (2.11) and (2.12) suggest a very powerful way of deducing the frequency dependent response function of quasiparticles. The dc current $I_{d c}(V)$ as a function of dc bias voltage gives the imaginary part of the response function as a function of frequency; its voltage Kramers-Kronig transform gives the real part of the response function. Therefore the dc I-V curve, which can be easily measured, contains all the information about the response of the quasiparticles in an SIS junction at high frequencies. Two conditions must be satisfied for this statement to be valid. First, the quasiparticle tunneling must be elastic within the tunnel barrier so that the $\mathrm{dc}$ I-V curve gives direct information about the density of states of the quasiparticles in the two sides of the junction. Second, the tunneling probability must be small enough that tunneling does not significantly change the density of states on either side. These two conditions are met for SIS junctions with modest current densities $\leq 10^{4} \mathrm{~A} / \mathrm{cm}^{2}$, and high quality tunnel barriers which are free from impurities and imperfections.

\section{Mixer Theory}

\subsection{Basic Terminology}

Before discussing the quantum mixer theory, let us first introduce some basic mixer terminology. A mixer contains a non-linear element, in our case an SIS tunnel junction. The weak signal, at frequency $\omega_{s}$, and the the strong local oscillator, or "pump", at frequency $\omega_{\text {LO }}$ are coupled into the 
nonlinear device, where they produce an intermediate frequency $\omega_{\text {IF }}=\mid \omega_{s}-$

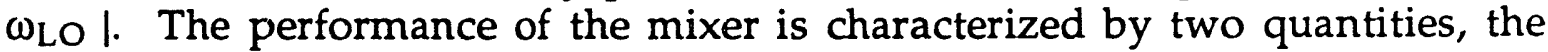
added noise referred to the input, and the conversion gain. The noise added by the mixer is characterized by the spectral density of the added noise referred to the input of the mixer. Traditionally, this has been reported as the temperature (the noise temperature) of a fictitious blackbody placed at the input of a noiseless, ideal mixer to account for the noise produced by the real mixer. This works well as long as the blackbody is in the Rayleigh-Jeans limit at the signal frequency. For an SIS mixer operating near the quantum limit, this is no longer true. Under these conditions, the definition of noise temperature becomes ambiguous. We choose to report the spectral density of the mixer noise in units of $\hbar \omega$ of the signal radiation.

The conversion gain is defined as:

$$
G_{m}=\frac{\text { power delivered to the IF Load }}{\text { available power at the signal frequency }} .
$$

The mixer will produce an output at $\omega_{\text {IF }}$ from either of two input frequencies, the upper sideband, at $\omega_{\mathrm{usb}}=\omega_{\mathrm{LO}}+\omega_{\mathrm{IF}}$, and the lower side band, at $\omega_{\text {lsb }}=\omega_{\text {LO }}-\omega_{\text {IF. }}$. Clearly, there are two distinct conversion gains, $G_{\text {usb }}$ and $\mathrm{G}_{\mathrm{lsb}}$ at the two frequencies. Sometimes equal powers are applied at the upper and lower side bands, in which case it is useful to define a double-sid band conversion gain $G_{d s b}=G_{l s b}+G_{u s b}$. This quantity is appropriate when the input signal is from a blackbody, as is the case when the mixer noise is being measured.

When the signal is in only in one sideband, that frequency is referred to as the "signal" frequency, and the other sideband is referred to as the "image" frequency. A mixer with $G_{\mathbf{s}} \gg G_{i}$ (image rejecting) is called a single-sideband (SSB) mixer. If $\mathrm{G}_{\mathrm{usb}}=\mathrm{G}_{\mathrm{lsb}}$, the mixer is called a doublesideband (DSB) mixer.

\subsubsection{Classical Mixer Theory}

The classical theory of mixers has been discussed by many authors. ${ }^{22,} 23$ Classical mixer theory, as well the quantum mixer theory that we will discuss later, uses the $Y$ matrix to describe the small signal mixing properties. The $Y-$ matrix is defined by

$$
i_{m}=\sum_{m^{\prime}} Y_{m m^{\prime}} v_{m^{\prime}}
$$

where

$$
v_{\text {sig }}=\operatorname{Re} \sum_{m=-\infty}^{\infty} v_{m} e^{i \omega_{m} t},
$$




$$
i_{\text {sig }}=\operatorname{Re} \sum_{m=-\infty}^{\infty} i_{m} e^{i \omega_{m} t}
$$

and

$$
\omega_{\mathrm{m}}=\mathrm{m} \omega_{\mathrm{LO}}+\omega_{\mathrm{IF}} \quad \mathrm{m}=0, \pm 1, \pm 2, \ldots .
$$

Note that $v_{\text {sig }}$ is only the small signal voltage, and does not contain the local oscillator, or pump, voltage. The $Y$-matrix describes how the nonlinear element, under the influence of the strong local oscillator signal, converts signals between the various frequencies, or ports, of the mixer. The equivalent circuit of the mixer that we use is shown in Fig. 2.3. The mth port of the mixier is assumed to be terminated by an embedding admittance $Y_{m}$. Signals are coupled to the mixer by currents sources in parallel with the $Y_{m}$ 's. Typically the mixer is operated with a signal injected at the $m=1$ (signal) port, and the output measured at the $m=0$ (IF) port, as shown in the figure.

If we know the $\mathrm{Y}$-matrix, analysis of the mixer is straightforward. For each port we have the equation,

$$
I_{m}=i_{m}+Y_{m} v_{m}=\sum_{m^{\prime}}\left(Y_{m m^{\prime}}+Y_{m} \delta_{m, m^{\prime}}\right) v_{m},
$$

which we invert to yield

$$
\mathrm{v}_{\mathrm{m}}=\sum_{\mathrm{m}^{\prime}} \mathrm{Z}_{\mathrm{mm}^{\prime}} \mathrm{I}_{\mathrm{m}^{\prime}}
$$

where

$$
Z=\left(Y+Y_{m} \delta_{m, m^{\prime}}\right)^{-1}
$$

When a signal source $I_{s}$ is placed at the $m=1$ port, the output voltage at the $\mathrm{m}=0$ (IF) port is given by

$$
\mathrm{v}_{0}=\mathrm{Z}_{00} \lambda_{01} \mathrm{I}_{\mathrm{s}} \text {, }
$$

where

$$
\lambda_{01}=\frac{Z_{01}}{Z_{00}} .
$$

It is easy to show that $\lambda_{01}$ is independent of $Y_{0}$, the IF load admittance. The SSD mixer gain is given by

$$
G_{m}=4 \operatorname{Re}\left(Y_{1}\right) \operatorname{Re}\left(Y_{0}\right)\left|Z_{01}\right|^{2}
$$

In the classical theory, the elements of the $\mathrm{Y}$-matrix are determined from the time-dependent modulation of the $\mathrm{dc} I-\mathrm{V}$ curve which produces a time-dependent conductance 


$$
G_{c l}(t)=\frac{d}{d V_{o}} I_{d c}\left(V_{o}+V_{L O C o s} \omega t\right)=\sum_{m=-\infty}^{\infty} G_{c l}(m \omega) e^{i m \omega t},
$$

which yields

$$
Y_{m m^{\prime}}^{\mathrm{c}}=G_{\mathrm{cl}}\left[\left(\mathrm{m}-\mathrm{m}^{\prime}\right) \omega\right] .
$$

The classical caiculaticn assumes that the high-frequency response of the junction is given by Eq. 2.24. As we have already seen in section 2.3, this is not the case when the frequency of the radiation is so high that $\hbar \omega / \mathrm{e}$ is larger than the voltage scale of the nonlinearity of the junction. Thus a quantum version of the $\mathrm{Y}$-matrix is required.

\subsubsection{Quantum Mixer Theory}

Tucker's quantum theory of mixing 1,2 describes a method of analyzing the performance of nonlinear resistive mixers where the voltage scale of the nonlinearity is small compared to $\hbar \omega / \mathrm{e}$. The theory assumes that the measured I-V curve of the device under study is entirely determined by elastic tunneling. This is a good assumption for high quality SIS tunnel junctions. The validity of this assumption will be discussed in more detail in chapter 5 .

In order to calculate the $\mathrm{Y}$-matrix using this theory, we must first determine the local oscillator waveform $V_{L O}(t)$ impressed across the device. In general this is a very difficult problem, both because it is difficult mathematically and also because it requires knowledge of the embedding admittance at all harmonics of the local oscillator frequency. Fortunately, for most practical mixers, we can use the 3-port approximation. In this approximation, we assume that all higher harmonics of the LO are shorted by the geometrical capacitance of the junction. In this case, $V_{L O}(t)=V_{\omega} \cos \omega t$. Also implicit in this assumption is that all signal ports with $|\mathrm{m}| \geq 2$ are also shorted, so that no voltages appear at these ports. Thus the only remaining ports are those with $m=1,0,-1$.

Once we know the magnitude $\mathrm{V}_{\omega}$ of the $\mathrm{LO}$ drive voltage, we can determine the elements $Y_{m m^{\prime}}=G_{m m^{\prime}}+i B_{m m^{\prime}}$ from 1,2 
and

$$
\begin{aligned}
G_{m m^{\prime}}= & \frac{e}{2 \hbar \omega_{m^{\prime}}} \sum_{n, n^{\prime}} J_{n}\left(\frac{e V_{\omega}}{\hbar \omega}\right) J_{n^{\prime}}\left(\frac{e V_{\omega}}{\hbar \omega}\right) \delta_{m-m^{\prime}} \delta_{n-n^{\prime}} \\
& {\left[I_{d c}\left(V_{o}+n^{\prime} \hbar \omega / e+\hbar \omega_{m^{\prime}}\right)-I_{d c}\left(V_{o}+n^{\prime} \hbar \omega / e\right)+\right.} \\
& \left.+I_{d c}\left(V_{o}+n \hbar \omega / e\right)-I_{d c}\left(V_{o}+n \hbar \omega / e-\hbar \omega_{m^{\prime}}\right)\right]
\end{aligned}
$$

$$
\begin{aligned}
B_{m m^{\prime}}= & \frac{e}{2 \hbar \omega_{m^{\prime}}} \sum J_{n, n^{\prime}}\left(\frac{e V_{\omega}}{\hbar \omega}\right) J_{n^{\prime}}\left(\frac{e V_{\omega}}{\hbar \omega}\right) \delta_{m-m^{\prime}} \delta_{n-n^{\prime}} \\
& {\left[I_{k k}\left(V_{o}+n^{\prime} \hbar \omega / e+\hbar \omega_{m^{\prime}}\right)-I_{k k}\left(V_{o}+n^{\prime} \hbar \omega / e\right)+\right.} \\
& \left.+I_{k k}\left(V_{o}+n \hbar \omega / e\right)-I_{k k}\left(V_{o}+n \hbar \omega / e-\hbar \omega_{m^{\prime}}\right)\right]
\end{aligned}
$$

Here $I_{k k}(V)$ is the Kramers-Kronig transform of the dc I-V curve defined in Eq. 2.12 .

\subsubsection{Noise in the Quantum Theory}

Under the influence of the local oscillator, large tunneling currents flow at frequencies that are multiples $m \omega$ of the LO drive. These currents will be discussed in more detail in chapter 4 . These large currents are due to the tunneling of individual quasiparticles, so they produce shot noise at all other frequencies. Some of this noise will appear at the various signal ports, and thus will be mixed down and appear at the intermediate frequency. In this section we will outline the calculation of this noise.

The noise can be analyzed by placing a current noise source $[\mathrm{I}(\mathrm{t})-\langle\mathrm{I}(\mathrm{t})\rangle]$ in parallel with an ideal, noiseless mixer. ${ }^{1,2} \mathrm{I}(\mathrm{t})$ is the current operator for the tunnel junction, and $\langle\mathrm{I}(\mathrm{t})\rangle$ is the time-averaged current given in Eqs. 3.6 and 3.7. The difference then characterizes fluctuations about these average currents. We define the Fourier transform of the current operator as

$$
I_{T}(\omega)=\int_{-T / 2}^{T / 2} \frac{d t^{\prime}}{2 \pi} e^{i \omega t^{\prime}} I\left(t^{\prime}\right)
$$

$\mathrm{T}$ is some long time period which will become infinite at the end of the calculation. $\mathrm{I}_{\mathrm{T}}(\omega)$ has large spikes at multiples $\mathrm{m} \omega$ of the LO frequency, and a randomly fluctuating noise "floor " in between these spikes. Since the spikes do not occur at the sideband frequencies, the noise source $[\mathrm{I}(\mathrm{t})-\langle\mathrm{I}(\mathrm{t})\rangle]$ can be modeled by placing a noise source $\delta I_{m}(t)$ at each port of the mixer. The expression for the noise source is 


$$
\delta I_{m}(t)=\int_{\omega_{m}-\pi B}^{\omega_{m}+\pi B} d \omega^{\prime}\left[I_{T}\left(\omega^{\prime}\right) e^{-i \omega^{\prime} t}+I_{T}\left(-\omega^{\prime}\right) e^{i \omega^{\prime} t}\right] .
$$

$B$ is the bandwidth at the intermediate frequency over which the measurement is done. These noise generators appear at every port of the mixer and are all mixed down to the intermediate frequency. Thus we can refer all these sources to the output frequency using an effective current generator

$$
\delta I^{f f f}(t)=\int_{\omega_{m \pi B}}^{\omega_{m}+\pi B} d \omega^{\prime}\left[I_{T}^{\text {eff }}\left(\omega^{\prime}\right) e^{-i \omega^{\prime} t}+\operatorname{IT}^{\text {fff }}\left(-\omega^{\prime}\right) e^{i \omega^{\prime} t}\right]
$$

where

$$
\mathrm{ITT}_{\mathrm{T}}^{\text {eff }}\left(\omega^{\prime}\right)=\sum_{\mathrm{m}} \lambda_{0 \mathrm{~m}}^{*} \mathrm{I}_{\mathrm{T}}\left(\mathrm{m} \omega+\omega^{\prime}\right)
$$

The time-averaged mean-square noise current from this effective noise source that appears in the IF bandwidth is the output noise of the mixer and is given by

$$
\left\langle\left[\delta \mathrm{I}_{0}\right]^{2}\right\rangle=\frac{\operatorname{Lim}}{\mathrm{T} \rightarrow \infty} \frac{1}{\mathrm{~T}} \int_{-\mathrm{T} / 2}^{\mathrm{T} / 2} \operatorname{dt}\left\langle\left[\delta \mathrm{I}^{\text {eff }}(\mathrm{t})\right]^{2}\right\rangle=\mathrm{B} \frac{\operatorname{Lim}}{\mathrm{T} \rightarrow \infty} \frac{4 \pi^{2}}{\mathrm{~T}}\left\langle\left[\mathrm{ITT}^{\text {eff }}\left(\omega_{0}\right), \mathrm{IT}^{\text {eff }}\left(-\omega_{0}\right)\right]_{+}\right\rangle .
$$

We can define the current correlation matrix $\mathrm{H}_{\mathrm{mm}}$ so that,

$$
\left\langle\left[\delta \mathrm{I}_{0}\right]^{2}\right\rangle=\mathrm{B} \sum_{\mathrm{m}, \mathrm{m}^{\prime}} \lambda_{0 \mathrm{~m}} \lambda_{0 \mathrm{~m}^{\prime}} \mathrm{H}_{\mathrm{mm}^{\prime}}
$$

where

$$
\mathrm{H}_{\mathrm{mm}}=\frac{\operatorname{Lim}}{\mathrm{T} \rightarrow \infty} \frac{4 \pi^{2}}{\mathrm{~T}}\left\langle\left[\mathrm{I}_{\mathrm{T}}\left(\omega_{\mathrm{m}}\right), \mathrm{I}_{\mathrm{T}}\left(-\omega_{\mathrm{m}^{\prime}}\right)\right]_{+}\right\rangle
$$

Tucker calculated the elements of this matrix using linear response theory 1,2 and found

$$
\begin{aligned}
\mathrm{H}_{m m^{\prime}}= & \frac{\mathrm{e}}{2} \delta_{m, m^{\prime}}+\mathrm{e} \sum_{n, n^{\prime}} \mathrm{J}_{\mathrm{n}}\left(\frac{\mathrm{eV} \omega}{\hbar \omega}\right) \mathrm{J}_{\mathrm{n}^{\prime}}\left(\frac{\mathrm{eV} \omega}{\hbar \omega}\right) \delta_{m-m^{\prime}} \delta_{n-n^{\prime}} \\
& \left\{\operatorname{coth}\left[\beta\left(e V_{o}+n^{\prime} \hbar \omega+\hbar \omega_{m^{\prime}}\right) I_{d d}\left(V_{o}+n^{\prime} \hbar \omega / e+\hbar \omega_{m^{\prime}} / e\right)\right]+\right. \\
& \left.\operatorname{coth}\left[\beta\left(e V_{o}+n \hbar \omega-\hbar \omega_{m^{\prime}}\right) I_{d d}\left(V_{o}+n \hbar \omega / e-\hbar \omega_{m^{\prime}} / e\right)\right]\right\} .
\end{aligned}
$$


The first term is due to quantum fluctuations in the incident radiation field. ${ }^{24}$ The second term describes the shot noise produced by the dc current and the LO-induced currents through the tunnel junction. In order to refer this noise to the inpit, we must divide by the mixer gain, which yields an added noise spectral density of

$$
S_{m, n}=\frac{1}{4 \operatorname{Re}\left(Y_{n}\right)\left|\lambda_{0 n}\right|^{2}} \sum_{m, m^{\prime}} \lambda_{0 m} \lambda_{0 m^{\prime}}^{*} H_{m m^{\prime}} .
$$

Here $\mathrm{n}=+1$ or -1 depending on whether the mixing is done from the upper $(n=+1)$ or lower $(n=-1)$ sideband. The expression has a minimum value of $\hbar \omega$. Half of this noise can be considered to be already present as vacuum fluctuations on the incoming signal, so only $\hbar \omega / 2$ of noise is added by the mixer, in concordance with the quantum limit discussed in section 2.5 . The minimum value of Eq. 2.36 is not obvious because of the dependence of $\lambda_{0 \mathrm{~m}}$ on $\mathrm{Y}_{\mathrm{m}}$.

Extremely low noise, approaching this quantum limit, is predicted when the calculation is done with experimentally measured I-V curves of high quality SIS junctions. This low noise is mostly due to the large values of conversion gain and to the low values of sub-gap "leakage" current.

The Tucker theory of mixing predicts the high-frequency performance of a tunnel junction mixer from its dc I-V curve, its Kramers-Kronig transform, and from the values of the embedding admittance at the various frequencies involved. Much of chapter 5 is a comparison between experimentally measured and calculated mixer performance. The scheme used to deduce the hard-to-measure high-frequency admittances is also discussed there.

\section{Quantum Limit}

The accuracy of any simultaneous measurement of two conjugate variables is ultimately limited by quantum mechanics. An SIS mixer (or any phase preserving amplifier) simultaneously measures the photon number and phase of the incoming radiation, so some noise must be added in the measurement process. This is in contrast to a square-law detector, such as a bolometer, which only measures the photon number, thus to which there is no fundamental limit to its sensitivity.

Recently, Caves, using very general quantum-mechanical arguments, has shown that any narrow-bandwidth, linear, phase-preserving amplifier must add noise of spectral density referred to the input of 25

$S_{m} \geq\left|1-G_{p}{ }^{-1}\right| \hbar \omega_{s}$

where $G_{p}$ is the photon number gain. An SIS mixer in the weak signal limit and operated in the single-sideband mode is linear, preserves phase, and 
amplifies photon number. Therefore, the above limit applies. Since the IF frequency is always much less than the signal frequency, an SIS mixer operates in the regime of large photon number gain. In this regime, the quantum limit reduces to

$S_{m} \geq \hbar \omega_{s} / 2$

A mixer operated in the double sideband mode is sensitive to the relative phase of the local oscillator and the signals in the upper sideband and the lower sideband and thus this limit does not apply, and the added noise can be zero. 


\section{Chapter 3 \\ Measurement of the Quantum Susceptance \\ 3.1 Introduction}

Tunneling of quasiparticles is a quantum mechanical phenomenon. One of the consequences of such processes is that the current-voltage response is usually not instantaneous in the presence of an ac drive, provided the driving frequency is higher than the inverse of the lifetimes of the eigenstates involved. This non-instantaneous current-voltage relation consequently gives rise to a reactive component $1,2,5,19-21$ of the tunneling current in addition to a dissipative, resistive one. If the tunneling processes are elastic then the I- $\mathrm{V}$ curve contains direct information about the density of states on the two sides of the junction. In this case, the resistive (dissipative) tunneling is given by the dc I-V curve of a tunnel junction. The reactive (non-dissipative) component is related to the resistive component through a frequency Kramers-Kronig transformation, as required for any causal, linear response. ${ }^{26}$ Therefore, the high-frequency response of the junction can be completely deduced from the dc I-V curve. Consequently, the frequencydependent conductance which is associated with a nonlinear elastic tunneling I-V curve should give rise to a susceptance. The subject of this chapter is the effect of this susceptance, called quantum susceptance herein, on the small signal response of Superconductor-Insulator-Superconductor (SIS) junctions to high-frequency radiation.

It is well known that there are two types of charge carrier that tunnel across an SIS junction: Cuoper pairs and quasiparticles. They arise from the superconducting condensate and the excitations, respectively. Due to the non-instantaneous current-voltage relation, the tunneling current from each carrier contains two components in the presence of an ac drive. The inphase component is dissipative (resistive) while the out-of-phase component is nondissipative (reactive). For Cooper pair tunneling, the inphase component of the current is the Josephson $\cos \phi$ term, while the outof-phase component is the Josephson $\sin \phi$ term. $5,15,16,19-21$ For quasiparticles, the in-phase component is given by the dc quasiparticle I-V characteristic, while the out-of-phase component is the quantum susceptance or quantum reactance.1, 2, 19-21 The reactive quasiparticle tunneling current is a result of "quantum sloshing." If the energy difference of the initial and final states on two sides of the junction is different from the photon energy, no photon-assisted-tunneling can take place. Instead, the quasiparticles slosh back and forth between the two sides by absorbing and then emitting photons of the same frequency.

Werthamer derived an expression for the response function of both Cooper pairs and quasiparticles. ${ }^{5}$ The real parts of the response functions correspond to the reactive components of the tunneling currents; and the 
imaginary parts correspond to the resistive components. Using Werthamer's theory, Harris ${ }^{19-21}$ analyzed the response of an SIS junction to an RF radiation in the sinall signal limit. He correctly predicted the small effect of the quantım susceptance at zero dc bias voltage. While Josephson tunneling and quasiparticle resistive tunneling have been extensively studied, quantum susceptance has been largely ignored. This is because the contribution from the quantum susceptance to the tunneling current is only significant at frequencies high enough that the voltage associated with a quantum of the radiation, $V=\hbar \omega / e$, is larger than the voltage scale on which the $i-V$ characteristic of an SIS junction is nonlinear. ${ }^{1}$ Josephson effect devices originally showed greater promise as useful high-frequency devices, so the effects of both $\sin \phi$ and $\cos \phi$ terms on the response of Josephson junctions have been studied extensively. ${ }^{27,28}$ The quasiparticle tunneling was originally studied as a measure of the density of states for excitations. This measurement is done essentially at zero frequency so the quantum susceptance makes no contribution. This situation has changed since the invention of SIS quasiparticle direct detectors and SIS quasiparticle mixers which utilize quasiparticle tunneling for high-frequency operation. Tucker ${ }^{1}$ first studied the reactive quasiparticle tunneling at arbitrary dc and RF bias voltages. He predicted that an SIS mixer which has a non-instantaneous current-voltage relation may have a mixer gain greater than unity. In contrast, a classical resistive mixer, whose current-voltage relation is instantaneous, has a maximum mixer gain of unity. ${ }^{22}$ It was speculated that this mixer gain is due to a parametric amplification from the nonlinear quantum susceptance. However, a detailed analysis ${ }^{29}$ indicated that the effect of the quantum susceptance is quite subtle and is not directly responsible for the predicted mixer gain. It was further argued that, like the Josephson $\cos \phi$ term, ${ }^{30}$ the quantum susceptance should be difficult to detect experimentally.

In this chapter, we report experimental evidence for the quantum susceptance from a measurement of a shift of the resonant frequency of a superconducting microstrip stub resonator which contains an SIS junction. This shift of the resonant frequency is due to the change of the quantum susceptance as a function of dc bias voltage. In the following chapter we present an analysis of dc I-V curves of an SIS junction pumped with sufficient RF power that the photon-assisted-tunneling steps are clearly seen. There we demonstrate that the quantum susceptance is essential to the explanation of the negative photon-assisted-tunneling steps observed when the junction is pumped at frequencies slightly below the resonant frequency. ${ }^{8}$ This chapter is organized as follows: the theoretical background will be discussed in section 3.2, the experimental details will be described in section 3.3 , the comparison between the theory and the experiments will be discussed in section 3.4, and finally the results will be discussed in section 3.5. 


\subsection{Theoretical background}

As discussed in section 2.3, the real and imaginary parts of the quasiparticle response function are given by,

$$
\begin{aligned}
\operatorname{Re}\left[j_{\mathrm{qp}}(\omega)\right]=\mathrm{P} \int_{-}^{-} \frac{\mathrm{d} \omega^{\prime}}{\pi} \frac{\operatorname{Im}\left[\mathrm{j}_{\mathrm{qp}}\left(\omega^{\prime}\right)\right]-\hbar \omega^{\prime} / \mathrm{e} R_{\mathrm{n}}}{\omega-\omega^{\prime}} \\
=\mathrm{I}_{\mathrm{kk}}(\mathrm{V})=\mathrm{P} \int_{-\infty}^{-} \frac{\mathrm{dV^{ \prime }}}{\pi} \frac{\mathrm{I}_{\mathrm{dc}}\left(\mathrm{V}^{\prime}\right)-\mathrm{V}^{\prime} / R_{\mathrm{n}}}{\mathrm{V}^{\prime}-\mathrm{V}} .
\end{aligned}
$$

and

$$
\operatorname{Im}\left[j_{\mathrm{qp}}\left(\omega_{\mathrm{o}}\right)\right]=\mathrm{I}_{\mathrm{dc}}\left(\mathrm{V}_{\mathrm{o}}\right) .
$$

As discussed in chapter 2 , we can obtain the $\operatorname{Im}\left[j_{\mathrm{qp}}\left(\omega_{0}\right)\right]$ by simply measuring the dc I-V curve of the junction. $\operatorname{Re}\left[j_{q p}\left(\omega_{0}\right)\right]$ can then be calculated using equation 3.1.

In Fig. 3.1(a) and 3.1(b), we plot an experimentally measured I-V curve of the SIS junction studied and its voltage Kramers-Kronig transform calculated from Eq. (3.1). The peak of $I_{k k}$ at the gap voltage $V_{g}$ corresponds to the sharp nonlinearity of the dc $I-V$ curve $I_{d c}(V)$ at $V_{g}$.

Tucker ${ }^{2}$, using the above response functions, calculated the quasiparticle tunneling current in the presence of a time-dependent bias voltage, $V(t)=V_{O}+$ $\mathrm{V}_{\omega} \cos \omega t$. He found that

$$
I(t)=a_{o}+\sum_{m=1}^{\infty}\left[2 a_{m} \cos (m \omega t)+b_{m} \sin (m \omega t)\right] .
$$

The coefficients of the current at frequency $\omega$ and its harmonics are given by

$$
\begin{aligned}
& 2 a_{m}=\sum_{n=-\infty}^{\infty} J_{n}(\alpha)\left[J_{n+m}(\alpha)+J_{n-m}(\alpha)\right] I_{d c}\left(V_{o}+n \hbar \omega / e\right), \\
& 2 b_{m}=\sum_{n=-\infty}^{\infty} J_{n}(\alpha)\left[J_{n+m}(\alpha)-J_{n-m}(\alpha)\right] I_{k k}\left(V_{o}+n \hbar \omega / e\right) .
\end{aligned}
$$

Here, $I_{d c}$ and $I_{k k}$ are the same as in Eqs. (3.1) and (3.2), $J_{n}$ is the $n$th Bessel function, and $\alpha=\mathrm{eV}_{\omega} / \hbar \omega$ is the dimensionless RF voltage. Eqs.(3.3) and (3.4) indicate that many harmonics of the drive frequency $\omega$ exist in an SIS junction. The amplitudes of these current components have a nonlinear dependence on the RF drive voltage $V_{\omega}$. The non-zero value of $b_{m}$ for $m=1$ indicates that there exists an out-of-phase reactive component sin $\omega t$ as well as an in-phase component cos $\omega t$. We will show later that the current 
amplitude of the two components can be comparable. It should be noted that the dc I-V curve $I_{d c}\left(V_{0}\right)=a_{0}$ of a voltage-pumped SIS junction is completely independent of the real part of the quasiparticle response function $I_{k k}$. Therefore, $\operatorname{Re}\left(\mathrm{j}_{\mathrm{qp}}\right)$ cannot be measured from the dc I-V curves of a voltagepumped SIS junction. This is in contrast to the pair response function, whose real part $\operatorname{Re}\left(\mathrm{j}_{\mathrm{p}}\right)$ (Josephson sin $\phi$ term) contributes to a dc current at some discrete voltages which correspond to Shapiro steps. From the width of the Shapiro steps as functions of $R F$ voltage amplitude, $\operatorname{Re}\left(\mathrm{j}_{\mathrm{p}}\right)$ can be measured as a function of frequency. ${ }^{31}$

The analysis of the response of the quasiparticle tunneling current to a large amplitude $R F$ radiation is very complicated since multi-photon nonlinear processes are involved. In general, numerical computation is required and it is difficult to gain an intuitive understanding of the physics involved. However, in the small signal limit, $\alpha \ll 1$, only the one-photon process is significant, so the problem is linear. If we define an admittance $\mathrm{Y}_{\mathrm{q}}(\omega)$ as the ratio of the induced $R F$ quasiparticle current and the RF voltage, $Y_{q}(\omega)=I_{\omega} / V_{\omega}$, then from Eqs. (3.3) and (3.4) to the leading order of $\alpha$, the real and imaginary parts of $\mathrm{Y}_{\mathrm{q}}(\omega)$ are given by

$$
\begin{aligned}
\mathrm{G}_{\mathrm{q}}(\omega)=\operatorname{Re}\left[\mathrm{Y}_{\mathrm{q}}(\mathrm{w})\right]= & \frac{\mathrm{e}}{2 \hbar \omega}\left[\mathrm{I}_{\mathrm{dc}}\left(\mathrm{V}_{\mathrm{o}}+\hbar \omega / \mathrm{e}\right)-\right. \\
& \left.\mathrm{I}_{\mathrm{dc}}\left(\mathrm{V}_{\mathrm{o}}-\hbar \omega / \mathrm{e}\right)\right], \\
\mathrm{B}_{\mathrm{q}}(\omega)=\operatorname{Im}\left[\mathrm{Y}_{\mathrm{q}}(\omega)\right]= & \frac{\mathrm{e}}{2 \hbar \omega}\left[\mathrm{I}_{\mathrm{kk}}\left(\mathrm{V}_{\mathrm{o}}+\hbar \omega / \mathrm{e}\right)-2 \mathrm{I}_{\mathrm{kk}}\left(\mathrm{V}_{\mathrm{o}}\right)+\right. \\
& \left.+\mathrm{I}_{\mathrm{kk}}\left(\mathrm{V}_{\mathrm{o}}-\hbar \omega / \mathrm{e}\right)\right]
\end{aligned}
$$

$\mathrm{G}_{\mathrm{q}}$ and $\mathrm{B}_{\mathrm{q}}$ are called quantum conductance and quantum susceptance, respectively, in this thesis and in the previous papers. ${ }^{6,7}$ In the limit of low frequency, the quantum conductance $\mathrm{G}_{\mathrm{q}}(\omega)$ reduces to the classical limit $\mathrm{dI} / \mathrm{dV}$ as expected for any system whose characteristic frequency is much higher than the driving frequency. In the limit of high frequency, $\mathrm{G}_{\mathrm{q}}(\omega)$ approaches the inverse of the normal state resistance $1 / R_{n}$ at frequencies far above the gap frequency. This implies that the response of an SIS junction is like a classical diode at low frequencies and becomes Ohmic when the photon energy is much greater than the gap energy. We have shown ${ }^{6}$ that the quantum conductance $G_{q}$ and the quantum susceptance $B_{q}$ defined in Eqs. (3.5a) and (3.5b) are related through a frequency Kramers-Kronig transform, as required for any causal, linear response, ${ }^{26}$

$$
\mathrm{B}_{\mathrm{Q}}(\omega)=\mathrm{P} \int_{-\infty}^{\infty} \frac{\mathrm{d} \omega^{\prime}}{\pi} \frac{\mathrm{G}_{\mathrm{Q}^{\prime}\left(\omega^{\prime}\right)}}{\omega^{\prime}-10} .
$$


This approach is simpler than the one we discussed above. However, in this thesis, we are interested in the case of arbitrary signal strength, so we started with Eqs. (3.3) and (3.4) which apply to the general case.

Expression (3.5b) for the quantum susceptance $B_{q}$ can be interpreted geometrically. $B_{q}(\omega)$ is a measure of the curvature of the three points $I_{k k}\left(V_{0}+\right.$ $\hbar \omega / e), I_{k k}\left(V_{o}\right)$, and $I_{k k}\left(V_{o}-\hbar \omega / e\right)$. When the curvature is upward, $B_{q}$ is positive and capacitive; when the curvature is downward, $\mathrm{B}_{\mathrm{q}}$ is negative and inductive. It can be seen from Fig. 3.1(b) that as we change the dc bias voltage $V_{\mathrm{o}}$ from zero, the curvature of $I_{\mathrm{kk}}$ changes from positive to negative and back to positive. This implies that the quantum susceptance changes from capacitive to inductive and back to capacitive as shown in Fig. 3.1(d). $\mathrm{B}_{\mathrm{g}}$ has the largest capacitive value at one photon voltage $\hbar \omega /$ e below the gap voltage $\mathrm{V}_{\mathrm{g}}$ and the largest inductive value at $\mathrm{V}_{\mathrm{g}}$. In Fig. 3.1(c), we also plot the quantum conductance $G_{q}$ as a function of bias voltage. $G_{q}$ is large only within one photon voltage $\hbar \omega / \mathrm{e}$ below and above $V_{g}$, which corresponds to the voltage where a quasiparticle can tunnel to the other side by absorbing or emitting one photon.

It is easy to understand that the quantum conductance $G_{q}$ comes from the photon-assisted tunneling. It is less straightforward that the quantum susceptance $\mathrm{B}_{\mathrm{q}}$ comes frum a sloshing back and forth of quasiparticles. We will use the semiconductor model in Fig. 3.2 to help to understand both the photon-assisted tunneling and the quantum sloshing. The superconducting energy gap $2 \Delta$ splits the density of quasiparticle states into two separate bands, the conduction band and the valence band. At $T=0$, all the states in the valence band are full and all the states in the conduction band are empty. The dc bias voltage $V_{0}$ shifts the relative Fermi levels on the two sides by $\mathrm{eV}_{0}$. Consider an SIS junction in the presence of a photon field with photon energy $\hbar \omega$. Conservation of energy allows transitions to take place only between two states whose energy difference is $\hbar \omega$. Also at $T=0$, the Pauli exclusion principle requires that if one state is in the valence band then the other state must be in the conduction band.

The tunneling between states A and B in Fig. 3.2, which satisfies the condition $E_{A}+\hbar \omega=E_{B}$, is the photon-assisted tunneling ${ }^{32,33}$ which gives rise to a step-like structure on the dc I-V curve of a pumped SIS junction. This tunneling can also be assisted by absorbing more than one photon if the photon field is strong enough. The tunneling of a quasiparticle in an initial state A to final states other than B cannot occur because it violates conservation of energy. However, this does not imply that the tunneling between two such states can never take place. A quasiparticle in state A can absorb a photon $\hbar \omega$ temporarily to tunnel to a state on the right side other than state $B$, then emit a photon of the same energy and tunnel back to state A. This movement has been called "quantum sloshing" and its effect is to alter the phase of the photon field and leave the total photon number unchanged. ${ }^{1}$ Therefore, the contribution of this quantum sloshing to the 
quasiparticle tunneling current is the reactive component, which is what we called quantum susceptance.

The sign of the susceptance contributed by the quantum sloshing between two states with energies $E_{L}$ and $E_{R}$ depends on whether the energy difference $\left|E_{R}-E_{L}\right|$ is larger or smaller than the energy of the photons $\hbar \omega$ of the $R F$ drive. If $\left|E_{R}-E_{L}\right|>\hbar \omega$, then the susceptance is capacitive; if $\left|E_{R}-E_{L}\right|<$ $\hbar \omega$, the susceptance is inductive. When the energy difference between the two states is equal to the energy of the photons, the tunneling is purely resistive. These results can be understood if we model the SIS as a superposition of two-level systems.

Consider two quasiparticle states, one on the left side and the other on the right side of an SIS junction whose energy difference is $\hbar \omega_{2-1}$. The transition between these two states is analogous to the transition between two levels in an atom. Following Yariv's derivation, ${ }^{34}$ the electrical dipole moment $\mathbf{P}(\mathrm{t})$ induced by such a transition can be characterized by the "atomic" susceptibility $\chi=\chi^{\prime}-i \chi^{\prime \prime}$, such that $P(t)=\operatorname{Re}\left(\epsilon_{o} \chi E e^{i \omega t}\right)$, where $E$ is the external electrical field. The current associated with this time-varying dipole is the time derivative of the electrical dipole moment, $I(t) \propto d \mathbf{P}(t) / d t=$ $\operatorname{Re}\left(i \omega \epsilon_{0} \chi E e^{i \omega t}\right)$. Since the RF voltage $V_{\omega}$ is proportional to the electrical field

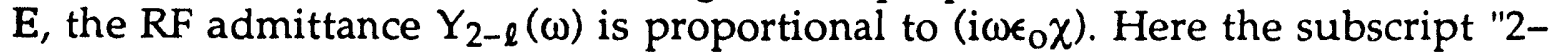
$\ell$ " is to emphasize that this admittance is the contribution only from the tunneling between these two specific states. Then from Eq. (8.1-19) in Yariv's book, we obtain the expression for the quantum conductance and susceptance which arise from these two states in the absence of inelastic scattering during the tunneling,

$$
\begin{aligned}
& \mathrm{G}_{2-\ell}(\omega) \propto \omega \chi^{\prime \prime} \propto \frac{\omega}{1+\left(\omega-\omega_{2-\ell}\right)^{2} \tau^{2}}, \\
& B_{2-\ell}(\omega) \propto \omega \chi^{\prime} \propto \frac{\omega\left(\omega_{2-\ell}-\omega\right) \tau}{1+\left(\omega-\omega_{2-\ell}\right)^{2} \tau^{2}}
\end{aligned}
$$

Here $\tau$ is the lifetime of the quasiparticle concerned. From Eq. (3.7b), at $\omega>$ $\omega_{2-\ell}, B_{2-\ell}$ is negative and the susceptance is inductive; and at $\omega<\omega_{2-\ell}, B_{2-\ell}$ is positive and the susceptance is capacitive. Finally, at $\omega=\omega_{2-\ell}, B_{2-\ell}$ is zero and the admittance is purely resistive and the conductance $\mathrm{G}_{2-\ell}$ takes a maximum value. If we assume that the quantum sloshing processes are uncorrelated, ${ }^{35}$ the total quantum conductance $G_{q}(\omega)$ and the quantum susceptance $B_{q}(\omega)$ are computed by integrating $G_{2-\ell}$ and $B_{2-\ell}$ over all the quasiparticle tunneling processes allowed by the Pauli principle. These results can also be understood qualitatively from the behavior of a classical harmonic oscillator with an intrinsic frequency $\omega_{2-\ell}$. When the drive varies slowly 
with time, $\omega<\omega_{2-\ell}$ the displacement, which is proportional to the dipole moment, follows the drive, i.e. $\mathbf{P} \propto \mathbf{E}$. When the drive varies rapidly with time, $\omega>\omega_{2-\ell}$, the displacement is $180^{\circ}$ out of phase with the drive, so $\mathrm{P} \propto-$ E.

Returning to the formal theory, we plot in Fig. 3.3 the calculated quantum conductance $\mathrm{G}_{\mathrm{q}}(\omega)$ and the quantum susceptance $\mathrm{B}_{\mathrm{q}}(\omega)$, using Eqs. (3.5) and (3.8) and the $I_{d c}$ and $I_{k k}$ in Fig. 3.1, as functions of frequency at a fixed $\mathrm{dc}$ bias voltage $\mathrm{V}_{\mathrm{o}}=2.50 \mathrm{mV}$. The peak of $\mathrm{G}_{\mathrm{q}}$ at $62 \mathrm{GHz}$ occurs when the photon energy is equal to the energy difference between the edge of the "conduction" band on one side and the edge of the "valence" band on the other side of the junction. This frequency is a simple function of dc bias voltage, $f_{o}=\left(V_{g}-V_{0}\right) / h$. Slightly above this frequency, the quantum susceptance $B_{q}$ varishes just as we expect for a two-level system. At frequencies below $f_{0}, B_{q}$ is positive and the quantum susceptance is capacitive; at frequencies above $f_{0}, B_{q}$ is negative and the quantum susceptance is inductive. The plot in Fig. 3.3 is strikingly similar to Fig. 8.2 in ref. 65, where the real and imaginary parts of the atomic susceptibility $\chi^{\prime} \propto B_{2-\ell} / \omega$ and $\chi^{\prime \prime} \propto$ $\mathrm{G}_{2-\ell} / \omega$ are plotted as functions of frequency. This strong similarity suggests that an SIS junction can be approximated as a voltage-tunable two-level system whose energy difference is $e\left(V_{g}-V_{0}\right)$. This approximation is valid because the singularities of the quasiparticle density of states at the gap energy cause a large portion of the quasiparticles to occupy the states near the gap.

Using the discussion in the last two paragraphs, we can provide a detailed physical explanation of the voltage dependence of the quantum susceptance. At $V_{0}<V_{g}-\hbar \omega / e$, the energy difference between all the states in the conduction band on one side and all the states in the valence band on the other side is greater than the photon energy, i.e. $\omega_{2-\ell}>\omega$. Therefore, $Y_{2-\ell}(\omega)$ from all possible quantum sloshing events are capacitive. As $V_{0}$ increases from zero to $V_{g}-\hbar \omega / e$, the difference $\left(\omega_{2-\ell}-\omega\right)$ becomes smaller, so the denominator in Eq. (3.7b) decreases. This results in a maximum capacitive value of the quantum susceptance $B_{q}$ at $V_{g}-\hbar \omega / e$, as show in Fig. 3.1(d). As the bias voltage $V_{0}$ increases from $V_{g}-\hbar \omega / e$, there will be states in the conduction band with energy less than $\hbar \omega$ greater than some states in the valence band on the other side. For thes pairs of states, $\omega_{2-\ell}<\omega$, so their coritribution to the quantum sloshing is inductive. This explains why the quantum susceptance $B_{q}$ becomes more inductive as $V_{o}$ increases from $V_{g}$ $\hbar \omega / e$, and has the largest inductive value at the gap voltage $V_{g}$, as shown in Fig. 3.1(d).

Although the above discussion was carried out at $\mathrm{T}=0$ for simplicity, the results are still valid at finite temperature. Two modifications should be introduced in the above discussion at finite temperatures. First, the superconducting energy gap is reduced. Second, the states in the valence band are not completely filled, the occupation probability is given by the Fermi distribution $f(E)$. Similarly, the states in the conduction band are not 
completely empty, and the unoccupied probability is given by $1-f(E)$. These two modifications at finite temperature affect the dc I-V curve in the same way as they affect the high-frequency response of the SIS junction. Therefore, the RF admittance of an SIS junction is still given by Eqs. (3.8a) and (3.8b) as long as its dc $I-V$ curve at $T \neq 0$ is still due to elastic tunneling. ${ }^{19-21}$

In the general case, $\alpha=\mathrm{eV}_{\omega} / \hbar \omega$ can be any value and vre must consider a complicated nonlinear solution of Eq. (3.4) to analyze the response of an SIS junction to $R F$ radiation. We can still define an admittance $Y(\omega)=I_{\omega} / V_{\omega}$, where $I_{\omega}$ and $V_{\omega}$ are the current and voltage at frequency $\omega$. In this case, $Y(\omega)$ will be a function of $V_{\omega}$ as well as a function of $V_{0}$ and $\omega$. Numerical computation is required for detailed analysis. However, some of the qualitative features discussed above in the linear limit will still apply as long as $\alpha$ is not so much greater than unity that multi-photon processes dominate the one-photon process. ${ }^{8}$ One of the important features is that the quantum susceptance takes its maximum capacitive value at one photon voltage below the gap $V_{g}-\hbar \omega / e$, and changes to an inductive value as the bias voltage increases to the gap voltage $\mathrm{V}_{\mathrm{g}}$. We will show later in chapter 4 that this feature is responsible for the photon-assisted-tunneling steps with negative dynamic resistance which were observed when the embedding admittance is slightly inductive.

\subsection{Experimental details}

As discussed in section 3.2, the reactive part of the quasiparticle response function (or, equivalently, the quantum susceptance $B_{q}$ ) has no contribution to the tunneling current when the bias voltage is purely dc, i.e. $V(t)=V_{0}$. Also, the quantum susceptance $B_{q}$ has no effect on the dc I-V curve of an SIS junction pumped by an RF voltage source whose amplitude $V_{\omega}$ is independent of $\mathrm{dc}$ bias voltage. Consequently, the quantum susceptance cannot be measured in a dc voltage biased SIS junction, or from the dc I-V curves of an RF voltage biased SIS junction.

The most straightforward and convenient way to measure a reactive ei ement is to measure the resonant frequency of a resonator which contains th e element to be measured. In a less direct way, the quantum susceptance $B_{q}$ Cin be measured from the shape of the I-V curves of an SIS junction pumped by an RF source with a non-zero output impedance. The first method gives a direct and definitive measurement of the quantum susceptance. The second method gives an independent check and can also help in understanding the role of the quantum susceptance in the RF impedance match, especially in the large signal limit. This impedance match is crucial for many SIS devices, such as SIS direct detectors, ${ }^{36}$ SIS heterodyne mixers, ${ }^{2}$ and SIS parametric amplifiers. ${ }^{37}$ In this chapter we focus on the first method of measurement.

We have constructed a millimeter wave resonant circuit by using a superconducting microstrip stub and an SIS junction. This resonator is quasioptically coupled to the radiation source by a planar antenna and several 
lenses. ${ }^{11} \mathrm{~A}$ photograph and a schematic drawing of the junction and microstrip stub located at the center of a log-periodic antenna are shown in Figs. 3.4(a) and 3.4(b). The response of this resonator to an RF signal can be analyzed using the equivalent circuit shown in Fig. 3.4(c). The signal and the antenna are represented by an RF current source in parallel with its source admittance $Y_{A}$. The SIS junction is represented by the parallel combination of the quantum conductance $G_{q}(\omega)$, quantum susceptance $B_{q}(\omega)$, and the geometric capacitance $C$. The admittance of the superconducting microstrip stub is essentially reactive and can be represented by a susceptance $B_{\text {stub }}(\omega)$. The loss of the stub at the RF frequency can be modeled by a conductance in parallel with $B_{\text {stub }}(\omega)$. This loss does not affect the value of the susceptance $B_{\text {stub }}(\omega)$ to first order, and therefore it is unimportant in the determination of the resonant frequency.

In order to measure the quantum susceptance $B_{q}$, we need to know the imbedding susceptance $B_{e m b}$, which is the total susceptance that is independent of dc bias voltage. In the equivalent circuit in Fig. 3.4(c), the imbedding susceptance $B_{e m b}$ is the sum of the susceptances of the junction capacitance $\omega C$, and of the microstrip stub $B_{\text {stub }}(\omega)$, and of the antenna $\operatorname{Im}\left(Y_{A}\right)$. The resonance of the equivalent circuit of Fig. 3.4(c) corresponds to the condition $B_{\text {total }}=B_{q}(\omega)+B_{e m b}(\omega)=0$. Without the quantum susceptance $B_{q}$, the resonant frequency would be independert of bias voltage. However, since $B_{q}$ changes rapidly with dc bias voltage $V_{0}$ as shown in Fir. 3.1(d), we expect that the resonant frequency will change as $V_{O}$ changes.

The susceptance of the capacitance is simply $\omega C$, and the susceptance of the stub $B_{\text {stub }}(\omega)$ can be calculated using formulas in a standard microwave engineering text book. ${ }^{38}$ The expression of the susceptance of an antenna can be quite complicated in general. However, for a special class of planar antennas called "self-complementary antennas", in which the pattern of the metallic part is the same as that of the dielectric part, the admittance of the antenna is real and independent of frequency. ${ }^{39}$ The antenna admittance is given by $Y_{A}=\left(1+\epsilon_{r}\right)^{1 / 2} 3.74 \times 10^{-3} \Omega^{-1}$, where $\epsilon_{\mathrm{r}}$ is the relative dielectric constant of the substrate. Use of a self-complementary antenna greatly simplifies the characterization of the embedding admittance. In this experiment, we have used a circular-toothed log-periodic antenna which has been measured to have a high antenna efficiency $(\sim 60 \%)$ and a nearly Gaussian antenna beam pattern. ${ }^{39,40}$ As shown in Fig. 3.4(a), the antenna is self-complementary. We have used a fused quartz substrate, which has a relative dielectric constant $\epsilon_{\mathrm{r}}=3.85$ at millimeter wave frequencies. ${ }^{41,42}$ This gives an antenna admittance of $Y_{A}=8.3 \times 10^{-3} \mathrm{~W}^{-1}$.

We have used a superconducting microstrip stub with the stub made out of $\mathrm{Pb}-\mathrm{In}-\mathrm{Au}$ alloy and the ground plane of $\mathrm{Nb}$. As shown in Figs. 3.4(a) and 3.4(b), the stub contains two sections, a narrow section 1 and a wide section 2 . The widths and the lengths of the two sections are: $\mathrm{w}_{1}=6 \mu \mathrm{m}, \mathrm{w}_{2}=$ 
$40 \mu \mathrm{m}, \ell_{1}=135 \mu \mathrm{m}$, and $\ell_{2}=260 \mu \mathrm{m}$. The phase velocity within the microstrip line is $\mathrm{v}=1 /\left(\mathrm{L}_{\mathrm{s}} \mathrm{C}_{\mathrm{s}}\right)^{1 / 2}$, where $\mathrm{L}_{\mathrm{s}}=\left(\mu_{\mathrm{o}} / \mathrm{k} \omega\right)\left[\mathrm{t}+\lambda_{1} \operatorname{coth}\left(\mathrm{t}_{1} / \lambda_{1}\right)+\right.$ $\left.\lambda_{2} \operatorname{coth}\left(t_{2} / \lambda_{2}\right)\right]$ is the inductance per unit length, 74 and $C_{s}=k \epsilon_{r} \epsilon_{o} W / t$ is the capacitance per unit length. ${ }^{43} \mathrm{t}$ and $\epsilon_{\mathrm{r}}$ are the thickness and the dielectric constant of the insulating layer ( $\mathrm{SiO}$ in our case), $\mathrm{t}_{1,2}$ and $\lambda_{1,2}$ are the thicknesses and the London penetration depths of the ground $(\mathrm{Nb})$ and top $(\mathrm{Pb}-\mathrm{In}-\mathrm{Au})$ plane, and $\mathrm{k}$ is a fringing factor close to unity. Using the designed values, $\epsilon_{\mathrm{r}}=5.7,{ }^{44} \mathrm{t}=3000 \AA, \mathrm{t}_{1}=2000 \AA, \mathrm{t}_{2}=4250 \AA, \lambda_{\mathrm{Nb}}=850 \AA,{ }^{44} \lambda_{\mathrm{Pb}-\mathrm{In}-\mathrm{Au}}=$ $1450 \AA,{ }^{44}$ the phase velocity is $v=0.30 \pm 0.01 \mathrm{c}$. The length of the wider section is $1 / 4$ of the wavelength at $87 \mathrm{GHz}$, so the wider section transforms an $\mathrm{RF}$ open circuit at point $A$ to an RF short circuit at point $B$ in Fig. 3.4 (b). ${ }^{38}$ This two-section stub has a slower variation of the susceptance as a function of frequency than an one-section open-ended stub, so the effect of the quantum susceptance is more profound. ${ }^{45}$ The length of the narrow section is $1 / 8$ of the wavelength at $85 \mathrm{GHz}$ which transforms the RF short to an inductive admittance. The total susceptance of the two-section stub is given by ${ }^{38}$

$$
B_{\text {stub }}(\omega)=\frac{Y_{1}\left[Y_{2} \tan \left(\beta \ell_{2}\right)+Y_{1} \tan \left(\beta \ell_{1}\right)\right]}{Y_{1}-Y_{2} \tan \left(\beta \ell_{1}\right) \tan \left(\beta \ell_{2}\right)} .
$$

Where $\beta=\omega / v, Y_{1,2}=\left(C_{s 1,2} / L_{s 1,2}\right)^{1 / 2}$ are the characteristic admittances of section 1 (narrow) and section 2 (wide) of the stub, $Y_{1}=0.124 \Omega^{-1}$, and $Y_{2}=$ $0.637 \Omega^{-1}$. We have shown that the expression of the susceptance of the stub $B_{\text {stub }}(\omega)$ remains the same when there is a small RF loss in the stub. ${ }^{46}$

In order to measure the small-signal frequency response of the junction/stub resonator, the RF power coupled to the resonator must be less than $10 \mathrm{pW}$ so for $\mathrm{G}_{\mathrm{q}}=0.01 \Omega^{-1} \mathrm{a}=\mathrm{eV}_{\omega} / \hbar \omega \ll 1$ at $75 \mathrm{GHz}$ and Eq. (3.8) applies. Consequently, we need a very sensitive detector. Also, the frequency dependence of the detector must be known in order to separate the frequency response of the resonator from that of the detector. We have used the internal detection mechanism in the SIS junction to measure the frequency response of the resonator. SIS direct detectors are known to be among the most sensitive $4.2 \mathrm{~K}$ video detectors at millimeter wave frequencies, ${ }^{47}$ and they have been proved to be very useful in measuring the frequency response of millimeter and submillimeter wave resonators. ${ }^{46}$ The frequencydependent responsivity of the SIS direct detector can be easily calculated from Tucker's theory. ${ }^{1}$ There is also a major advantage of this scheme: because of the proximity of the SIS detector to the resonator, there is no Fabry-Perot interference between them. The output of the SiS detector as a function of RF frequency is the prosiuct of the frequency response of the resonator, the spectrum of the source, and the frequency-dependent responsivity of the SIS detector. 
The current responsivity $S_{I}$ of an SIS direct detector, defined as the induced dc current per unit RF power absorbed, as a function of frequency is given by, 1

$$
\mathrm{S}_{\mathrm{I}}(\omega)=\frac{\Delta \mathrm{I}_{\mathrm{dc}}}{\mathrm{P}_{\omega}}=\frac{\mathrm{e}}{\hbar \omega} \frac{\mathrm{I}_{\mathrm{dc}}\left(\mathrm{V}_{\mathrm{o}}+\hbar \omega / \mathrm{e}\right)-2 \mathrm{I}_{\mathrm{dc}}(\mathrm{V})+\mathrm{I}_{\mathrm{dc}}\left(\mathrm{V}_{\mathrm{o}}-\hbar \omega / \mathrm{e}\right)}{\mathrm{I}_{\mathrm{dc}}\left(\mathrm{V}_{\mathrm{o}}+\hbar \omega / \mathrm{e}\right)-\mathrm{I}_{\mathrm{dc}}\left(\mathrm{V}_{\mathrm{o}}-\hbar \omega / \mathrm{e}\right)}
$$

Here $P_{\omega}=\operatorname{Re}\left(I_{\omega} V_{\omega}{ }^{*} / 2\right)$ is the $R F$ power actually dissipated in the SIS junction. Note the absence of the reactive quasiparticle response function $I_{k k}$ in Eq. (3.12), which implies that the quantum susceptance $B_{q}$ does not affect the responsivity. As pcinted out by Tucker, ${ }^{2} \mathrm{~S}_{\mathrm{I}}(\omega)$ reduces to a frequencyindependent classical current responsivity $\left(\mathrm{d}^{2} \mathrm{I} / \mathrm{dV}^{2}\right) / 2(\mathrm{dI} / \mathrm{dV})$ at low frequencies; and approaches a quantum limit e/ $\hbar \omega$ at frequencies so high that the voltage associated with one photon $\hbar \omega / e$ is larger than the width of the current rise at the sum gap voltage. The induced dc current per unit available $R F$ power $P_{A}$ in the SIS junction as a function of RF frequency is then given by

$$
\frac{\Delta I_{d c}}{P_{A}}=S_{I}(\omega)\left[1-\left|\frac{Y_{A}-Y_{j}^{*}}{Y_{A}+Y_{j}}\right|^{2}\right],
$$

where $Y_{J}=G_{q}+i\left(B_{q}+\omega C+B_{s t u b}\right)$ is the total admittance of the SIS junction and the stub, and $\mathrm{S}_{\mathrm{I}}(\omega)$ is the current responsivity defined in Eq. (3.9). The second factor on the right hand side of Eq. (3.10) is the RF coupling coefficient $C_{R F}$ defined in previous publications. ${ }^{11} C_{R F}$ is the fraction of the available $R F$ power which is delivered to the dissipative element $\mathrm{G}_{\mathrm{q}}$. Eq. (3.10) implies that the induced dc current is the product of the RF coupling coefficient $C_{R F}(\omega)$ and the current responsivity $\mathrm{S}_{\mathrm{I}}(\omega)$. Since $\mathrm{S}_{\mathrm{I}}(\omega)$ is a smooth function of frequency except at $e\left(V_{g}-V_{0}\right) / \hbar$, the frequency dependence of the RFinduced dc current $\Delta \mathrm{I}_{\mathrm{dc}}$ is mainly determined by the frequency dependence of $\mathrm{C}_{\mathrm{RF}}(\omega)$. Therefore, the frequency which corresponds to the maximum $\Delta \mathrm{I}_{\mathrm{dc}}$ is mainly determined by the resonance condition of the resonator, that is, $\operatorname{Im}\left(Y_{j}\right)$ $=B_{q}+\omega C+B_{\text {stub }}=0$. When this condition is met, the RF coupling coefficient $\mathrm{C}_{\mathrm{RF}}$ has the maximum value.

We also need to know the power spectrum of the RF source. We have used both a tunable coherent millimeter wave source which utilizes the Gunn effect ${ }^{48}$ and an incoherent source from the output of a Fourier transform spectrometer (FTS). Calibration of the coherent power incident upon the resonator was difficult due to Fabry-Perot resonance within the source. These resonances have sharper peaks than that of the stub/junction resonator so they dominate the measured response. The short coherence length of the radiation from the FTS eliminates most of this problem. In this 
paper, the resonan' frequencies and the widths of the resonances of the stub/junction resonator were measured using the FTS. The coherent source was used to study the shape of the photon-assisted-tunneling I-V curves.

The FTS used in this experiment is a far-infrared Michelson interferometer ${ }^{49}$ operated in the step-and-integrate mode. The output spectrum of the FTS is the blackbody radiation from a Hg-arc lamp at $500 \mathrm{C}^{\circ}$, modified by the efficiency of a $250 \mathrm{~mm}$ thick Mylar beamsplitter. Since the antenna-coupled SIS direct detector is sensitive to only a single electromagnetic mode, and the source is in the R.ayleigh-Jeans limit, the power spectrum of the source is given by a constant multiplied by the beamsplitter efficiency $\eta_{b m}$, which is a smooth function of frequency. ${ }^{46}$ For $250 \mathrm{~mm}$ thick Mylar film at $45^{\circ}$ to the beam with a relative dielectric constant $\epsilon_{\mathrm{r}}=3$, the beamsplitter efficiency $\eta_{\mathrm{bm}}$ is slowly increasing with frequency in the frequency range of interest. 46

The experimental apparatus used in this work is essentially the same as was used in our quasioptical SIS mixer experiments to be discussed in detail in chapter 6 . The output of the FTS is connected to the cryostat through a 1meter long, $11-\mathrm{mm}$ diameter light pipe. The cryostat has a $25-\mathrm{mm}$ diameter window which is covered with a $25-\mathrm{mm}$ thick polypropylene window, which transmits almost 100 per cent at millimeter wave frequencies. Within the cryostat, the signal beam is focused by a $\mathrm{f} / 0.85 \mathrm{TPX}$ lens, and then further focused by a hyperhemispherical quartz lens to a $f / 0.5$ converging beam whose beam waist occurs at the flat side of the hyperhemispherical quartz lens, where the log-periodic antenna with the junction and the resonator is centered. The quartz lens is heat sunk to the liquid helium tank through a copper support. The temperature of the SIS junction is estimated to be $4.5 \mathrm{~K}$ for an unpumped helium bath. Under unpumped condition, the liquid helium in the cooling tank can last about 10 hours as compared to $\sim 5$ hours when the helium is pumped. The longer hold time allows us to inprove the signal/noise ratio by using longer integration times. Therefore, all the results reported in this chapter were obtained at $4.2 \mathrm{~K}$ bath temperature. This temperature is cold enough for our experiment since our all-Nb SIS junctions have a relatively high $\mathrm{T}_{\mathrm{c}}(\sim 9 \mathrm{~K})$ so the operating temperature is about half of the transition temperature.

The SIS junction used in this experiment was fabricated at the National Institute of Standards and Technology at Boulder. It is a $\mathrm{Nb}^{\prime} \mathrm{Al}_{2} \mathrm{O}_{3} / \mathrm{Nb}$ sandwich made using the tri-layer process. ${ }^{50}$ The critical current density of the SIS junction is about $500 \mathrm{~A} / \mathrm{cm}^{2}$. The normal resistance of $70 \Omega$ is approximately matched to the antenna impedance. The I- $\mathrm{V}$ curve of the junction shows a low leakage current and a sharp gap structure even at $4.5 \mathrm{~K}$, as shown in Fig. 3.1(a). The sharp gap structure causes a dramatic peak in $\mathrm{I}_{\mathrm{kk}}(\mathrm{V})$ at the gap voltage $\mathrm{V}_{\mathrm{g}}$. This peak, and the associated large values of curvature, are essential to observe the effects of the quantum susceptance as 
discussed above. The junction has been thermally cycled between room temperature and liquid helium temperature over 30 times, and the I-V characteristic has not changed. The junction area is estimated to be $2.5 \times 2.5$ $\mu \mathrm{m}^{2}$, which gives a geometric capacitance of $0.28 \pm 0.03 \mathrm{pF}$ if we assume a specific capacitance value of $45 \pm 5 \mathrm{fF} / \mathrm{mm}^{2}{ }^{21}$ This capacitance value gives a susceptance of $0.14 \Omega^{-1}$ at $80 \mathrm{GHz}$. Fig. 3.1(d) indicates that the change of the quantum susceptance is as large as $0.05 \Omega^{-1}$ between 2.4 and $2.7 \mathrm{mV}$, which is significant compared to that of the junction capacitance. Therefore, the change of the quantum susceptance as a function of dc bias voltage should have a very noticeable effect on the resonant frequency of the stub/junction resonator.

\subsection{Data Analysis}

In this chapter, we present the measured resonant frequency and the width of the resonance peaks as functions of dc bias voltage. These data were obtained from spectra measured in the small signal limit using a Fourier transform spectrometer. The effects of the quantum susceptance were easily measurable from these data. In chapter 4 , we will present the dc I-V characteristics for junctions exposed to large amplitude narrow-band millimeter-wave radiation. Analysis of those data in the large signal limit provides an additional, though less direct measurement of the quantum susceptance. After these measurements were made, additional measurements were performed at Yale University ${ }^{52}$ using a millimeterwave reflectometer which provided further evidence for the quantum susceptance. The Yale measurements were done both in the small and large signal limits.

The interferograms in this experiment were obtained from the RFinduced dc current $\Delta \mathrm{I}_{\mathrm{dc}}$ as defined in Eq. (3.10) as a function of the difference between the two optical paths of the FTS. These interferograms were measured in the step-and-integrate mode, with the integration time typically $\sim 1.5$ seconds. The spectra were obtained by Fourier transformation of the product of the interferogram and the apodization function. ${ }^{49}$ We chose to use an apodization function with a form of $\left[1+\cos \left(x \pi / x_{\max }\right)\right] / 2$, where $x$ is the path length difference and $x_{\max }$ is the maximum of the path length difference used in the experiment. This apodization function lowers side peaks of the instrument function at the expense of a moderate increase of the width of the resonance peak. Figs. 3.5(a) and 3.5(b) show interferograms measured at two bias voltages, $\mathrm{V}_{\mathrm{o}}=2.350 \mathrm{mV}$, and $\mathrm{V}_{\mathrm{o}}=2.500 \mathrm{mV}$. At $\mathrm{V}_{\mathrm{o}}=$ $2.350 \mathrm{mV}$, the value of the quantum conductance $\mathrm{G}_{\mathrm{q}}$ is low as shown in Fig. 3.1(c), so the Q-value of the stub/junction resonator is high and the peak of the resonance is narrow. Consequently, the fringe amplitude decreases slowly as the path difference increases as shown in the interferogram in Fig. 3.5(a). At $V_{o}=2.500 \mathrm{mV}$, the value of the quantum conductance $\mathrm{G}_{\mathrm{q}}$ is high due to the onset of the photon-assisted-tunneling, so the $\mathrm{Q}$-value of the 
stub/junction resonator is low and the peak of the resonance is broader than that measured at $V_{0}=2.350 \mathrm{mV}$. Consequently, the fringe visibility in the interferogram decreases rapidly as the path difference increases as shown in Fig. 3.5(b). The corresponding spectrum shown in Fig. 3.5(d) shows a broader peak than that in Fig. 3.5(c). Besides the apparent difference in the widths of the resonances in the two spectra, the frequencies which correspond to the peaks of the two spectra differ by a noticeable amount.

In order to improve the signal/noise ratio of the measured spectra, we co-added 5-10 spectra measured at a given bias voltage. After normalizing these spectra to the beamsplitter efficiency $\eta_{b m}$, we obtain the resonant frequencies by least-mean-square fitting the top $50 \%$ part of the resonance peaks with 2 nd to 4 th order polynomials. The degree of the polynomials in the fitting is determined by the asymmetry of the peak. The error bars on the measured resonant frequencies are chosen as the frequency ranges in whichthe fitting polynomials are over $90 \%$ of their peak values. The result is plotted in Fig. 3.6(a) as a function of dc bias voltage $V_{0}$. Below $2.150 \mathrm{mV}$ and above $2.650 \mathrm{mV}$, the signal/noise ratio of the spectra is very poor due to the roll-off of the current responsivity $S_{I}$ of the SIS direct detector. Therefore, no data are plotted outside of this range. The error bars are twice as large for $V_{0}>$ $2.450 \mathrm{mV}$ as those for $<2.450 \mathrm{mV}$ because the peaks are broader for $V_{0}>2.450$ $\mathrm{mV}$ due to the sharp increase of the quantum conductance $\mathrm{G}_{\mathrm{q}}$. Fabry-Perot fringes appear on these broad peaks if we keep the resolution of the FTS the same as for the narrow peaks. These Fabry-Perot fringes probably arise from the standing waves between the SIS junction and the TPX lens. In order to average over those Fabry-Perot fringes, we have used a lower resolution of $0.3175 \mathrm{~cm}^{-1}$ in our FTS which resulted in large error bars for the measured resonant frequencies above $2.450 \mathrm{mV}$. The experimentally measured resonant frequencies clearly show a smooth shift as the dc bias voltage changes. The most dramatic change of the resonant frequency takes place within the voltage range from $2.400 \mathrm{mV}$ to $2.650 \mathrm{mV}$, where it changes " om $73 \mathrm{GHz}$ to 87 $\mathrm{GHz}$. From Fig. 3.1(d), we can see that the quantum susceptance $\mathrm{B}_{\mathrm{q}}$ changes rapidly from capacitive to inductive in exactly the same voltage range.

In order to make accurate comfarisons between theory and experiment, we obtain the theoretically calculatec resonant frequencies using the same method used to obtain the experimental resonant frequencies. First, we compute the RF-induced dc current as a function of RF frequency using Eq. (3.10). Second, we convolve these computed spectra with the Fourier transform of the apodization function which was used in the Fourier transformation of the experimental interferograms. ${ }^{49}$ Third, we chose the same number of computed data points at the same discrete frequencies as we did from the experimental data. Finally, for each spectrum, we fit these discrete computed points with a polynomial with the same degree as was used in fitting the experimental data. The theoretically calculated curve for the resonant frequency as a function of $V_{0}$ is shown in Fig. 3.6(a) as the solid 
line, and it is in excellent agreement with the experimental results. We would like to emphasize that the values of two key parameters, the junction capacitance $C=0.275 \mathrm{pF}$, and the phase velocity $\mathrm{v}=0.286 \mathrm{c}$, which vere used in our theoretical computation, are essentially the same as the ones we estimate from the geometric dimensions, $0.28 \pm 0.03 \mathrm{pF}$ and $0.30 \pm 0.01 \mathrm{c}$. As a comparison, the dashed line, which is essentially flat and obviously differs from the experimental results, is the theoretically calculated resonant frequency as a function of $\mathrm{V}_{\mathrm{o}}$ without including the quantum susceptance $\mathrm{B}_{\mathrm{q}}$. The weak voltage dependence of the dashed line is due to the change of the current responsivity $S_{I}(\omega)$ with $V_{0}$. Clearly, these results provide decisive evidence for the quantum susceptance.

We have also investigated the effect of Josephson oscillation on the shift of the resonant frequency by applying a magnetic field to change the Josephson critical current. From Eqs. (3.1) and (3.2), we can see that the pair tunneling current also contains a reactive component, the sin $\phi$ term. This reactive component from the pair tunneling may also affect the resonant frequency of the stub/junction resonator. If there is any significant effect from the pair tunneling, then this effect should be changed as we modulate the Josephson critical current with a magnetic field. We did not measure any change of the resonant frequency within cur experimental accuracy up to a field corresponding to several quanta of magnetic flux in the SIS junction. This is probably because, at bias voltages from 2.15 to $2.65 \mathrm{mV}$, the Josephson current oscillates at frequencies above $1 \mathrm{THz}$, which is strongly shunted by the junction capacitance.

We discovered a strong signal at the output of the SIS detector at $\mathrm{V}_{\mathrm{O}}=$ $0.158 \mathrm{mV}$, which corresponds to a $77 \mathrm{GHz}$ Josephson oscillation. The level of this strong signal is comparable to the largest signal obtained in the voltage range from $2.100 \mathrm{mV}$ to $2.650 \mathrm{mV}$ using quasiparticle direct detection. This detection is a result of a Josephson homodyne detection in a self-pumped mode. In this mode, the Josephson current, which oscillates at $\omega_{j} / 2 \pi=2 \mathrm{eV}_{\mathrm{o}} / \mathrm{h}$ $=77 \mathrm{GHz}$, which coincides with the resonant frequency of the microstrip stub resonator, mixes with the RF signal at the same frequency and produces a dc output. We found that the signal level at the output of the detector is a very sensitive function of the dc bias voltage. At voltages below $0.150 \mathrm{mV}$ and above $0.170 \mathrm{mV}$, the signal level decreases to essentially the level of the broadband noise. A similar detection mode was reported by Richards and Sterling ${ }^{53}$, in which the Josephson detector exhibited a very narrow frequency response at the resonant frequency of a cavity. The interferogram obtained in this detection mode is very similar to those obtained using quasiparticle direct detection. The peak frequency of the resonance is the same as the Josephson oscillation frequency, $77 \mathrm{GHz}$. We would like to point out that at this low bias voltage, the curvature of $\mathrm{I}_{\mathrm{kk}}(\mathrm{V})$ is almost zero, as can be seen from Fig. 3.1(b). So the quantum susceptance is negligible compared to that of the embedding structures. In addition, the susceptance of the Josephson sin $\phi$ term is 
negligible at this low RF power level. ${ }^{27}$ Therefore, the measured resonant frequency should be the resonant frequency of the microstrip stub and the junction capacitance. The coincidence of this measured resonant frequency and the calculated one without including the quantum susceptance (dashed line in Fig. 3.6(a)) is an additional verification of the values of the junction capacitance $C$ and the phase velocity $v$ which are used in our calculations.

In Fig. 3.6(b), we plot the 3-dB linewidths $\Delta f$ of the resonance peaks as a function of the dc bias voltage. The experimental value of $\Delta f$ were obtained from the best fitted polynomials. The solid line is calculated using the same apodization function used in the experiment. Again, the agreement between experiment and theory is excellent. This comparison provides an additional verification of the values of $C$ and $v$ in our calculations. The sharp increase of $\Delta \mathrm{f}$ at $2.450 \mathrm{mV}$ corresponds to the sharp increase of the quantum conductance $\mathrm{G}_{\mathrm{q}}$ at one photon voltage $\hbar \omega / \mathrm{e}$ below the gap voltage $\mathrm{V}_{\mathrm{g}}$. Note from Fig. 3.1(d) that the quantum susceptance has the largest capacitive value at this voltage, $\mathrm{V}_{\mathrm{g}}-\hbar \omega / \mathrm{e}$, so the resonant frequency is the lowest as shown in Fig. 3.6(a). There is some disagreement between the theoretical and experimental values of $\Delta f$ at $V_{o}=2.45 \mathrm{mV}$. This discrepancy arises because the quantum conductance $G_{q}$ depends on the $I-V$ curve around $V_{0}+\hbar \omega / e$ which, at $V_{0}=$ $2.45 \mathrm{mV}$, lies just above the sum gap voltage. Our junction exhibits a negative resistance in this region due to the proximity effect ${ }^{54}$. This is not correctly measured by our I-V curve measurement system.

\subsection{Discussion}

Quantum mechanical tunneling usually resuits in a noninstantaneous current-voltage relation if the time scale of the modulation is shorter than the lifetime of the quasiparticles involved. This noninstantaneous current-voltage relation will consequently give rise to a nondissipative reactive component as well as a dissipative, resistive component in the tunneling current. Such a reactive component, which is called the quantum susceptance, should exist in many types of tunneling devices. In a special case in which the tunneling is elastic so the quasiparticles emitted from one side of a junction reach the other side at the same energy level, the high-frequency response function can be simply measured from the dc I-V curve. SIS tunnel junctions with high quality tunnel barriers is an example. Other devices, such as quantum well resonant tunneling devices in which electrons tunnel through a double barrier quantum well, may also exhibit similar behavior.

The effect of the quantum susceptance is usually complicated at low frequencies because the experimentally acceptable signal/noise ratio requires that the dimensionless $R F$ voltage $a=e_{\omega} / \hbar \omega>>1$. In this limit multi-photon processes dominate so the system is highly nonlinear. In a linear scheme in which $\alpha<<1$, the effect of the quantum susceptance can be predicted analytically. However, $\alpha<<1$ requires sufficiently high frequency so $V_{\omega}$ is 
large enough for an acceptable signal/noise ratio. We have obtained definitive experimental evidence for the existence of the quantum susceptance by studying the response of an SIS junction to a weak RF radiation at millimeter wavelengths. We have measured the shift of the resonant frequency of a resonator which contains an SIS junction. The observed $19 \%$ shift, from $73 \mathrm{GHz}$ to $87 \mathrm{GHz}$ as the dc bias voltage is changed from $2.40 \mathrm{mV}$ to $2.65 \mathrm{mV}$, is due to the change of the quantum susceptance with bias voltage. This is in excellent agreement with Werthamer-Tucker theory and is a direct experimental evidence of the existence of the quantum susceptance. Our result has therefore, for the first time, directly verified one of the important aspects of this theory. 


\section{Chapter 4 \\ Analysis of Pumped I-V Curves}

\subsection{Introduction}

When millimeter wave radiation is incident on an SIS tunnel junction, a series of steps is observed on the dc I-V curve above and below the current rise at the sum-gap. These steps correspond to the stimulated emission or absorption of one or more photons during the tunneling process. The I-V curve with radiation applied is commonly called the pumped I-V curve. This chapter gives a detailed discussion of the influence of the source admittance as seen by the junction (the embedding admittance) on the exact shape of the pumped I-V curve. First we discuss the case when the junction is pumped by a voltage source. This case is simple to analyze but experimentally unrealistic. We then discuss the influence of the source admittance on the magnitude of the pump voltage. Borrowing from the discussion of junction admittance from the last chapter, we find that pump voltage is dependent on the dc bias voltage. This causes the pumped I-V curve to differ from that calculated using a RF voltage source. We then show accurate fits to experimentally measured pumped I-V curves obtained by using the embedding admittance as a free parameter. We then discuss this theoretical fitting to the pumped I-V curve as a method of measuring the embedding admittance under experimental conditions.

\subsection{Photon Assisted Tunneling}

The dc quasiparticle tunneling current pumped by a time-dependent potential $V(t)=V_{\omega} \cos (\omega t)$ is given by ${ }^{1,33}$

$$
\mathrm{I}\left(\mathrm{V}_{0}, \mathrm{~V}_{\omega}\right)=\sum_{n=-\infty}^{\infty} \mathrm{J}_{n}^{2}(\alpha) \mathrm{I}_{\mathrm{dc}}\left(\mathrm{V}_{\mathrm{o}}+\mathrm{n} \hbar \omega / \mathrm{e}\right)
$$

where $\alpha=\mathrm{eV}_{\omega} / \hbar \omega, \mathrm{J}_{\mathrm{n}}(\mathrm{a})$ is the nth order Bessel function of the first kind, and $\mathrm{I}_{\mathrm{dc}}(\mathrm{V})$ is the dc current that flows through the unpumped junction at dc bias voltage $V$. This expression assumes that all higher harmonics of the ac waveform are shorted. We assume that this is the case throughout this chapter. This assumption is identical to the 3-port approximation used to evaluate mixer performance in later chapters. The approximation is valid for all experimental results reported in this thesis due to the relatively large geometrical capacitance of the junctions used in our work.

If the junction is pumped by an rf voltage source, calculation of the pumped dc I-V curve can be accomplished by evaluating this expression numerically at each dc bias voltage. The result of this calculation is shown in Fig. 4.1 for a typical I-V curve. Note that this is different that the classical method of calculating the pumped I-V curve in which the time-dependent 
voltage is applied to the dc I-V curve and the time-averaged current is calculated. The classical analysis would not produce the steps seen in Fig. 4.1. The quantum mechanical expression, Eq. 4.1 does reduce to the classical case in the low-frequency limit. ${ }^{1}$

\subsection{I-V Curve with Arbitrary Source Admittance}

In a typical experiment, the junction is pumped by a source with a non-zero, usually complex, output admittance. Tucker has extended the theory of photon assisted tunneling to include the case of arbitrary source admittance. Since the input admittance of the junction varies with bias voltage, the pump voltage varies with bias voltage. This causes the pumped $\mathrm{dc} I-\mathrm{V}$ curve to differ from that calculated for the voltage pumped case. With the correct source admittance, we can obtain photon assisted tunneling steps with low or negative dynamic conductance. We will now explain the appearance of this low or negative conductance using the Tucker theory.

As pointed out by Smith et al, the dynamic conductance can be divided into two parts, 55,56

$$
\begin{gathered}
G_{d y n}=\sum_{n=-\infty}^{\infty} J_{n}^{2}(\alpha) \frac{\partial I_{d c}\left(V_{o}+n \hbar \omega / e\right)}{\partial V_{o}}+ \\
\frac{d \alpha}{d V_{0}} \frac{\partial}{\partial \alpha} \sum_{n=-\infty}^{\infty} J_{n}^{2}(\alpha) I_{d c}\left(V_{o}+n \hbar \omega / e\right)
\end{gathered}
$$

The first part is simply the dynamic conductance of the voltage pumped I-V curve. This is almost always positive; it can only be negative near the gap voltage for a junction with a pronounced proximity-effect-induced supergap structure. ${ }^{54}$ The second part is due to the change in pump voltage. The partial derivative with respect to $\alpha$ is always positive below the sum-gap voltage. In order for steps of negative dynamic conductance to occur, the second term must be negative and larger than the first term, i.e. $d \alpha / d V_{0}$ must be large and negative. We will show that this second term is primarily due to the change in the imaginary part of the pump-frequency input admittance of the junction with bias voltage.

In order to facilitate discussion, we use the equivalent circuit shown in Fig. 4.2. The junction is assumed to be driven by a sinusoidal current source $I(t)=I_{\omega} \cos (\omega t)$ with output admittance $Y_{e m b}=G_{e m b}+i B_{e m b}$. The source has an available power $P_{\mathrm{av}}=\mathrm{I}_{\omega}{ }^{2} / 8 \mathrm{G}_{\mathrm{emb}}$. We consider the embedding admittance to be the parallel combination of the admittance due to the local environment of the junction (e.g. the junction mount, any tuning elements present, output admittance of any antenna) and the susceptance due to the geometrical capacitance of the junction. The junction admittance $\mathrm{Y}_{\mathrm{q}}=\mathrm{G}_{\mathrm{q}}+$ 
$\mathrm{iB}_{\mathrm{q}}$ includes only the quanturn admittance, i.e. that due to the quantum mechanical tunneling process. To calculate the pumped I-V curve, we must calculate $V_{\omega}$ at each bias point. Since the quantum admittance of the junction is dependent of the magnitude of the drive voltage, we must selfconsistently solve the equation

$$
\left|I_{\omega}\right|=V_{\omega} Y_{e m b}+V_{\omega} Y_{q}\left(V_{o}, V_{\omega}\right)
$$

with $Y_{q}=G_{q}+i B_{q}$ given by (from Eqs. 3.6 and 3.7)

$$
G_{q}\left(V_{o}, V_{\omega}\right)=\frac{1}{V_{\omega}} \sum_{n=-\infty}^{\infty} J_{n}(\alpha)\left[J_{n+m}(\alpha)+J_{n-m}(\alpha)\right] I_{d c}\left(V_{o}+n \hbar \omega / e\right)
$$

and

$$
B_{q}\left(V_{0}, V_{\omega}\right)=\frac{1}{V_{\omega}} \sum_{n=-\infty}^{\infty} J_{n}(\alpha)\left[J_{n+m}(\alpha)-J_{n-m}(\alpha)\right] I_{k k}\left(V_{0}+n \hbar \omega / e\right)
$$

The quantum admittance used here is the arbitrary signal version of Eqs. 3.8a and $3.8 \mathrm{~b}$. In general, this equation must be solved numerically at each dc bias point.

In order to gain some physical insight into the effects of the quantum admittance on the pumped I-V curve, we first recall the small signal limit discussed in the previous chapter. In this limit, $\mathrm{G}_{\mathrm{q}}$ and $\mathrm{B}_{\mathrm{q}}$ are independent of $\mathrm{V}_{\omega}$. They are plotted as a function of bias voltage in Figs. 3.1c and 3.1d In the region between $\hbar \omega /$ e below the sum-gap voltage and the sum-gap voltage (where the first photon assisted tunneling step will appear in the large signal limit), the quantum conductance is approximately constant. The quantum susceptance, however, decreases rapidly with increasing bias voltage from a relatively small positive (capacitive) value to a relatively large negative (inductive) value. It is easy to see how this change in susceptance is responsible for a variation of pump voltage with dc bias voltage. If $\mathrm{B}_{\mathrm{q}}+\mathrm{B}_{\mathrm{emb}}$ $=0$, no current flows through the susceptive part of the circuit, which leads to a large pump voltage. If $\left|\mathrm{B}_{\mathrm{q}}+\mathrm{B}_{\mathrm{emb}}\right|$ is large, current is shunted through the susceptive part of the circuit, which leads to a small pump voltage. Note that if $B_{e m b}$ is much greater than the variation in $B_{q}$, the change in $B_{q}$ will have little relative effect on $\left|B_{q}+B_{e m b}\right|$, and $V_{\omega}$ will be almost independent of $d c$ bias voltage. This means that a junction with a large geometrical capacitance without any tuning elements will be close to being voltage pumped. For most results in this work, the capacitance is resonated at the pump frequency by an inductive tuning element, so $\left|\mathrm{B}_{\text {emb }}\right|$ less than or on the same order as $\mathrm{B}_{\mathrm{q}}$.

In the large signal limit, the situation become more complicated. The currents at the pump freguency are no longer linearly related to the pump voltage. To calculate the $\mathrm{LO}$ currents, the pump voltage must be calculated numerically at each point, and then substituted into Eqs 3.6 and 3.7 to obtain 
the pump currents. The results of such a calculation are shown in Fig 4.3. Two different values of the embedding admittance are used to illustrate general trends. Notice that the shapes of the junction conductance and susceptance are similar to those calculated in the small signal limit. On any photon assisted tunneling step, the conductance is fairly constant, but changes rapidly between steps. The susceptance, however, changes rapidly on the first sub-gap and the first super-gap steps. Now we will focus our attention on the first sub-gap step, and explain the effects of the embedding admittance on the dynamic conductance of that step.

In the capacitive case $\left(Y_{e m b}=G_{N}+i 2 G_{N}\right.$, where $G_{N}$ is the normal conductance of the junction), $\left|\mathrm{B}_{\mathrm{q}}+\mathrm{B}_{\mathrm{emb}}\right|$ is large at the lower end of the step, and approaches zero at the upper end of the step. This causes the reduced pump voltage to increase across this step. Thus $d \alpha / d V$ is positive, leading to a large dynamic conductance.

In the inductive case, $\left|\mathrm{B}_{\mathrm{q}}+\mathrm{B}_{\mathrm{emb}}\right|$ is finite but small at the lower end of the step, and increases to a large value at the upper end of the step, causing $\alpha$ to decrease across the step. In this case, $\mathrm{d} \alpha / \mathrm{dV}$ is negative and large enough to cause the second term in Eq. 4.2 to dominate the first, and the dynamic conductance on the first step is negative.

Calculations were also carried out using the same dc I-V curve, but ignoring the out-of-phase currents which flow in the junction. Negative dynamic conductance was observed on the calculated pumped dc I-V curves only when the real part of the embedding admittance was unreasonably low ( $<0.001 \Omega^{-1}$ ). The negative conductance only appeared at the extreme low voltage end of the first sub-gap step, in a region actually between the first and secona sub-gap steps. We have never experimentally observed negative conductance in this region.

We therefore conclude that the quantum susceptance in necessary for the production of photon-assisted tunneling steps with low or negative dynamic conductance, at least for the moderate quality junction used in this part of the study. In higher quality junctions with a sharper current rise at the sum-gap voltage, the quantum susceptance is probably even more important because the magnitude of the cusp in the Kramers-Kronig transform of the dc I- $V$ curve would be larger, leading to a larger swing in the quantum susceptance across a step.

\subsection{Fitting of Experimental Pumped I-V Curves}

Since the pumped I-V curve depends on the embedding admittance at the pump frequency, it should be possible to infer the embedding admittance under actual experimental conditions by fitting to measured pumped I-V curves using embedding admittance as a free parameter. There are several methods that can be used. In practice, these methods can only be used when harmonic response is assumed to be shunted (usually by the geometrical capacitance of the junction). All of these methods assume an embedding 
admittance and an available pump power, then calculate the pumped I-V curve based on these parameters, and then compare the shape of the calculated and measured purrped I-V curves. We outline the variations below. These methods are similar to the method of circles developed by Shen ${ }^{57}$ for determining the embedding admittance.

\subsection{1 "Eyeball" Method}

The first method is the so-called "eyeball" technique. In this method, pumped I-V curves are computed for various values of embedding admittance, and after optimizing the available pump power, the shapes of the measured and theoretical are compared by eye. This method is at best tedious and non-quantitative.

\subsubsection{Computerized Current Match}

This method is essentially an automated version of the eyeball technique. Here an admittance is assumed, the available pump power is optimized. Then the sum of the squared differences between the experimental and calculated pumped dc current is calculated for a number of representative bias voltages. The computer can be used to step through a range of admittances to find the best fit. The disadvantage of this method is that a large amount of computer time is required because a Fourier-Bessel series must be inverted for each admittance-bias point.

\subsubsection{Computerized Voltage Match}

The voltage match method ${ }^{58}$ is a more efficient approach. In this method, the experimental pumped IV curve and the measured unpumped IV curve are used to compute the values $V_{k}$ of the pump voltage at several $(\sim 10) \mathrm{dc}$ voltages at which the curves are to be compared. This pump voltage is used to the compute the input admitiance $Y_{k}$ of the junction at the pump frequency at each of the dc voltages. For a given value of the embedding admittance $Y_{e m b}$ we can calculate a new value for each of the pump voltages using the above input admittances,

$$
\mathrm{V}_{\mathrm{k}}^{\prime}=\frac{\mathrm{I}_{\omega}}{\mathrm{Y}_{\mathrm{emb}}+\mathrm{Y}_{\mathrm{k}}}
$$

The optimum values of the embedding admittance and the pump drive current $I_{L O}$ are those that minimize the mean square deviation $\epsilon$ between the $\mathrm{V}_{\mathrm{k}}$ 's and the $\mathrm{V}_{\mathrm{k}}$ ' $\mathrm{s}$,

$$
\epsilon=\sum_{k}\left(V_{k}-\left|V_{k}^{\prime}\right|\right)^{2}=\sum_{k} V_{k}^{2}+\sum_{k}\left|V_{k}^{\prime}\right|^{2}-2 \sum_{k} V_{k}\left|V_{k}^{\prime}\right|=
$$




$$
\sum_{k} V_{k}^{2}+\left|I_{\omega}\right|^{2} \sum_{k} \frac{1}{\left|Y_{e m b}+Y_{k}\right|^{2}}-2\left|I_{\omega}\right| \sum_{k} \frac{V_{k}}{\left|Y_{e m b}+Y_{k}\right|} .
$$

By differentiating this expression with respect to $I_{\omega}$ we obtain the optimum value for $I_{\omega}$, given by

$$
\left|I_{\omega}\right|=\frac{\sum_{k} \frac{V_{k}}{\left|Y_{e m b}+Y_{k}\right|}}{\sum_{k} \frac{1}{\left|Y_{e m b}+Y_{k}\right|^{2}}} .
$$

Substituting this into the expression for $\epsilon$ we obtain

$$
\epsilon=\sum_{k} V_{k}^{2}-\frac{\left(\sum_{k} \frac{V_{k}}{\left|Y_{e m b}+Y_{k}\right|}\right)^{2}}{\sum_{k} \frac{1}{\left|Y_{e m b}+Y_{k}\right|}} .
$$

Here, $\epsilon$ can now be regarded as a parameter quantifying the quality of the fit between the experimental and theoretical pumped I-V curves for a given embedding admittance.

\subsubsection{Automated Fitting}

To illustrate the fitting process, we plot contour maps of the fit quality $\epsilon$ as a function of position on a Smith chart. An example of such a map is shown in Fig. 4.4 In general, when $\epsilon$ is less than $5 \times 10^{-4}(\mathrm{mV})^{2}$, the simulated pumped I-V curves are visuaily indistinguishable from each other and fit the experimental pumped IV curves very well.

\subsubsection{Fit Quality}

An example of a typical fit is shown in Fig. 4.5. To our knowledge, these are the highest quality fits to pumped I-V curves yet obtained. Over the past several years, we have used this procedure to fit hundreds of pumped I$\mathrm{V}$ curves of various SIS junctions. In general we have found that the more closely the dc I-V curve of a given junction resembles that predicted by elastic tunneling theory, the higher the quality the fit obtained. Specifically, 
junctions with substantial leakage currents yield poor fits. Also, junctions whose I-V curves exhibit substantial proximity-effect induced structures immediaiely above the current rise at the sum-gap yield poor quality fits above the sum-gap. Typically, in such junctions, there is a region of negative dynamic conductance, and the measured dc I-V curve does not accurately represent the density of quasiparticle states near the gap because of instabilities in the bias circuit when driving this negative dynamic conductance. We have also fitted pumped I-V curves of series arrays of 5 nominally identical junctions. It has been argued ${ }^{2}$ that such arrays can be treated as a single junction if the measured voltage and current are scaled down by a factor of five. We have found that the fits of pumped I-V curves using such a scaling procedure are of relatively poor quality, even when the junctions in the array have nearly identical critical currents.

\subsubsection{Comparison with Results from a Scaled Model}

To check the validity of the I-V curve fitting technique, we can compare the deduced embedding admittances to those obtained from a scaled model. These measurements were done using the waveguide-based mixer test apparatus to be discussed in chapter 5 . The mixer block has a single adjustable tuning element, and non-contacting backsiorts. We have also constructed an accurate scaled model of this mixer block which allows us to measure the embedding impedance at a scaled frequency range of $6-8 \mathrm{GHz}$. Because of experimental difficulties in our apparatus, it was difficult to relate the position of the backshort in the scaled model to the position of the backshort in the actual experiment. So, instead of comparing the deduced and measured embedding admittances for specific backshort position, we compare the range of embedding admittances accessible by moving the backshort while holding the LO frequency fixed. To do this, we measured a set of I-V curves pumped at $93 \mathrm{GHz}$, each with the backshort position stepped $0.0165 \mathrm{~mm}$ farther away from the junction, starting at approximately $1.15 \mathrm{~mm}$ away from the junction. The range of admittances consistent with each I-V curve was deduced using the voltage match method. For each I-V curve, this range can be represented on the Smith chart as a roughly oval shaped region. These regions, along with the range of admittance measured from the scaled model ${ }^{59}$ at the scaled frequency, are shown in Fig. 4.6.

The deduced admittances are in good qualitative agreement with those measured from the scaled model. The deduced admittances lie on an oval with a larger radius than the circular sange measured from the scaled model. This could be caused by inaccurate scaling, or by differences in the surface impedance between the scaled model and the mixer block at the measured frequencies. It should be noted that $\mathrm{I}-\mathrm{V}$ curves were measured only for a small range of backshort positions. For most positions the admittance lies between region (a) and region ( $k$ ). The pattern is qualitatively repeated with 
period $\sim 1.5 \mathrm{~mm}$, which is approximately $1 / 2$ the guide-wavelength at 93 $\mathrm{GHz}$.

The successful comparison between admittances measured from a scaled model and those deduced from pumped I-V curves gives us confidence to apply the I-V curve method to the modeling (if mixer performance. This will be discussed in chapter 5 .

\subsection{Conclusion}

We have studied the effect of the quantum susceptance in the large signal limit by studying the photon-assisted-tunneling steps with negative conductance. This negative conductance is due to the larger $\mathrm{RF}$ drive voltage caused by the capacitive quantum susceptance at lower bias voltage. The agreement between the I-V curves calculated including the quantum susceptance and the experimental ones is essentially perfect. This result provides an effective way to identify whether the imbedding admittance is inductive or capacitive by observing the slope of the photon-assistedtunneling steps. This method has proved to be very useful in searching the optimum frequencies for SIS mixers coupled to tuning elements. 


\section{Chapter 5 \\ Waveguide Mixer Results Using Tantalum Tunnel Junctions}

\subsection{Introduction}

Heterodyne receivers which use the nonlinear response of SIS tunnel junctions have been shown to provide the lowest noise over a broad range of the millimeter and sub-millimeter electromagnetic spectrum. ${ }^{2,4}$ However, even the best of the receivers have fallen short of the performance which is predicted by the theory of quantum mixing. ${ }^{1,2}$ Because of the lack of detailed comparisons between experimental and theoretical performance, it ras been unclear whether the discrepancy between measured and predicted performance is due to difficulties in coupling the signal to the mixer, or problems with the theory.

Several authors have made quantitative comparisons of SIS mixer performance with theory. Feldman et al. ${ }^{60}$ obtained good agreement with theoretical predictions of mixer gain at $115 \mathrm{GHz}$ using embedding admittances measured from a scaled model. However, they were not able to measure mixer noise accurately enough for a comparison with theory. McGrath et al. ${ }^{61}$ made an extensive comparison between theory and experiment near $36 \mathrm{GHz}$. They concluded that the theory overestimates the gain, and underestimated the noise by a significant amount. They did not measure the embedding admittances involved in the actual experiment, and therefore could only compare experimental performance with that predicted with the embedding admittance optimized for best performance. The ranges of allowable embedding admittance for their work were determined from a scaled model.

In this chapter and more briefly in the letter and paper that preceded it $^{9}, 10$ we carry out a detailed analysis of the performance of high quality, small area (1.0 $\times 1.0$ micron) $\mathrm{Ta} / \mathrm{Ta}_{2} \mathrm{O}_{5} / \mathrm{PbBi}$ tunnel junctions used as quasiparticle mixers near $90 \mathrm{GHz}$. We compare theoretical and experimental pumped I-V curves to deduce accurate embedding admittances under experimental conditions, and use these admittances to predict both mixer noise and mixer gain. These predictions are then compared with experimentally measured values.

This chapter is organized as follows. In sections 5.2, 5.3, and 5.4, we discuss, respectively, the RF, IF and dc measurement apparatus. In section 5.5 we give a description of the tunnel junctions used. In section 5.6 we discuss the measurement scheme. In section 5.7, we show our mixing results, and in 5.8 , we compare these results with the theoretical predictions of the Tucker theory.

\subsection{RF Measurement Apparatus}

A block diagram of the RF apparatus is shown in Fig. 5.1. The local oscillator is a tunable Gunn effect oscillator ${ }^{48}$. The oscillator provided $\sim 20$ 
$\mathrm{mW}$ of $\mathrm{RF}$ power over a tuning range of $78-115 \mathrm{GHz}$. For monitoring purposes, a switch could be used to redirect the RF power to a thermistor based power meter or to a digital frequency counter. The RF power enters the dewar by way of a stainless steel waveguide and is coupled to the mixer block through a 23- $\mathrm{dB}$ crossed-guide coupler. Both the loss in the $80 \mathrm{~cm}$ stainless steel waveguide and the $23-\mathrm{db}$ coupling value of the coupler serve to reduce the amount of room temperature radiation incident on the mixer.

In order to make precise measurements of mixer performance, a calibrated signal must be coupled to the mixer. To do this, we terminate the straight-through arm of the coupler with a specially designed variable temperature RF waveguide load ${ }^{62}$ which provides a calibrated blackbody signal with a spectral density that depends on its temperature. A diagram of this variable temperature load is shown in Fig. 5.2. The load is made from a $250 \mu \mathrm{m}$ thick silicon vane inserted through a narrow slot in the broad wall of silver WR-10 waveguide. The vane was made from a standard 2 inch silicon wafer. The gradual taper of the secion in the waveguide minimized reflection. A nichrome film was evaporated on one face of the vane to provide an absorbing surface. The emissivity was inferred from VSWR measurements to be very close to unity ( 0.999).

The temperature of the load is measured using a germanium resistance thermometer, and the load can be heated using a $500 \Omega$ metal film resistor. The load is thermally isolated from the bath by thin-walled fiberglass tubes. The time constant was set to be $\sim 5$ seconds by choosing the length and diameter of the copper wire thermal link to the helium bath. The internal time constant of the vane is estimated to be less than 1 second at cryogenic temperatures due to the low heat capacity of silicon.

The resistance of the thermometer is read out using a four-wire ac bridge method to avoid errors due to lead resistance and thermal emf's. The thermometer was isolated from external heat sources by using $125 \mu \mathrm{m}$ diameter manganin wires which were well heat sunk at the bath temperature.

The load is connected to the mixer block by the following lossy components: a $76 \mathrm{~mm}$ length of silver waveguide, a $25 \mu \mathrm{m}$ thick mylar window epoxied between two waveguide flanges, a $23 \mathrm{~dB}$ crossed-guide coupler, and a $25 \mathrm{~mm}$ length of silver waveguide. Together these cause an input loss of $0.5 \pm 0.2 \mathrm{db}$ at $1.3 \mathrm{~K}$.

A diagram of the mixer block is shown in Fig. 5.3. The mixer block is made of OFHC copper. Each half of the split block contains one half of a WR10 waveguide channel. A channel-waveguide transformer reduces the waveguide height by a factor of four. A non-contacting $\lambda / 4$ backshort serves as an adjustable tuning element. The SIS tunnel junction, along with an integrated RF band-reject filter pattern is deposited on a $0.9 \mathrm{~mm} \times 12.5 \mathrm{~mm}$ fused quartz substrate which is $0.15 \mathrm{~mm}$ thick. The substrate is placed across the waveguide in a smaller channel forming a suspended stripline circuit 
outside the waveguide. One side of this stripline circuit is connected to the center conductor of an SMA connector, to provide coupling to the IF amplification system.

\subsection{IF Measurement Apparatus}

The IF measurement apparatus must be able to accurately measure the power coupled out of the mixer at the IF frequency, as well as the power reflection coefficient off the mixer at the IF frequency. A diagram of the IF measurement system is show in Fig. 5.4. The IF power from the mixer is coupled to a liquid Helium temperature GaAs high electron mobility transistor (HEMT) amplifier through a quarter-wave microstrip matching transformer, and a cooled L-band isolator. The noise temperature of the HEMT is measured to be $5-6 \mathrm{~K}$ between 1.2 and $1.6 \mathrm{GHz}$. The output of the HEMT amplifier is coupled out of the Dewar using stainless steel coaxial cable and is further amplified by room temperature amplifiers. Also at room temperature is a tunable bandpass filter with a bandwidth of $50 \mathrm{MHz}$ used to define the IF bandwidth and center frequency. The amplified signal can be evaluated using a precision power meter, a spectrum analyser, or a crystal detector. A 20-dB bidirectional coupler is used to inject monochromatic test signals in order to measure the reflection coefficient $|\rho|^{2}$ for the IF power.

By switching a coaxial switch, the input of the IF system can be connected to a variable-temperature coaxial matched load. This load is similar in function to the RF waveguide discussed above, and is used to provide a blackbody signal to calibrate the performance of the IF system.

\section{4 dc Measurement Apparatus}

The dc bias is coupled to the junction through a bias tee incorporated into the IF frequency matching circuit. The bias is provided by a battery powered source with an output impedance (load line) that can be varied from $10 \Omega$ to $10 \mathrm{k} \Omega$. The voltage across the junction and across a monitor resistor in series with the junction (used to measure the bias current) are measured using low-noise instrumentation amplifiers. The bias current can either be held constant, or swept to provide an I-V curve on an oscilloscope or chart recorder. The I-V curve can also be measured using two computer controlled digital voltmeters to provide an accurate digitized I-V curve for theoretical modeling of mixer performance. This dc measurement system was also used for the measurements discussed in chapters 3 and 4 .

\subsection{Tantalum Junctions}

In order to test quantum mixer theory we require the highest quality tunnel junctions possible. To reduce the nois: we require low subgap leakage currents, and in order to see strong quantum effects, we require a sharp current rise at the sum gap voltage. Both of these criteria are met by $\mathrm{Ta} / \mathrm{Ta}_{2} \mathrm{O}_{5} / \mathrm{PbBi}$ junctions fabricated at Yale University. ${ }^{63,64}$ The $\mathrm{Ta}_{2} \mathrm{O}_{5}$ tunnel 
barrier used in these junctions is of extremely high quality because Ta does not form stable suboxides which would degrade barrier quality.

\subsubsection{Fabrication}

The $\mathrm{Ta} / \mathrm{Ta}_{2} \mathrm{O}_{5} / \mathrm{PbBi}$ junctions used for this experiment are small area $\left(1.0 \times 1.0 \mu \mathrm{m}^{2}\right) .64$ A $3000 \AA$ thick $\mathrm{Ge}$ film is thermally evaporated on the 150 $\mu \mathrm{m}$-thick quartz substrate. $100 \AA$ of $\mathrm{Nb}$ and $3000 \AA$ of $\mathrm{Ta}$ are then ion-beam sputtered and patterned by liftoff. The thin $\mathrm{Nb}$ layer nucleates the bcc phase of the Ta base electrode. A chlorobenzene-soak resist process produces a 1 $(\mu \mathrm{m})^{2}$ resist "dot" with an undercut profile necessary for lift-off. $3000 \AA$ of $\mathrm{SiO}$ is then thermally evaporated: lift-off of the resist "dot" defines the junction window in the $\mathrm{SiO}$ film. After patterning the counter-electrode lift-off stencil, the junction is ion-beam cleaned. The exposed $\mathrm{Ta}$ is then oxidized by a dc glow discharge in pure $\mathrm{O}_{2}$ to produce the $\mathrm{Ta}_{2} \mathrm{O}_{5}$ tunnel barrier. Thermal evaporation of $3000 \AA$ of $\mathrm{Pb}_{0.9} \mathrm{Bi}_{0.1}$ and $150 \AA$ of In completes the tunnel junction. The base electrode ion-beam cleaning, oxidation, and thermal evaporation of the counter-electrode are completed in-situ in order to produce a high quality tunnel barrier. The device is completed by lift-off of the counter electrode. All fabrication of tantalum junctions was performed at Yale University by A. H. Worsham.

\subsubsection{I-V curves}

The majority of the experiments reported here were carried out on a single SIS junction. This junction had a normal resistance of $72 \Omega$ at $1.3 \mathrm{~K}$. Both the normal resistance and the shape of the I-V curve remained constant over a period of six months even though for much of that time the junction was stored at room temperature in a desiccator. This durability is in contrast to the behavior of earlier tantalum junctions and is attributed to the $150 \AA$ overlayer of indium deposited on top of the counter-electrode. ${ }^{64}$

The I-V curve of the tantalum junction used in this experiment showed an extremely sharp current rise at the sum-gap voltage as well as extremely low sub-gap leakage current. The voltage width $\Delta \mathrm{V}$ over which the sum-gap current step rises from 0.1 to 0.9 of its full value is less than 0.01 $\mathrm{mV}$. The leakage current at $0.8 \mathrm{~V}_{\text {gap }}$ is less than $0.05 \mathrm{I}_{\mathrm{c}}$. The dc $\mathrm{I}-\mathrm{V}$ curve of the junction is shown in Fig. 5.5.

\subsubsection{Dependence of I-V curves on Magnetic field}

The quasiparticle branches of the dc I-V curves of these junctions depended on applied magnetic field and on the amount of magnetic flux trapped in the junction. When no magnetic field was applied, and for some amount of trapped flux, the current rise at the sum-gap voltage was extremely sharp, and in some cases exhibited negative dynamic conductance. For different amounts of trapped flux, as evidenced by a lower value of the critical current, the sum-gap current rise was not so sharp, and the leakage 
current increased by as much as a factor of two. Two different I-V curves for the same junction at the same temperature are shown in Fig 5.5. Both I-V curves were measured with zero applied field.

For the comparison between measured and calculated mixer performance discussed later in this paper, it was important that the dc I-V curve remain constant throughout the experiment. We found that certain operations (such as switching the electrically controlled coaxial switch) sent transients to the junction which caused the amount of flux trapped in the junction to change. We found that we could regain the original I-V curve by repeatedly switching the coaxial switch, presumably reproducing the original value of the trapped flux..

When an external magnetic field of moderate strength was applied parallel to the plane of the junction, the dc I-V curve changed significantly. $\mathrm{I}-\mathrm{V}$ curves for the same junction at the same temperature but for several different values of the applied magnetic field are shown in Fig. 5.6. Notice that the dynamic conductance of the sum-gap current rise decreased with increasing field, but without a significant rise in leakage current at bias voltages less than $0.8 \mathrm{~V}_{\mathrm{g}}$. This effect is due to a smearing of the density of states as the applied field approaches the critical field of the superconducting films.

\subsection{Measurement Scheme}

Accurate measurements of mixer noise and gain are required in order to evaluate mixer performance. The technique used in this work, which employed variable temperature loads, is described elsewhere. ${ }^{62,65}$ We will summarize the technique here. First we will discuss a simplified version of the measurement scheme we use to measure the mixer performance. Because all quantitative measurements were done using broadband blackbody signals, we use spectral densities (measured in $\mathrm{WHz}^{-1}$ ) to characterize the signal strength. With the local oscillator applied, the spectral density of the IF output signal $\mathrm{S}_{\text {out }}$ is

$$
S_{\text {out }}=G_{r}\left(S_{r}+S_{i n}\right) .
$$

Here $S_{i n}$ is the spectral density of the input signal, $S_{r}$ is the spectral density of the noise added by the receiver (i.e. the total noise added by the mixer and the IF amplification system), and $G_{r}$ is the gain of the receiver. In our case, the input signal incident on the mixer is a single-mode blackbody signal produced by the RF load at temperature $T$ with

$$
S_{\text {in }}=\frac{\hbar \omega}{2} \operatorname{coth}\left(\frac{\hbar \omega}{2 \mathrm{kT}}\right) .
$$

When $\hbar \omega / 2 k T \ll 1$, note that this reduces to $S_{i n}=k T$, as expected in the Rayleigh-Jeans limit for a single-mode source. The measurement scheme is 
as follows. First, we measure $S_{\text {out }}$ as we vary $T$. Then we plot $S_{\text {out }}$ as a function of $S_{i n}$, which we calculate from the measured values of $T$ and Eq. 5.2. The slope of the best linear fit to this plot is the receiver gain $G_{r}$, and the $x-$ intercept gives the negative of the spectral density of the noise added by the receiver. If we switch the coaxial switch so that the IF amplification system is connected to the variable temperature IF load, we can repeat the above procedure to measure the gain $G_{I F}$ and noise spectral density $S_{I F}$ of the IF system. We can rewrite eq. 5.1 in terms of the mixer and IF parameters,

$$
S_{\text {out }}=G_{I F}\left[S_{I F}+G_{m}\left(S_{m}+S_{i n}\right)\right] \text {, }
$$

Where $S_{m}$ is the spectral density of the noise added by the mixer, $G_{m}$ is the gain of the mixer. Its easy to see that we can extract the mixer parameters as

$$
G_{m}=\frac{G_{r}}{G_{I F}} \text {, and } S_{m}=S_{r}-\frac{S_{I F}}{G_{m}} \text {. }
$$

In this simple discussion we have neglected many sources of noise, losses between the RF load and the mixer, and losses due to impedance mismatches in the IF system. A more realistic analysis yields

$S_{\text {out }}=G_{I F}\left\{S_{I F}+\rho_{m}^{2} S_{B}+G_{m}\left(1-\rho_{m}^{2}\right)\left[S_{m}+S_{L O}+\alpha S_{B}+(1-\alpha) S_{i n}\right]\right\}$.

where $G_{m}$ is the gain of the mixer, $S_{m}, S_{L O}$, and $S_{B}$ are the spectral densities of the noise added by the mixer, room temperature noise leaking down the LO waveguide, and the Helium bath respectively. Here $\alpha$ is the loss between the rf load and the mixer, and $\rho_{m}$ is the magnitude of the IF reflection from the mixer due to admittance mismatch. The term $\rho_{m}{ }^{2} S_{B}$ is due to blackbody radiation emitted by the isolator reflecting off the mixer and then coupling to the amplifier.

A mixer test proceeds as follows. First, the receiver performance is measured as described above using the varaible temperature RF load. Then the reflection coefficient from the mixer is measured by injecting coherent signals through the test ports shown in Fig 5.4. When a signal is injected into port one, it is coupled straight out through the isolator and IF amplifier. When a signal is injected into port two, it travels first toward the mixer, where it is reflected, and then couples through the isolator. The power of the two signals is measured using a spectrum analyser, and the ratio between them is the power reflection coefficient of the mixer $\rho_{\mathrm{m}}{ }^{2}$.

We then calculate mixer performance by solving Eq. 5.5 for $G_{m}$ and $S_{m}$,

$$
G_{m}=\frac{G_{r}}{G_{I F}\left(1-\rho_{m}^{2}\right)(1-\alpha)}
$$


$S_{m}=(1-\alpha) S_{r}-S_{L O}-\left[\alpha+\frac{\rho_{m}^{2}}{1-\rho_{m}^{2}}\right] S_{B}-\frac{S_{I F}}{G_{m}\left(1-\rho_{m}^{2}\right)}$

In a typical experiment, four or five values of the rf load temperature were chosen in the range from $1.3 \mathrm{~K}$ to $20 \mathrm{~K}$ and the output power measured for each load temperature. A least squares linear fit to these points was used to compute the mixer performance.

\subsection{Mixer Optimization}

Mixer performance is dependent on a large number of experimental parameters. These include, but are not limited to, dc bias point, applied local oscillator power, local oscillator frequency, IF frequency, backshort position, temperature, and applied magnetic field. Before the mixer performance is measured using the above procedure the appropriate parameters were optimized to maximize the coupled gain $G_{c}=\left(1-\rho_{m}{ }^{2}\right) G_{m}$. This was typically done by injecting a monochromatic signal through the LO waveguide at either the upper or lower sideband and maximizing the output power $P_{I F}$ of the IF system, which was monitored with a direct detector.

\subsection{Mixer Performance}

The coupled gain and mixer noise for our mixer are plotted as a function of local oscillator frequency in Fig. 5.7. The backshort position and available local oscillator power were optimized for each frequency. The minimum mixer noise was found to be $0.61+/-0.36$ quanta at $93.0 \mathrm{GHz}$. The sideband ratio for this operating point was $9.8 \mathrm{~dB}$, essentially making this a single sideband (SSB) mixer. The mixer noise is within 25 percent of the SSB quantum limit of 0.5 quanta. This is, to our knowledge, the closest approach to the quantum limit ever demonstrated.

The coupled (transducer) gain was always measured to be less than unity. Simulation has shown that DSB mixers with such sharp I-V curves can give values of coupled gain much greater than unity. We attribute our low gain to the small instantaneous bandwidth of our mixer mount at the RF frequency. This matter will be discussed in more detail later in the thesis.

\subsection{Comparison with Theory}

In order to compare our experimental results with the Tucker theory, we have carried out computer simulations of mixer performance. All calculations were done using the three-port model that is, with currents generated at the first and higher harmonics assumed to be short-circuited. This is a reasonable assumption in our case because the relatively large geometrical capacitance of the junction $\left(\mathrm{C} \sim 160 \mathrm{fF}, \omega \mathrm{R}_{\mathrm{N}} \mathrm{C}=14\right.$ at $190 \mathrm{GHz}$ ) of the junction shunts currents at harmonic frequencies. 
The Tucker theory provides a method for predicting the highfrequency properties of a quasiparticle mixer from the dc I-V curve, provided that both the dc I-V curve and the rf performance are determined only by elastic tunneling processes. Because the I-V curves of our junctions closely resemble the $\mathrm{I}-\mathrm{V}$ curves calculated from the $\mathrm{BCS}$ density of states and elastic tunneling theory, we expect that the dc I-V curve is largely determined by elastic tunneling events. Many other junctions exhibit sub-gap currents substantially in excess of those predicted by the BCS/elastic tunneling theory. These currents may not arise from elastic tunneling, and hence would not be correctly modeled by the Tucker theory. If this is the case, our junctions should be a favorable case for a quantitative test of the Tucker theory.

\subsubsection{Determination of Embedding Admittances}

To calculate mixer performance from the Tucker theory, we must know the embedding admittance both at the upper sideband frequency, and at the lower sideband frequency. These admittances can be determined in several ways. First, numerical modeling of the embedding structures could be carried out. While this may be possible in simpler situations, the complexity of our mixer block would make this process tedious and unreliable. We have instead used two other approaches. The first is to measure the admittance of a large scaled model of our mixer block at lower frequencies $(3-10 \mathrm{GHz})$ where accurate network analyzers are available. We have also determined the embedding admittance by studying the shape of the pumped I-V curve at various frequencies and backshort positions. Comparisons between these two methods show good agreement. Because of the large amount of backlash present in the backshort drive, it was impossible to determine the exact admittance present under specific experimental conditions. We therefore deduced the embedding admittance by studying the shapes of the pumped I$\mathrm{V}$ curves measured under experimental conditions.

It has long been known that the embedding admittance at the pump frequency influences the shape of the pumped I-V curve. The nature of this dependence was discussed in the chapter 4 . Other workers have used the shape of the pumped I-V curve to determine in general the range of embedding admittance provided by their mixer mounts. ${ }^{58,66,67}$ This work utilizes the shape of the pumped I-V curve to deduce the embedding admittances present under actual experimental conditions which are needed to accurately model mixer performance.

\subsubsection{Simulation of Mixer Performance}

We have calculated mixer performance using the Tucker theory in the 3-port approximation. We have not made the low IF approximation used by some other authors. The input data are the dc I-V curve, the bias voltage, the LO voltage amplitude $V_{L O}$, the RF embedding admittances $Y_{\text {usb }}$ and $Y_{1 s b}$ at the upper and lower sideband frequencies respectively, and the IF load 
admittance $\mathrm{Y}_{\mathrm{IF}}$. We consider the susceptance due to the geometrical junction capacitance to be part of the embedding admittance.

To predict mixer performance we must measure or deduce the above input data under experimental conditions. The $d=I-V$ curve and the bias voltage are straightforward to measure. The LO voltage amplitude was determined from the pumped dc current at the bias point using the Tucker theory. The IF load admittance was assumed to be matched to the mixer IF output admittance, yielding the available gain.

The RF embedding admittances were determined by pumping the mixer first at the upper sideband frequency, and then at the lower sideband frequency, and measuring a pumped I-V curve for each case. Frcm these I-V curves, we deduced a range of embedding admittances consistent with each of these I-V curves. Ranges deduced using different available pump power were consistent. In general, the best defined ranges were obtained wher: the available pump power was such that the pumped dc current on the first photon-assisted-tunneling step below the sum-gap was $1 / 4$ to $1 / 3$ of the unpumped current immediately above the sum-gap. The admittances used in this work were deduced from I-V curves measured under these conditions.

The ranges of admittances deduced were used in the Tucker theory to predict a range of mixer performance. This was done by exhaustively sampling on a grid of admittance pairs consistent $\left(\epsilon \leq 5.0 \times 10^{-4}(\mathrm{mV})^{2}, \epsilon\right.$ defined as in Eq. 4.8 ) with the shape of the pumped I-V curves. The range of performance we quote was obtained by plotting a histogram of calculated available gain and mixer noise value, and noting the range into which 90 percent of the predicted values fell.

\subsubsection{Results vs. pump power}

As a first test of this procedure, we analyzed the results of a relatively simple experiment. We measured mixer noise and available gain as a function of LO power with all other parameters (i.e. LO frequency, backshort position, dc bias point, IF frequency, magnetic field, and temperature) held constant. This implies that the embedding admittances at the upper sideband and at the lower sideband were constant during the experiment, and that changes in mixer performance were due only to changes in the amplitude of the LO voltage.

In Fig. 5.8, we plot the experimentally measured mixer noise and available gain as a function of available pump power. The range of predicted performance consistent with the I-V curve is indicated by the dashed lines in Fig. 5.8. The experimental values are consistent with the predicted range of performance, but are at the poor performance end of the range. This is consistent with the conclusions reached by McGrath et al. ${ }^{61}$ For one specific set of embedding admittances within the allowable range, we are able to predict performance that is in nearly perfect agreement with the 
experimentally measured values. The comparison is represented by the solid lines in Fig 5.8. These values of the embedding admittance are identical to those used to produce the fits to the pumped I-V curves shown in Fig. 4.5.

\subsubsection{Results vs. Backshort position}

We have also modeled mixer performance as function of backshort position. We measured mixer gain and noise for 13 different backshort position with LO frequency, IF frequency, and applied magnetic field held constant. The chosen positions were on either side of the optimum backshort position. At each backshort position, the LO power and dc bias point were optimized for maximum coupled gain.

The analysis of this experiment is slightly more complicated because the embedding admittance changes with backshort position. For each backshort position, we measured I-V curves pumped at the upper and lower sideband frequencies and used these to deduce ranges of allowable embedding admittances, which were used to model mixer performance. In Fig. 5.9 we plot the experimentally measured mixer noise and available gain as a function of backshort position. The range of predicted performance is indicated by the dashed lines in Fig. 5.9. The experimental values are in good agreement with the predicted range of performance for backshort positions closer to the junction than $0.59 \mathrm{~mm}$. When the backshort is farther away, the agreement is not as good.

The admittances deduced when the backshort is farther than $0.59 \mathrm{~mm}$ from the junction are on the extreme outer edge of the Smith chart, where either or both the real or imaginary parts of the embedding admittance is much larger than the input admittance of the junction. Under these conditions, the change of the input admittance of the junction with bias voltage does not have a large effect on the LO voltage, and hence I-V curve with different embedding admittances are quite similar. However, admittances that yield almost identical I-V curves have different values of $\mathrm{RF}$ reflection coefficient, and hence different mixer performance.

As the embedding admittance moves radially outward near the edge of the Smith chart, the power needed to ubtain a specific pumped dc current increases rapidly. It is possible to eliminate some of the embedding admittances that are consistent with I- $\mathrm{V}$ curve shape, but inconsistent with the measured value of the LO power. Since the LO power is measured outside the dewar, we must know the loss between the power meter and the junction at the pump frequency. We were able to estimate this loss by using the calculated pump power for the I-V curves measured when the backshort is closer than $0.59 \mathrm{~mm}$ to the junction. For these backshort positions, the embedding admittance is nearer the center of the Smith chart and the pump power varies only by a factor of two over the range of admittance consistent with I-V curve shape.

We used this procedure to restrict the range of admittances when the backshort is more than $0.59 \mathrm{~mm}$ from the junction. In Fig. 5.10 , we replot the 
experimentaily measured mixer noise and available gain. The range of predicted performance calculated using the restricted range of admittances is indicated by the dashed lines. The agreement between experiment and theory is substantially improved over the range in which the corrected admittances were used. It should be noted that the performance calculated using the restricted range of admittance falls outside the range calculated using the unrestricted admittances. This is because the values calculated using the restricted admittances fell outside the 90 percent range used in Fig. 5.9.

\subsubsection{Discussion}

It is useful to consider effects that could cause discrepancies between calculated and experimental mixer performance. It is possible that the Tucker theory overestimates the performance when the dc I-V curve is used to predict high-frequency behavior. This could occur if the dc I-V does not accurately represent the density of states. A very small negative dynamic resistance observed on the sum-gap current rise indicates that the high current density of our junctions heats the quasiparticles and sharpens the current rise at the sum-gap voltage. The time scale of this effect is much longer than one cycle of the local oscillator, so that the high-frequency response is not exactly determined by the dc I-V curve. A second possibility is that the leakage current below the sum-gap does not arise from tunneling, and so is not correctly modeled by the Tucker theory. If this effect were important it could explain our relative success because the effect would be minimized in low-leakage junctions. It is possible that the determination of embedding admittance using pumped I-V curves gives incorrect results, either due to non-equilibrium phenomena, leakage currents, or other effects. We consider this unlikely because of the good agreement between the admittances deduced by the fitting procedure and those measured using a scaled model, ${ }^{59}$ or theoretical expectations. ${ }^{8}$

It is also possibie that harmonic effects are important for some backshort positions. This is unlikely to be the case for the first experiment where performance was measured as a function of pump power. In this experiment, the junction capacitance was nearly resonated at the LO frequency. Under these conditions, the amplitude of the RF voltage at the first harmonic of the pump frequency is $\sim 100$ times smaller than that at the pump frequency for typical pump power, and the assumptions of the threeport model should be very well justified. For the second experiment, where the backshort position is varied, the embedding admittance was highly capacitive at the pump frequency when the backshort was farther than $0.59 \mathrm{~mm}$ from the junction. Under these conditions, voltages at the first harmonic of the pump frequency could arise either from harmonic conversion in the $\mathrm{m}$ cer itself, or from harmonic content in the waveform of the local oscillator. The pump power needed at these backshort positions is up to 100 times larger than when the mixer is optimized, thus there is up to 
100 times more power at the first harmonic frequency. It is possible that the 3-port model is no longer valid under these conditions.

We now turn the discussion to the relatively low values (less than unity) of coupled gain that we measured. Simulations have shown that junctions similar to ours can give large values of coupled gain if provided with the correct embedding admittances. The small instantaneous bandwidth of our mixer block limits our ability to simultaneously provide favorable embedding admittances at both the upper and lower side band. Simulation has shown that as the difference between the imaginary parts of the upper and lower side band embedding admittance increases, the coupled gain decreases for typical mixer parameters. Coupled gain much greater than unity was measured in a full-height version of the mixer block used in this work. ${ }^{68}$ The junction used in that experiment used an integrated tuning element to resonate the geometrical capacitance of the junction which greatly increased the instantantous bandwidth, making the mixer almost double-sideband.

We have accurately measured the performance of an SIS mixer operating in the quantum limit where the noise is limited by the uncertainty principle. Our minimum mixer noise is a maximum of 0.42 quanta above the quantum limit for a phase-preserving linear amplifier. This is, to our knowledge, the closest approach to the quantum limit measured in any mixer. We have calculated pumped I-V curves in nearly perfect agreement with those measured in the experiment for a broad range of experimental parameters. Using admittances deduced from the fitting parameters and the Tucker theory of quantum mixing, we have predicted mixer performance in good agreement with that measured experimentally. 


\section{Chapter 6 \\ Measurements of Quasioptically Coupled SIS Mixers}

\subsection{Introduction}

One of the major goals in the development of SIS mixers is to increase the frequency at which they can be operated with useful sensitivity. Tucker's theory predicts sensitive operation up to frequencies near the sum-gap frequency $(\omega \sim 2 \Delta / \hbar)^{69,70}$, about $600 \mathrm{GHz}$ for mixers using all $\mathrm{Nb}$ junctions. Above $\sim 300 \mathrm{GHz}$, the sensitivity of existing receivers falls well short of that predicted by theory. At least part of this low sensitivity is due the difficulty of coupling the signal to the mixer at submillimeter frequencies.

SIS mixers operated at millimeter-wave frequencies can be coupled to the incoming radiation using waveguide-based coupling schemes. Such a scheme was used successfully in the work discussed in the previous chapter. As the operating frequency is increased into the submillimeter band, in becomes increasingly difficult to fabricate waveguide structures with sufficient accuracy using conventional techniques. The smaller waveguide dimensions also makes it necessary to fabricate the mixer junctions on thinner substrates to prevent excessive dielectric loading of the waveguide. These thin substrates $(\sim 20 \mu \mathrm{m}$ at $500 \mathrm{GHz})$ are difficult to handle and mount with sufficient accuracy. Although waveguide-based mixers have been successfully operated up to $345 \mathrm{GHz}^{71}$, this is probably near the limit of conventionally-fabricated waveguide mixers. There are two possible solutions to this problem. One is to fabricate waveguide structures using micromachining techniques with integrated mixer junctions. ${ }^{72}$ The other is to couple the radiation to the mixer using a quasioptical coupling scheme.

In a quasioptical scheme, optical components such as lenses and mirrors are used to focus the radiation onto an antenna which then couples the radiation to the mixer. A planar antenna can easily be fabricated using microlithography techniques to the accuracy required for sub-millimeter operation. SIS receivers using planar lithographed antennas and quasioptical coupling schemes have shown good performance at millimeter and submillimeter wavelengths. ${ }^{73-75}$

Another of the major problems encountered at near-millimeter and sub-millimeter wavelengths is capacitive roll-off, which can significantly degrade the coupling of the RF signal to the mixer. It may be possible to use high current density $\left(j_{c} \geq 10 \mathrm{kA} / \mathrm{cm}^{2}\right)$ small area $\left(\leq 1 \mu \mathrm{m}^{2}\right)$ junctions so that the capacitance does not play a dominant role in the RF coupling. Such junctions are extremely difficult to fabricate. Also, mixers with extremely small capacitance are prone to complication by harmonic mixing, since the higher harmonics are not shunted by the junction capacitance. These harmonic effects are thought to degrade mixer performance ${ }^{69}$. The highest performance millimeter wave mixers built to date have capacitances such 
that $\omega R_{N} C \sim 4-8$, and use tuning elements to resonate the capacitance at the operating frequency. In a waveguide-mounted mixer, the capacitance can be resonated at the signal frequency using adjustable tuning elements such as backshorts or screw tuners. For quasioptical receivers such adjustable tuning elements are not available or poorly understood. However, it is possible to fabricate lithographed inductive tuning elements which resonate the capacitance at the signal frequency.

In this chapter we discuss development we have done on quasioptical coupling to submillimeter mixers. This chapter is organized as follows: First we discuss the mixer test apparatus we used, including the quasioptical elements and planar antennas. Then we motivate the use of tuning elements to resonate the junction capacitance. We then discuss the use of a Fourier transform spectrometer to rapidly evalute the performance of tuning elements. We then discuss the design and measured performance of each type of tuning structure sequentially. Since the performance of the mixers discussed in this chapter is so far from the quantum limit, we report the noise added by the mixer by citing the noise temperature of the mixer.

\subsection{Mixer Test Apparatus}

The mixer test apparatus shown in Fig. 6.1, allows us to measure the coupled gain and added noise of the mixer under test. The measurement scheme is similar to that discussed in sections 5.2-5.4. The main difference is that the RF black-body source is outside the cryostat. The RF black-body is either a piece of millimeter-wave absorbing foam ${ }^{76}$ immersed in liquid nitrogen $(77 \mathrm{~K})$ or an identical piece of foam at room temperature $(\sim 300 \mathrm{~K})$. We note the output of the IF amplifier when each of these loads is placed at the input of the mixer test system, and from this deduce the gain and added noise of the test system as a whole. As in chapter 5, we must account for the contribution to these parameters from the IF amplification system in order to determine the performance of the mixer.

Returning our attention to Fig. 6.1, the local oscillator is either a Gunn effect oscillator (near $90 \mathrm{GHz}$ ), or a Gunn oscillator pumped reverse-biasedSchottky varactor multiplier (at 180, 270 and $360 \mathrm{GHz}$ ). The local oscillator is coupled to free space using a horn antenna and lens combination which provides a parallel beam. The local oscillator and the signal are combined using a $0.075 \mathrm{~mm}$-thick mylar beamsplitter, which has a transmittance of 0.97 at $90 \mathrm{GHz}$ for the polarization of interest. The combined LO and signal are focused by a $10 \mathrm{~cm}$-diameter, $\mathrm{f} / 1$ off axis paraboloid onto the window of the cryostat. The vacuum window of the cryostat is a $16 \mathrm{~mm}$-diameter $0.02 \mathrm{~mm}$ thick polypropylene film. The window is curved by the external air pressure which reduces Fabry-Perot resonances. A $12.7 \mathrm{~mm}$-diameter $0.83 \mathrm{~mm}$-thick fused quartz window with a $100 \mu \mathrm{m}$-thick black-poly film is attached to the $77 \mathrm{~K}$ heat shield. This window absorbs most of the radiation above the midinfrared range (to reduce radiation loading of the LHe temperature cold plate 
of the cryostat) yet has almost $100 \%$ transmission at small multiples of 90 GHz. Inside the cryostat the LO and signal are further focused by an on-axis ellipsoidal mirror onto a hemispherical quartz lens. The quartz lens provides good thermal contact between the LHe temperature cold plate and the junction. It also provides a dielectric half-space through which the antenna couples most efficiently to the input radiation. The planar antenna and mixer junction are located on the flat side of the hemispherical lens.

The on axis configuration of the ellipsoidal mirror suffers from center obscuration by the mixer mount, so we later changed to a simpler configuration using a hyperhemispherical quartz lens followed by a second TPX lens which couples efficiently to a parallel beam at the dewar window. To accommodate this beam, the dewar window was enlarged to $31.5 \mathrm{~mm}$ and the off axis paraboloid was eliminated from the system.

We have used several planar antennas in our work. We first used a $90^{\circ}$ bowtie antenna. The antenna pattern of this antenna suffers from large sidelobes at $60^{\circ}$ from normal and thus would not couple well to the nearly gaussian beam emitted by a millimeter-wave radio telescope. We have also used the circular-tooth log-periodic antenna shown in Fig. 6.2a. Both the bow angle and the tooth angle are $45^{\circ}$, and ratio of the linear dimension of the adjacent teeth is 2 . The choice of angle and ratio is a compromise between beam pattern and cross polarization. This antenna is designed to be operated between $20 \mathrm{GHz}$ and $1 \mathrm{THz}$. The beam pattern of this antenna is nearly gaussian. One disadvantage of this antenna is that the polarization coupled to the antenna is frequency dependent. We have also used a two-arm logspiral antenna, shown in Fig 6.2b. The antenna pattern of this antenna is nearly gaussian and frequency independent. The radiation coupled to a logspiral antenna is circularly polarized.

All of the antennas used have a self-complementary structure, i.e. the pattern of the metallized part of the substrate is the same as the pattern of the unmetalized part. It has been shown that antennas with this property have a frequency-indepedent admittance which depends on the dielectric constant of the dielectric half space on which it is mounted. ${ }^{77}$ For quartz substrates with $\epsilon_{\mathrm{r}}=3.83$, the antenna admittance is $0.083 \Omega^{-1}$. Because the antenna admittance does not depend of frequency, the task of designing tuning elements is much simplified.

Care has been taken to ensure that the junction is well heat sunk to the cold plate through the quartz hemisphere and its OFHC copper support. A thin layer of vacuum grease was placed between the junction substrate and the lens to increase the thermal contact. We measured the temperature at the center of the antenna using a germanium resistance thermometer. For a $1.8 \mathrm{~K}$ bath temperature, it is $2.6 \mathrm{~K}$ with the HEMT amplifier off and $2.8 \mathrm{~K}$ with the HEMT amplifier on. 
The IF system is identical to that discussed in section 5.3. The overall noise temperature of the IF system, including contributions due to the isolator, is typically $6-7 \mathrm{~K}$.

\subsection{Integrated Tuning Elements}

Before we discuss specific tuning schemes, it is useful to introduce a simplified equivalent circuit and the $R F$ coupling coefficient $C_{R F}$. The equivalent circuit we use is shown in Fig. 6.3. The RF input is represented by an $R F$ current source in parailel with the admittance of the antenna. The junction capacitance, the inductance of the junction leads, and the admittance of any tuning element are represented by $C, L_{L}$, and $X$. The non-linear quasiparticle tunneling is approximated by the $R F$ conductance $G_{R F}$. In reality, this conductance is a complicated function of dc bias voltage and pump power. Under typical operating conditions (on the first photon step), it is approximated by

$$
\mathrm{G}_{\mathrm{RF}}=0.7 \times \mathrm{G}_{\mathrm{N}}(\hbar \omega / \mathrm{eV}) \text {, }
$$

where $G_{N}$ is the normal resistance of the junction, and $V_{g}$ is the sum-gap voltage. Note that we have ignored any effect of the quantum susceptance. The quantum susceptance only shifts the resonant frequency by at most $20 \%$, as seen in chapter 3 .

In general, the conversion gain is a very complicated function of imbedding admittance, dc bias point, and pump power. To a rough approximation, the performance of the mixer is good (high gain, low noise) when the RF coupling coefficient $C_{R F}$ is high, where

$$
C_{R F}=1-\left|\frac{Y_{A}-Y_{j}^{*}}{Y_{A}+Y_{j}}\right|^{2} \text {. }
$$

Here, $Y_{A}$ is the admittance of the antenna, and $Y_{j}$ is the admittance of the right side of Fig. 6.3. $C_{R F}$ is the ratio of the power available at the ariennaterminals to the power absorbed by the quasiparticle conductance $G_{R F}$. (Note that susceptive elements which appear in parallel can be considered to be part of the antenna $\left(Y_{A}\right)$ or part of the junction $\left(Y_{j}\right)$ without changing $C_{R F}$, as we expect physically. This is due to the complex conjugation in the numerator of equation 6.3. The quantity that is mod-squared is not the voltage reflection coefficient.)

All of the tuning element discussed below make use of microfabricated superconducting microstrip transmission lines ${ }^{78,79}$. These transmission lines have typical linewidths of $2-10 \mu \mathrm{m}$ and inter-conductor spacings of 0.5 $\mu \mathrm{m}$. Millimeter-wave transmission lines of this size made from normal metal would be both lossy, due to the surface resistance of the copper, and highly dispersive, due to either the normal or anomalous skin effect, depending on the temperature of operation. Such properties limit their usefulness in the high-Q resonant circuits needed to resonate the junction 
capacitance. Superconductive transmission lines are expected to be nearly lossless and dispersion free for millimeter-wave frequencies and thus should be ideal for the fabrication of integrated tuning elements. Superconducting transmission lines can become lossy, however, if the dielectric separating the two superconductors is lossy, or if the superconducting films themselves are of low quality and have excessive high frequency surface resistance.

The junctions used for the majority of the work reported here are $1.7 \times$ $1.7 \mu \mathrm{m} \mathrm{Nb} / \mathrm{NbO}_{\mathrm{x}} / \mathrm{Pb}-\mathrm{In}-\mathrm{Au}$ junctions. The capacitance of these junctions is estimated to be $160 \mathrm{fF}$. The normal resistance is typically $50-200 \Omega$. Without tuning elements, the $R F$ coupling coefficient $\left(C_{R F}\right)$ is very small, about 0.08 at $90 \mathrm{GHz}$, and correspondingly worse at higher frequencies. Tuning elements are required for acceptable mixer performance. The normal resistance of these junctions is such that good coupling can be achieved if the junction capacitance can be resonated at the frequency of operation.

\subsection{Tests of Tuning Elements Using a Fourier Tranform Spectrometer}

One of the main problems encountered when designing mixers with integrated tuning elements is that the parameters of the junction (such as the normal resistance and capacitance) and of the microstrip transmission lines (such as phase velocity) used are difficult to control precisely. Consequently it is important to be able to measure the frequency response of the mixer structures easily so we can reject those with undesirable frequency characteristics. This often requires measurements over a broader range than are convenient with millimeter and submillimeter wave oscillators. In this section we describe a new method of rapidly measuring the frequency response of quasioptical mixer structures over a frequency range from below $50 \mathrm{GHz}$ to over $500 \mathrm{GHz}$. In this method we couple the output of a Fourier transform spectrometer to the antenna and use the SIS junction as a direct detector (or videodetector) of the coupled power. This is the same technique we used to measure the quantum susceptance in Chapter 3.

The Fourier spectrometer takes the place of the sweep oscillator conventionally used for frequency response tests at lower frequencies. This direct spectroscopic technique has several advantages over the previously used technique of the resonance induced Josephson steps (or Fiske modes) ${ }^{74,79,80}$. The Fiske modes are well defined only for narrow RF bandwidths and are hard to interpret to find bandwidth information. With this new method, we can measure both the resonant frequency and the bandwidth of the resonance.

The spectrum measured by the spectrometer is the product of the frequency-dependent responsivity $S(\omega)$ of the junction operated as a videodetector, the $R F$ coupling coefficient $C_{R F}$, and the spectrum of the radiation source of the spectrometer, which is modified by the frequencydependent beamsplitter efficiency $\eta(\omega)$. Since we are measuring $C_{R F}$, we need 
to understand each of the other terms in order to account for their influence on the measured spectrum.

\subsubsection{The SIS Direct Detector}

The current responsivity of a direct detector is defined as the induced change in DC current per unit RF signal power absorbed in the detecting element. For an SIS junction it is given $b^{2}$

$$
S\left(\omega, V_{d c}\right)=\frac{e}{\hbar \omega}\left[\frac{I_{d d}\left(V_{o}+\frac{\hbar \omega}{e}\right)-2 I_{d d}\left(V_{o}\right)+I_{d d}\left(V_{o}-\frac{\hbar \omega}{e}\right)}{I_{d d}\left(V_{o}+\frac{\hbar \omega}{e}\right)-I_{d d}\left(V_{0}-\frac{\hbar \omega}{e}\right)}\right]
$$

For a typical SIS junction with a moderately rounded I-V curve, such as the one shown in Fig $6.4 \mathrm{a}, \mathrm{S}\left(\omega, \mathrm{V}_{\mathrm{dc}}\right)$ peaks at about $\hbar \omega / \mathrm{e}$ below the sum-gap voltage. The position of this peak shifts away from the gap voltage as the frequency is increased. If we fix the bias voltage at a fixed voltage $V_{0}$, then $S$ will be a function of frequency. In Fig. 6.4b, we plot curves of $S$ as a funtion of frequency at several different bias voltages $V_{0}$ computed using equation 6.10 from the $\mathrm{I}-\mathrm{V}$ curve shown in Fig 6.4a. We can see that the current responsivity reaches its maximum at $\omega_{0}=e\left(V_{g}-V_{0}\right) \hbar$, and then falls as $\omega^{-1}$ at high frequency. Above $\omega_{0}$ the responsivity is a relatively smooth function of frequency.

\subsubsection{Source Spectrum}

The output of the Fourier transform spectrometer is black-body radiation from a $\mathrm{Hg}$-arc lamp. Only a single mode of this radiation is coupled to the antenna so that, in the Rayleigh-Jeans limit, the spectrum is flat. This flat spectrum is modified by the efficiency of the $250 \mu \mathrm{m}$ mylar beamsplitter. The beamsplitter efficiency is a smooth function of frequency as shown in Fig 6.4d.

The dc current produced by the $\mathrm{RF}$ radiation is a product of the responsivity $S\left(\omega, V_{0}\right)$, the RF coupling coefficient $C_{R F}$, and the efficiency of the beamsplitter. Since $S$ is a function of dc bias voltage $V_{0}$, we expect the relative heights of resonances at different frequencies to vary as it is changed. For the narrow fractional bandwidths that we typically measure, however, the frequency dependence of $C_{R F}$ dominates that of $S$ so the resonant frequencies and bandwidths can be easily obtained.

For the experiments discussed in this section, we used a far-infrared Fourier Michelson interferometer operated in the step-and-integrate mode. Since the response speed of the SIS junciton is high, a rapid-scan Fourier spectrometer could also be used. The high sensitivity of SIS direct detectors permits data to be obtained at much lower frequencies than are usually obtained using Fourier spectroscopy. 
Coupling from the spectrometer was accomplished by placing the $11 \mathrm{~mm}$ diameter lightpipe, which provides the output of the spectrometer, against the window of the cryostat. The beam diameter of the output beam from the receiver is estimated to be $\sim 12 \mathrm{~mm}$ at this point, so the light pipe beam is efficiently coupled to the receiver.

The junction voltage at the $17.7 \mathrm{~Hz}$ chopping frequency of the spectrometer is lock-in detected and digitized for Fourier transformation. Although the output impedance of the detector was typically $1 \mathrm{k} \Omega$, so it was not well matched to our FET preamplifier, the detected signal was typically $10^{3}$ times the broadband noise in a $1 \mathrm{~Hz}$ bandwidth, so that adequate signalto-noise ratio could be obtained with integration times of 1-3 s. Performance could be somewhat improved by using an impedance matching transformer.

\subsection{Design and Measurement of Tuning Elements}

In this section we discuss the design and measurement of each type of tuning element we have designed. The first three types are inductive tuning elements placed in parallel with the mixer junction. The junction is located at the terminals of the planar antenna. The last type of tuning schemes use microstrip impedance transformer. In this case, the junction is located some fraction of a wavelength away from the antenna terminals on one of the halves of the antenna.

\subsubsection{3i/8 Stub (Open Ended Stub)}

The first type of tuning element used is an open-ended superconducting microstrip stub, as shown in Fig. 6.5. This stub is fabricated in parallel with the SIS junction. The admittance of an open ended stub constructed from lossless transmission line is given by

$$
Y=i Y_{0} \tan (\beta \ell),
$$

where $\beta$ is $\omega / v, \ell$ is the length of the stub, $Y_{0}$ is the characteristic admittance of the microstrip, $\omega$ is the frequency of the radiation, and $v$ is the phase velocity of signals on the stub at frequency $\omega$. For $n \lambda / 2+\lambda / 4<\ell<n \lambda / 2+$ $\lambda / 2$, where $\lambda$ is the wavelength of the radiation in the microstrip, and $n$ is an integer, this admittance is inductive, and can thus be used to resonate the geometrical capacitance of the junction. The series inductance due to the junction leads is so small that it can be neglected for both the open-ended and shorted-end (section 6.5.2) stubs. The resonance condition is given by

$$
\omega_{0} C+Y_{0} \tan \left(\omega_{0} l / v\right)=0
$$

where $C$ is the capacitance of the junction, and $\omega_{0}$ is the resonant frequency. Note that there are an infinite number of resonances, though each successive 
resonance has a smaller bandwidth. The dimensions of the stub are chosen to satisfy $\omega_{0} C / Y_{0}=1$, which maximizes the bandwidth of the first resonance.

We fabricated mixer junctions with tuning circuits having resonant frequencies of $90,180,270$, and $360 \mathrm{GHz}$. Those are the frequencies at which local oscillators were readily available. First we discuss the results at $90 \mathrm{GHz}$, and then move to higher frequency. The microstrip stubs at all frequencies are constructed from a $\mathrm{Pb}-\mathrm{In}-\mathrm{Au}$ film separated from a $\mathrm{Nb}$ groundplane by $300 \mathrm{~nm}$ of thermally evaporated $\mathrm{SiO}^{79}$.

At $90 \mathrm{GHz}$, we use a $0.38 \mathrm{~mm}$-long stub with a characteristic admittance of $0.1 \Omega^{-1}$. $v_{\text {ph }}$ was estimated to be $0.30 c^{78}$ and the junction capacitance $C$ was estimated to be $0.160 \mathrm{pF}$. In Fig. $6.6 \mathrm{~b}$, we plot the expected $C_{R F}$ calculated assuming a lossless, dispersion free transmission line, and $G_{R F}=Y_{A}=0.083$ $\Omega^{-1}$. The length and characteristic admittance of the line are chosen so that the first resonance occurs at $110 \mathrm{GHz}$. The next two resonances occur at 244 and $387 \mathrm{GHz}$.

In Fig 6.6a, we plot the response of the junction and tuning circuit measured using the Fourier transform spectrometer. The junction was biased at $2.0 \mathrm{mV}$, which is $\sim 0.9 \mathrm{mV}$ below the sum-gap voltage. The spectrum was measured with a resolution of $3 \mathrm{GHz}$. There are 3 distinctive peaks in the spectrum at 110,220 and $336 \mathrm{GHz}$. These peak values, except for $110 \mathrm{GHz}$, these values differ from the expected resonant frequencies. By changing the capacitance $C$ and the phase velocity $v_{p h}$, we can shift the positions of the peaks. However, we cannot obtain agreement for all three values for any choice of $C$ and $v_{p h}$. Also, the measured bandwidths of the high resonances are larger than expected. These discrepancies suggest that the loss and dispersion are not neglible at these frequencies. The admittance of transmission line with small loss and dispersion is given by ${ }^{38}$

$$
Y=\frac{Y_{o} \sin ^{2}[\beta(\omega) \ell]}{\alpha \ell}+i Y_{o} \tan [\beta(\omega) \ell] .
$$

Here $\alpha$ is the loss per unit length, and $\beta(\omega)=\omega / \mathrm{v}_{\mathrm{ph}}(\omega)$ is the dispersive propagation constant. If we assume the loss in the microstrip stub comes mainly from the surface impedance of the superconductor, then the frequency dependence of $v_{p h}$ can be written in the form

$$
\mathrm{v}_{\mathrm{ph}}=\frac{\mathrm{v}_{\mathrm{o}}}{1+\mathrm{a} \omega^{2}} \text {. }
$$

In Fig. 6.6c we replot the RF coupling coefficient $C_{R F}$ for a stub that is both lossy and dispersive. We chose $\alpha$ and a in Eqs. 6.7 and 6.8 to give a good fit to both resonant frequency and bandwidth for the three resonances. The values of the parameters obtained are $\alpha \ell / Y_{0}$ is $1.0 \Omega^{-1}$, and $a=3.8 \times 10^{-26}$ .The value of $\alpha$ deduced corresponds to a loss of $38 \mathrm{~dB}$ per meter. This is about 10 times more loss than is calculated using the Mattis-Bardeen 
theory ${ }^{78}$. By analyzing the shape of pumped I-V curves measured with the junction at $4.5 \mathrm{~K}$ and at $2.8 \mathrm{~K}$, we have determined that the loss is not temperature dependent, as would be expected if it were due to the surface impedance of the superconducting films. We therefore assign the loss either to the $\mathrm{SiO}$ dielectric layer in the microstrip line, or to excess residual resistance in the superconducting films.

The best mixer performance measured using this type of tuning at each frequency is summarized in the table below.

\section{Center Frequency}

90

180

270

360

Mixer Noise Temp.
$150 \mathrm{~K}$
$200 \mathrm{~K}$
$\sim 2000 \mathrm{~K}$
$>10,000 \mathrm{~K}$

\author{
Coupled Gain \\ $-4.8 \mathrm{~dB}$ \\ $-4.4 \mathrm{~dB}$ \\ $-10.2 \mathrm{~dB}$ \\ $<-15 \mathrm{~dB}$
}

In Fig. 6.7 we show a pumped I-V curve for a mixer with a stub designed for $180 \mathrm{GHz}$. The junction normal resistance is $64 \Omega$, and the leakage current is $3.5 \mu \mathrm{A}$ at $\mathrm{V}_{\mathrm{dc}}=2.5 \mathrm{mV}$. The first photon assisted tunneling step has a positive slope of about $200 \Omega$, which indicates that the capacitance of the junction is successfully resonated (see chapter 4 ).

In Fig. 6.8, we plot the receiver noise temperature, the mixer noise temperature, the available gain, and the coupled gain as function of LO frequency for a mixer with a stub designed for $180 \mathrm{GHz}$. The lowest mixer noise temperature is $200 \mathrm{~K}$ at $176 \mathrm{GHz}$, where the receiver noise temperature is $250 \mathrm{~K}$. The best coupled gain of $-4.4 \mathrm{~dB}$ occurs at the same frequency.

The pumped I-V curves of similar mixers designed for 270 and 360 $\mathrm{GHz}$ showed well developed photon-assisted tunneling steps. However, the mixer performance is very poor. The IF output power from the mixer is very low, only 2-3 times higher that the shot-noise background. This suggests that the high mixer noise temperature arises because of poor coupling of the $\mathrm{RF}$ signal to the mixer. One possibilty is that the loss in the stub, which should increase with frequency, is so large at these frequencies that it limits the performance of the tuning structures. This assumption is supported by the analysis of I-V curve shape, which indicates excess embedding conductance below the resonant frequency, which is expected for a lossy stub.

\subsection{2 $\lambda / 8$ Stub (Shorted-End Stub)}

This tuning element is similar to the open-ended stub, except that the end of the stub is RF shorted. This is achieved by a $\lambda / 4$ section of highadmittance (wide) microstrip line. A diagram of this configuration is shown in Fig. 6.9a. The high admittance of the line provides a good approximation to an RF short across a wide frequency band. The $3 \mathrm{~dB}$ linewidth of the resonance of this structure is expected to be about twice as large as the resonance associated with the open-ended stub. 
The total susceptance of the two-section stub is given by ${ }^{38}$

$$
B_{\text {stub }}(\omega)=\frac{Y_{1}\left[Y_{2} \tan \left(\beta \ell_{2}\right)+Y_{1} \tan \left(\beta \ell_{1}\right)\right]}{Y_{1}-Y_{2} \tan \left(\beta \ell_{1}\right) \tan \left(\beta \ell_{2}\right)}
$$

Where $\beta=\omega / v, Y_{1,2}$ are the characteristic admittances of section 1 (narrow) and section 2 (wide) of the stub. The resonance condition is given by

$$
\omega_{0} C+B_{\text {stub }}\left(\omega_{0}\right)=0 \text {. }
$$

We designed tuning structures of this type to operate at 90, 180, 270, and 360 GHz. The expected bandwidths of the tuning circuits are summarized below. All dimensions were optimized for maximum expected bandwidth. The junctions used for these mixers were fabricated using the tri-layer process at NIST in Boulder. These were prototype devices for which the processing was not yet optimized. These devices proved to be extremely sensitive to mounting-induced stress and were destroyed before definitive mixer tests could be parformed.

$\begin{array}{ll}\text { Center Frequency } & 3 \mathrm{~dB} \text { Bandwidth } \\ 90 & 10 \\ 180 & 5 \\ 270 & 4 \\ 360 & 4\end{array}$

$\mathrm{C}_{\mathrm{RF}}$ is plotted as a function of frequency in Fig $6.9 \mathrm{~b}$ for a tuning structure designed for $90 \mathrm{GHz}$. The junction capacitance and $\mathrm{G}_{\mathrm{RF}}$ are the same as in Fig. $6.6 \mathrm{~b}$. The junction response measured by the Fourier transform spectrometer is also shown in Fig. 6.9b.

\subsubsection{Five Junction Array with Inductive Wire}

Another type of tuning element is an inductive wire in parallel with a linear array of 5 junctions. A diagram of this configuration is shown in Fig. 6.10a. In order for this type of tuning element to function correctly, it must be in parallel with the junction array at the RF frequency, and an open circuit at $\mathrm{dc}$ and at the IF frequency. This is achieved by connecting one end of the inductive wire to a $\lambda / 4$ open-ended microstrip stub which provides an RF short at the design frequency. In this configuration, the inductance due to the junction leads is no longer negligible, and must be included in any calculation of $C_{R F}$. For large junctions with short leads, i.e. $G_{R F} \ll\left(C / L_{L}\right)^{1 / 2}$, the resonant frequency is given by

$$
\omega_{0}=\left[C\left(L_{W}+L_{L}\right)\right]^{1 / 2}
$$


The inductance due to the junction leads, together with the inductive wire, works as an impedance transformer and transforms $\mathrm{G}_{\mathrm{RF}}$ of the junction array to a higher value. Feldman ${ }^{60}$ has shown that an array of $n$ junctions can be treated as a single junction with $G_{R F}=n G_{R F}$, single junction and $C=(1 / n) C_{\text {single }}$ junction. The inductive wire cannot be used in the single junction case tecause of geometrical considerations. Above about $150 \mathrm{GHz}$, this tuning scheme becomes ineffective when using current fabrication schemes because of the series resonance between the inductance of the junction leads and the junction capacitance. Below this frequency, however, it has the advantage of a wider bandwidth than the either of the stub based tuning elements.

We fabricated a five junction array with an inductive wire tuning element designed to operate at $100 \mathrm{GHz}$. The inductive wire was $60 \mu \mathrm{m}$ long, $5 \mu \mathrm{m}$ wide and $0.27 \mu \mathrm{m}$ thick, giving a geometrical inductance of $44 \mathrm{pH}$. The inductance due to the junction leads is estimated to be about $34 \mathrm{pH}$. The estimated capacitance is $32 \mathrm{fF}$, giving a resonant frequency of $101 \mathrm{GHz}$. These two inductances worked together to transform up the junction admittance at the operating frequency by about a factor of 3 .

The response of the junction array, measured by the Fourier transform spectrometer is shown in $6.10 \mathrm{~b}$. Also shown is the best coupled mixer gain measured at several frequencies near the resonant peak. The coupled gain of the mixer is very highly correlated with the measured direct detector response, and hence with the RF coupling coefficient, as postulated earlier. The lowest noise temperature measured for this type of tuning structure is $115 \mathrm{~K}$, with a coupled gain of $-3.4 \mathrm{~dB}$.

\subsubsection{Open Ended Stub with $\lambda / 4$ Matching Transformer.}

The stub-type tuning structures discussed so far (sections 6.5.2 and 6.5.3) do not transform the real part of the junction admittance. At submillimeter frequencies, it becomes difficult to fabricate junctions with small enough values of $\omega R_{N} C$ (we want $\omega R_{N} C \sim 4$ ) with high enough values of $R_{N}$ to efficiently couple to typical antenna admittances. Small $\omega R_{N} C$ requires high critical current densities, which in turn requires extremely small junction areas to obtain high enough $R_{N}$ 's. The requirement of small junction area can be relaxed if the real part of the RF junction admittance can be transformed down to match the output admitiance of the antenna. This can be achieved using a $\lambda / 4$ matching transformer. ${ }^{82}$

An example of such a configuration is shown in Fig. 6.11a. In this configuration, the junction capacitance is resonated using a $3 \lambda / 8$ stub as in section 6.5.2. Then the relatively high input admittance of a high current density, moderate area junction is transformed down to match the lower output admittance at the antenna terminals.

We have designed tuning elements to match $2 \times 2 \mu \mathrm{m}$ trilayer ${ }^{50,83}$ junctions with a critical current density of $5 \mathrm{kA} / \mathrm{cm}^{2}$ to a $\log$-periodic antenna. These junctions have a normal resistance of $10 \Omega$, far too low to 
efficiently match to the $0.008 \Omega^{-1}$ output admittance of the log-periodic antenna. We chose the characteristic admittance of the $3 \lambda / 8$ stub to maximize the bandwidth over which the capacitance is resonated. We then chose the characteristic admittance of the $\lambda / 4$ transformer to maximize the frequency range in which $C_{R F}$ is greater than 0.8 , giving an admittance about $30 \%$ higher than the geometric mean of the RF input admittance of the junction and the output admittance of the antenna. We have designed tuning elements for operation at $90,180,270$ and $360 \mathrm{GHz}$. A summary of expected bandwidths is shown in the table below. In Fig 6.11b, we plot the expected $C_{R F}$ as a function of frequency for a tuning structure designed for $90 \mathrm{GHz}$. The expected bandwidths are much greater than those for the other tuning schemes discussed so far, due mostly to the smaller values of $\omega R_{N} \Gamma$ possible with this tuning scheme. The junctions and tuning structures are currently being fabricated at Conductus, and are not yet available for testing.

Center Frequency

90

180

270

360
Resonant Bandwidth

60 $\mathrm{GHz}$

$80 \mathrm{GHz}$

$85 \mathrm{GHz}$

$100 \mathrm{GHz}$

\subsection{Conclusion}

We have discussed the design and implementation of several types of tuning elements used to resonate the junction capacitance of millimeterwave and submillimeter-wave quasiopticaliy coupled SIS mixers. These tuning elements met with limited success at near millimeter wavelengths. As the operating frequency is increased into the submillimeter, the tuning schemes appear to be limited by loss in the superconducting transmission lines. Because this loss appeared to be independent of temperature, we blamed it either on dielectric losses in the interlayer dielectric used to form the transmission lines, or on excessive residual surface resistance of the superconducting films. Such losses probably become even more important at higher frequencies. Clearly, more work needs to be done in this area. 


\section{References}

1. J. R. Tucker, "Quantum Limited Detection in Tunnel Junction Mixers," IEEE J. Quantum Electron., vol. QE-15, pp. 1234-1258, 1979.

2. J. R. Tucker and M. J. Feldman, "Quantum Detection at Millimeter Wavelengths," Rev. Mod. Phys., vol. 57, pp. 1055-1113, 1985.

3. J. R. Tucker, "Predicted Conversion Gain in Superconductor-InsulatorSuperconductor Quasiparticle Mixers," Appl. Phys. Lett., vol. 36, pp. 477-479, 1980.

4. P. L. Richards and Q. Hu, "Superconducting Components for Infrared and Millimeter-Wave Receivers," Proc. IEEE, vol. 77, pp. 1233-1246, 1989.

5. N. R. Werthhamer, "Nonlinear Self-Coupling of Josephson Radiation in Superconducting Tunnel Junctions," Phys. Rev., vol. 147, pp. 255-263, 1966.

6. Q. Hu, C. A. Mears, P. L. Richards and F. L. Lloyd, "Observation of Nondissipative Quasiparticle Tunneling Currents in Superconducting Tunnel Junctions," Phys. Rev. Lett., vol. 64, pp. 2945-2948, 1990.

7. Q. Hu, C. A. Mears, P. L. Richards and F. L. Lloyd, "Quantum Susceptance and Its Effects on the High Frequency Response of Superconducting Tunnel Junctions," Phys. Rev. B, vol. 42, pp. 10250-10263, 1990.

8. C. A. Mears: Q. Hu and P. L. Richards, "Numerical Simulation on Experimental Data from Planar SIS Mixers with Integrated Tuning Elements," IEEE Trans. Magn., vol. MAG-25, pp. 1050-1053, 1989.

9. C. A. Mears, Q. Hu, P. L. Richards, A. H. Worsham, D. E. Prober and A. V. Raisanen, "Quantum Limited Quasiparticle Mixers at $100 \mathrm{GHz}$," IEEE Trans. Mag., vol. MAG-27, pp. 3363-3369, 1991.

10. C. A. Mears, Q. Hu, P. L. Richards, A. H. Worsham, D. E. Prober and A. V. Raisanen, "Quantum Limited Heterodyne Detection of Millimeter Waves Using Superconducting Tantalum Tunnel Junctions," Appl. Phys. Lett., vol. 57, pp. 2487-2489, 1990.

11. Q. Hu, C. A. Mears, P. L. Richards and F. L. Lloyd, "Millimeter Wave Quasioptical SIS Mixers," IEEE Trans. Magn., vol. MAG-25, pp. 1380-1383, 1989.

12. Tinkham, Introduction to Superconductivity. New York: McGraw-Hill, 1975.

13. J. Bardeen, L. N. Cooper and J. R. Schrieffer, "Theory of Superconductivity," Phys. Rev., vol. 108, pp. 1175-1204, 1957.

14. M. H. Cohen, L. M. Falicov and J. C. Phillips, "Superconductive Tunneling," Phys. Rev. Lett., vol. 8, pp. 316-318, 1962.

15. B. D. Josephson, "Possible New Effects in Superconductive Tunneling," Phys. Lett., vol. 1, pp. 251-253, 1961.

16. B. D. Josephson, "Coupled Superconductors," Rev. Mod. Phys., vol. 36, pp. 216-220, 1964.

17. J. Bardeen, "Tunneling from a Many-Body Point of View," Phys. Rev. Lett., vol. 6; pp 57-59, 1961 . 
18. V. Ambegaokar and A. Baratoff, "Tunneling Between Superconductors," Phys. Rev. Lett., vol. 10, pp. 486-489, 1963.

19. R. E. Harris, "Cosine and Other Terms in the Josephson Tunneling Current," Phys. Rev. B, vol. 10, pp. 84-94, 1974.

20. R. E. Harris, "Josephson Tunneling Current in the Presence of a Time Dependent Voltage," Phys. Rev. B, vol. 11, pp. 3329-3333, 1975.

21. R. E. Harris, "Intrinsic Response Time of a Josephson Tunnel Junction," Phys. Rev. B, vol. 13, pp. 3818-3821, 1976.

22. H. C. Torrey and C. A. Whitmer, Crystal Rectifiers. MIT Radiation Lab. Series New York: McGraw-Hill, 1948.

23. A. A. M. Saleh, Theory of Resistive Mixers. Res. MonoGraph 64 Cambridge: MIT Press, 1971.

24. M. J. Wengler and D. P. Woody, "Quantum Noise in Heterodyne Detection," IEEE Jour. Quantum Electron., vol. QE-23, pp. 613-622, 1987.

25. C. M. Caves, "Quantum Limit on Noise in Linear Amplifiers," Phys. Rev. $D$, vol. 26, pp. 1817-1839, 1981.

26. J. D. Jackson, Ciassical Electrodynamics. New York: John Wiley and Sons, 1975.

27. K. K. Likharev, Dynamics of Josephson Junctions and Circuits. New York: Gordon and Breach, 1986.

28. A. Barone and G. Paterno, Physics and Applications of the Josephson Effect. New York: John Wiley and Sons, 1982.

29. M. J. Feldman, "Some Analytical and Intuitive Results in the Quantum Theory of Mixing," J. Appl. Phys., vol. 53, pp. 584-592, 1982.

30. O. H. Soerensen, J. Mygind and N. F. Pedersen, "Measured Temperature Dependence of the Cosine Phi Conductivity in a Josephson Tunnel Junction," Phys. Rev. Lett., vol. 39, pp. 1018-1021, 1977.

31. C. A. Hamilton and S. Shapiro, "Experimental Demonstration of the Riedel Peak," Phys. Rev. Lett., vol. 26, pp. 426-428, 1971.

32. A. H. Dayem and R. J. Martin, "Quantum Interaction of Microwave Radiation with Tunneling Between Superconductors," Phys. Rev. Lett., vol. 8, pp. 246-248, 1962.

33. P. K. Tien and J. P. Gordon, "Multiphoton Processes Observed in the Interaction of Microwave Fields with the Tunneling Between Superconductor Films," Phys. Rev., vol. 129, pp. 647-651, 1963.

34. A. Yariv, Quantum Electronics. New York: John Wiley and Sons, 1975.

35. D. Rogovin and D. J. Scalapino, "Fluctuation Phenomena in Tunnel Junctions," Annals of Physics, vol. 86, pp. 1-90, 1974.

36. P. L. Richards, T.-M. Shen, R. E. Harris and F. L. Lloyd, "SIS Quasiparticle Junctions as Microwave Photon Detectors," Appl. Phys. Lett., vol. 36, pp. 777779, 1980.

37. G. S. Lee, "Superconductor-Insulator-Superconductor Reflection Parametric Amplifier," Appl. Phys. Lett., vol. 41, pp. 291-293, 1982.

38. R. E. Collin, Foundations for Microwave Engineering. New York: McGraw Hill, 1966. 
39. P. H. Siegel, "A Planar Log-Periodic Mixtenna for Millimeter and Submillimeter Wavelengths," IEEE Microwave Theory Tech., vol. MTT-S Digest, pp. 649-652, 1986.

40. R. C. Compton, R. C. McPhedran, Z. Popovic, G. M. Bebiez, P. P. Tong and D. B. Rutledge, "Bow-Tie Antennas on a Dielectric Half-Space: Theory and Experiment," IEEE Trans Ant. Prop, vol. AP-35, pp. 622-631, 1987.

41. M. N. Afsar, "Dielectric Measurements of mm-wave Materials," IEEE Trans. Microwave Theory Tech., vol. MTT-32, pp. 1598-1605, 1984.

42. Afsar, "Precision mm-Wave Dielectric Measurement of Birefringent Crystalline Sapphire and Ceramic Alumina," IEEE Trans. Instru. Meas., vol. IM-36, pp. 554-561, 1987.

43. W. H. Chang, "The Inductance of a Superconducting Strip Transmission Line," J. Appl. Phys, vol. 50, pp. 8129-8134, 1979.

44. J. H. Griener, C. J. Kircher and I. Ames, "Fabricating Process for Josephson Integrated Circuits," IBM J. Res. Dev., vol. 24, pp. 195-205, 1980.

45. A. R. Kerr, S. K. Pan and M. J. Feldman, "Integrated Tuning Elements for SIS Mixers," Int. J. Infrared and Millimeter Waves, vol. 9, pp. 203-212, 1988. 46. Q. Hu, C. A. Mears, P. L. Richards and F. L. Lloyd, "Measurement of Integrated Tuning Elements for SIS Mixers with a Fourier Transform Spectrometer," Int. Jour. Infrared and Millimeter Waves, vol. 9, pp. 303-320, 1988.

47. P. L. Richards and T. M. Shen, "Superconducting Devices for Millimeter Wave Detection, Mixing and Amplification," IEEE Trans. Electron. Dev., vol. ED-27, pp. 1909-1920, 1980.

48. J. E. Carlstrom, R. L. Planbeck and D. D. Thorton, "A Continuously Tunable 65-115 GHz Gunn Oscillator," IEEE Trans. Microwave Theory Tech., vol. MTT-33, pp. 610-619, 1985.

49. R. J. Bell, Introductory Fourier Transform Spectroscopy. New York: Academic Press, 1972.

50. M. Gurvitch, M. A. Washington and H. A. Huggins, "High Quality Refractory Josephson Junctions Utilizing Thin Aluminum Layers," Appl. Phys. Lett., vol. 42, pp. 472-474, 1983.

51. A. W. Lichtenberger, C. P. McClay, R. J. Mattauch, M. J. Feldman, S. K. Pan and A. R. Kerr, "Fabrication of $\mathrm{Nb} / \mathrm{Al}-\mathrm{Al}_{2} \mathrm{O}_{3} / \mathrm{Nb}$ Junctions with Extremely Low Leakage Currents," IEEE Trans. Magn., vol. MAG-25, pp. 1247-1250, 1989.

52. A. H. Worsham and D. E. Prober, "Quantum Tunneling Currents in a Superconducting Junction," Submitted to Physical Review Letters, vol. 1991. 53. P. L. Richards and S. A. Sterling, "Regenerative Josephson Effect Detector for Far Infrared Radiation," Appl. Phys. Lett., vol. 14, pp. 394-396, 1969.

54. P. Siedel and J. Richter, "Theoretical Invstigation of the Current-Voltage Characteristics of Superconducting Niobium-Lead Tunnel Junctions," Phys. Stat. Sol. A, vol. 98, pp. 189-197, 1980. 
55. A. D. Smith, W. R. McGrath, P. L. Richards, H. v. Kempen, D. E. Prober and P. Santhanam, "Negative Resistance and Conversion Gain in SIS Mixers," Physica B+C, vol. 108, pp. 1367-1386, 1981.

56. A. D. Smith and P. L. Richards, "Analytic Solutions to SuperconductorInsulator-Superconductor Quantum Mixer Theory," J. Appl. Phys., vol. 53, pp. 3806-3812, 1982.

57. T.-M. Shen and P. L. Richards, "Computer Simulations of the Performance of Quasiparticle Heterodyne Mixers," IEEE Trans. Magn., vol. MAG-17, pp. 677-683, 1981.

58. A. Skalare, "Determining Embedding Circuit Parameters from DC Measurements on Quasiparticle Mixers," Int. J. Infrared and Millimeter Waves, vol. 10, pp. 1339-1353, 1989.

59. A. V. Raisanen, W. R. McGrath, D. G. Crete and P. L. Richards, "Scaled Model Measurements of Embedding Impedances for SIS Waveguide Mixers," Int. J. Infrared and Millimeter Waves, vol. 6, pp. 1169-1189, 1985.

60. M. J. Feldman, S. K. Pan, A. R. Kerr and A. Davidson, "SIS Mixer Analysis Using a Scale Model," IEEE Trans. Magn., vol. MAG-19, pp. 494-497, 1983. 61. W. R. McGrath, P. L. Richards, D. W. Face, D. E. Prober and F. L. Lloyd, "Accurate Experimental and Theoretical Comparisons Between Superconductor-Insulator-Superconductor Mixers Showing Weak and Strong Quantum Effects," J. Appl. Phys., vol. 63, pp. 2479-2491, 1988.

62. W. R. McGrath, A. V. Raisanen and P. L. Richards, "Variable Temperature Loads for Use in Accurate Noise Measurements of Cryogenically Cooled Microwave Amplifiers and Mixers," Int. J. Infrared and Millimeter Waves, vol. 7, pp. 543-553, 1986.

63. D. W. Face, D. E. Prober, W. R. McGrath and P. L. Richards, "High Quality Tantalum Superconducting Junctions for Microwave Mixing in the Quantum Limit," Applied Physics Letters, vol. 48, pp. 1098-1100, 1986.

64. G.-J. Cui, D. W. Face, E. K. Trak, D. E. Prober, A. V. Raisanen, D. G. Crete and P. L. Richards, "High Quality Ta/PbBi Tunnel Junctions for 85-110 GHz Mixer Experiments," IEEE Trans. Magn., vol. MAG-23, pp. 688-691, 1987. 65. A. V. Raisanen, D. G. Crete, P. L. Richards and F. L. Lloyd, "Wide-Band Low-Noise Millimeter Wave SIS Mixers with a Single Tuning Element," Int. J. Infrared and Millimeter Waves, vol. 7, pp. 1835-1851, 1986.

66. T.-M. Shen, "Conversion Gain in Millimeter-Wave Quasiparticle Heterodyne Mixers," IEEE J. Quantum Electron., vol. QE-17, pp. 1151-1165, 1981.

67. T. G. Phillips and G. J. Dolan, "SIS Mixers," Physica, vol. 109 and 110B, pp. 2010-2019, 1982.

68. A. V. Raisanen, D. G. Crete, P. L. Richards and F. L. Lloyd, "A $100 \mathrm{GHz}$ SIS Quasiparticle Mixers with 10dB Coupled Gain," IEEE MTT-S Digest, vol. pp. 929-930, 1987.

69. M. J. Feldman, "Theoretical Considerations for THz SIS Mixers," Int. J. Infrared and Millimeter Waves, vol. 8, pp. 1287-1292, 1987. 
70. W. C. Danchi and E. C. Sutton, "Frequency Dependence of Quasiparticle Mixers," submitted to Journal of Applied Physics.

71. E. C. Sutton, W. C. Danchi, P. A. Jaminet and R. H. Ono, "A

Superconducting Tunnel Junction Receiver for $345 \mathrm{GHz}$," Int. J. Infrared and Millimeter Waves, vol. 11, pp. 133-150, 1990.

72. W. R. McGrath, personal communication, 1991.

73. M. J. Wengler, D. P. Woody, R. E. Miller and T. G. Phillips, "A Low-Noise Receiver for Millimeter and Submillimeter Wavelengths," Int. J. Infrared and Millimeier Waves, vol. 6, pp. 697-706, 1985.

74. L. Xizhi, P. L. Richards and F. L. Lloyd, "SIS Quasiparticle Mixers with Bowtie Antennas," Int J. Infrared and Millimeter Waves, vol. 9, pp. 101-133, 1988.

75. T. H. Buttgenbach, R. E. Miller, M. J. Wengler, D. M. Watson and T. G. Phillips, "A Broad-band Low-Noise SIS Receiver for Submillimeter Astronomy," IEEE Trans. Microwave Theory Tech., vol. MT-36, pp. 1720$1725,1988$.

76. Eccosorb CV-3, Emerson and Cumming, Canton, Massachusetts 02021.

77. Booker, "Slot Aerials and Their Relation to Complementary Wire Aerials," Journal of the IEE, vol. pt. III-A, pp. 620-627, 1946.

78. R. L. Kautz, "Miniaturization of Normal-State and Superconducting Striplines," Journal of Research of the National Bureau of Standards, vol. 84, pp. 247-259, 1978.

79. A. V. Raisanen, W. R. McGrath, P. L. Richards and F. L. Lloyd, "BroadBand RF Match to a Millimeter-Wave SIS Quasiparticle Mixer," IEEE Trans. Microwave Theory Tech., vol. MTT-33, pp. 1495, 1985.

80. M. D. Fiske, "Temperature and Magnetic Field Dependence of the Josephson Tunneling Current," Reveiws of Modern Physics, vol. 36, pp. 221, 1964.

81. A. V. Raisanen, W. R. McGrath, P. L. Richards and F. L. Lloyd, "BroadBand RF Match to a Millimeter-Wave SIS Quasiparticle Mixer," IEEE Trans. Microwave Theory Tech., vol. MTT-33, pp. 1495-1500, 1985.

82. J. A. Carpenter, A. D. Smith and E. R. Arambula, "100 GHz Mixer with Improved RF Matching," IEEE Trans. Magn., vol. MAG-27, pp. 2654-2657, 1991.

83. A. T. Barfknecht, R. C. Ruby and H. Ko, "A Simple and Robust Niobium Josephson Junction Integrated Circuit Process," IEEE Trans. Magn., vol. MAG-27, pp. 3125-3128, 1991. 


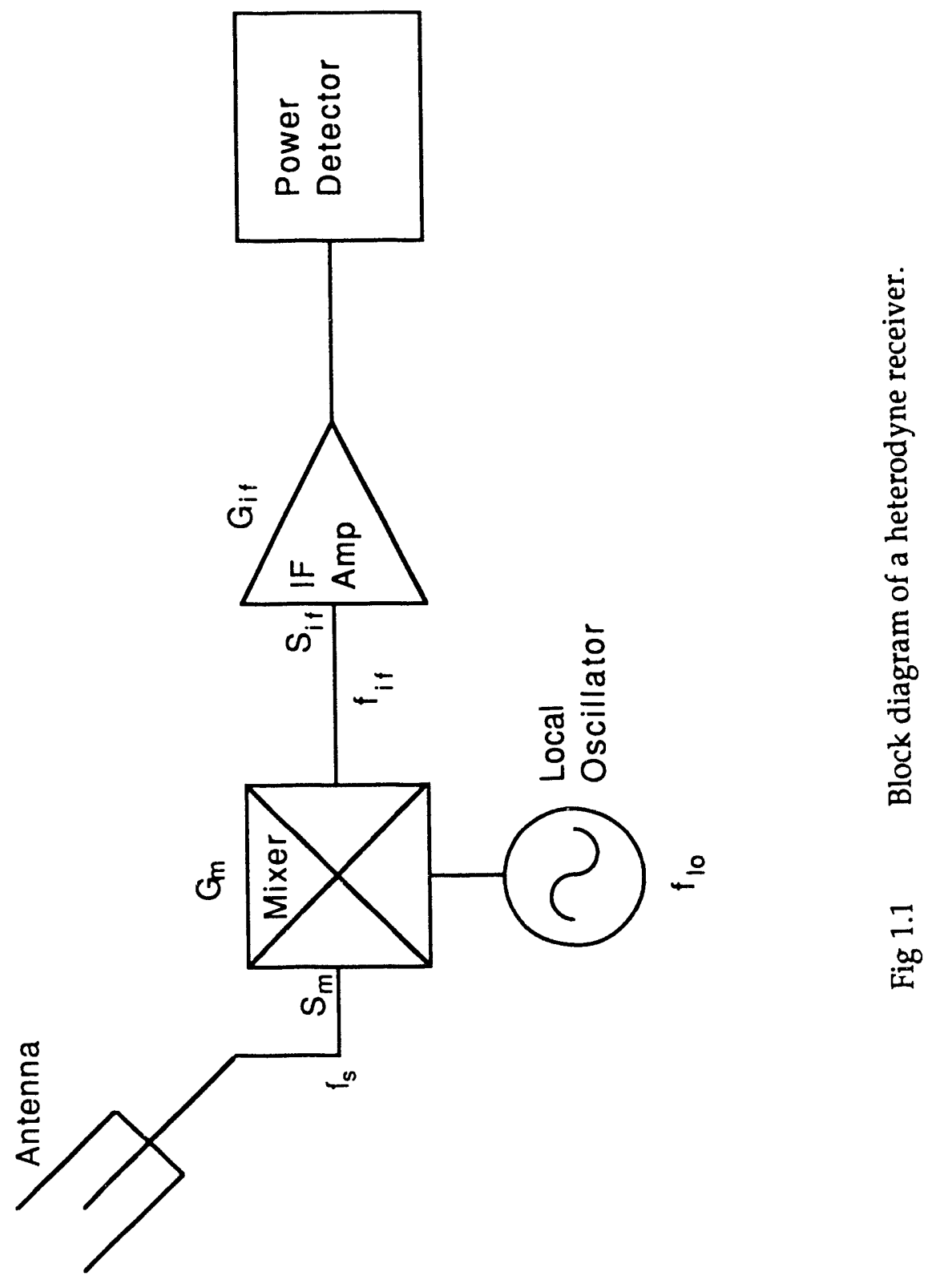



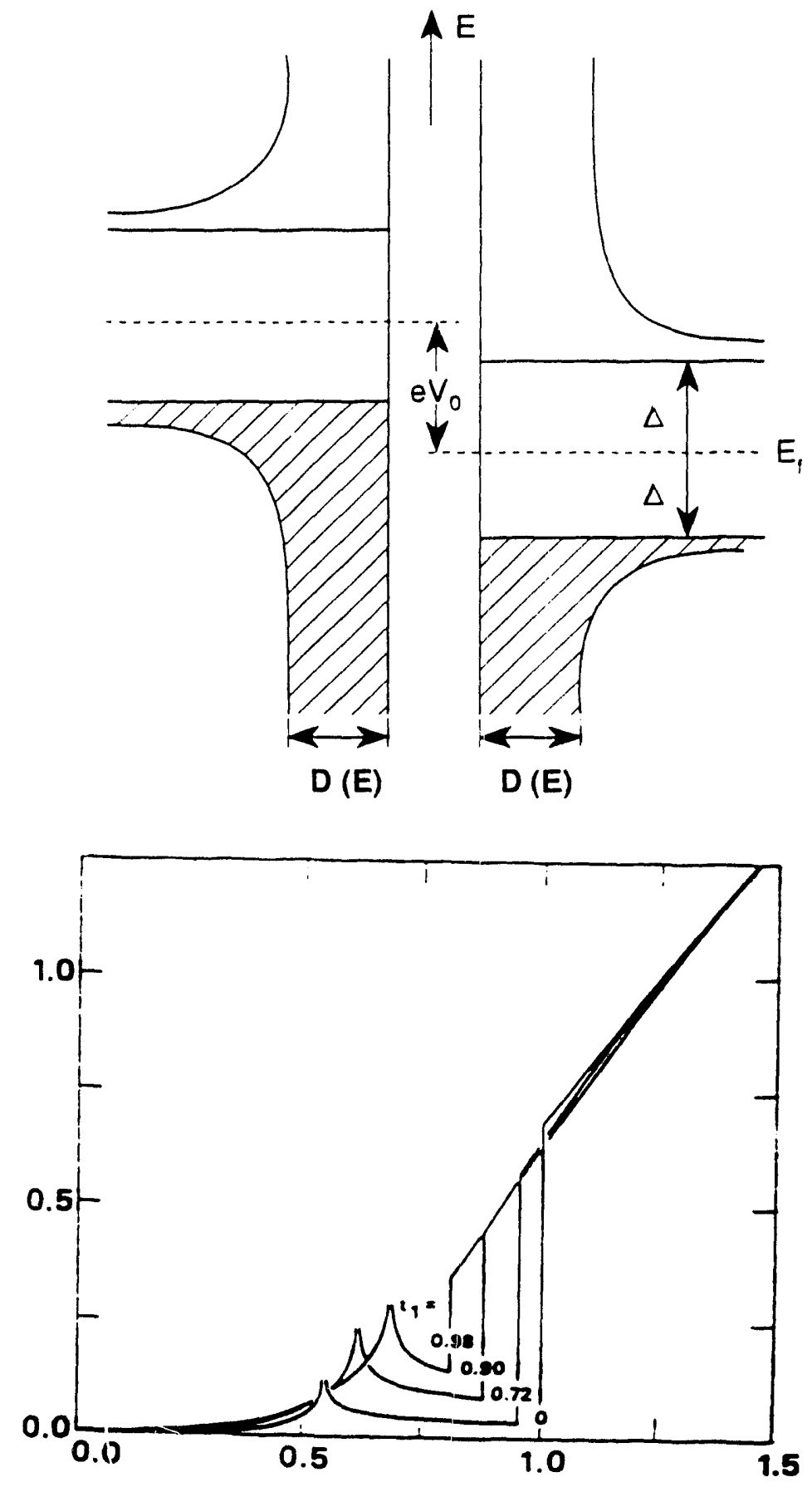

Fig. 2.1

a) Density of states

b) I-V curves as a function of temperature. 


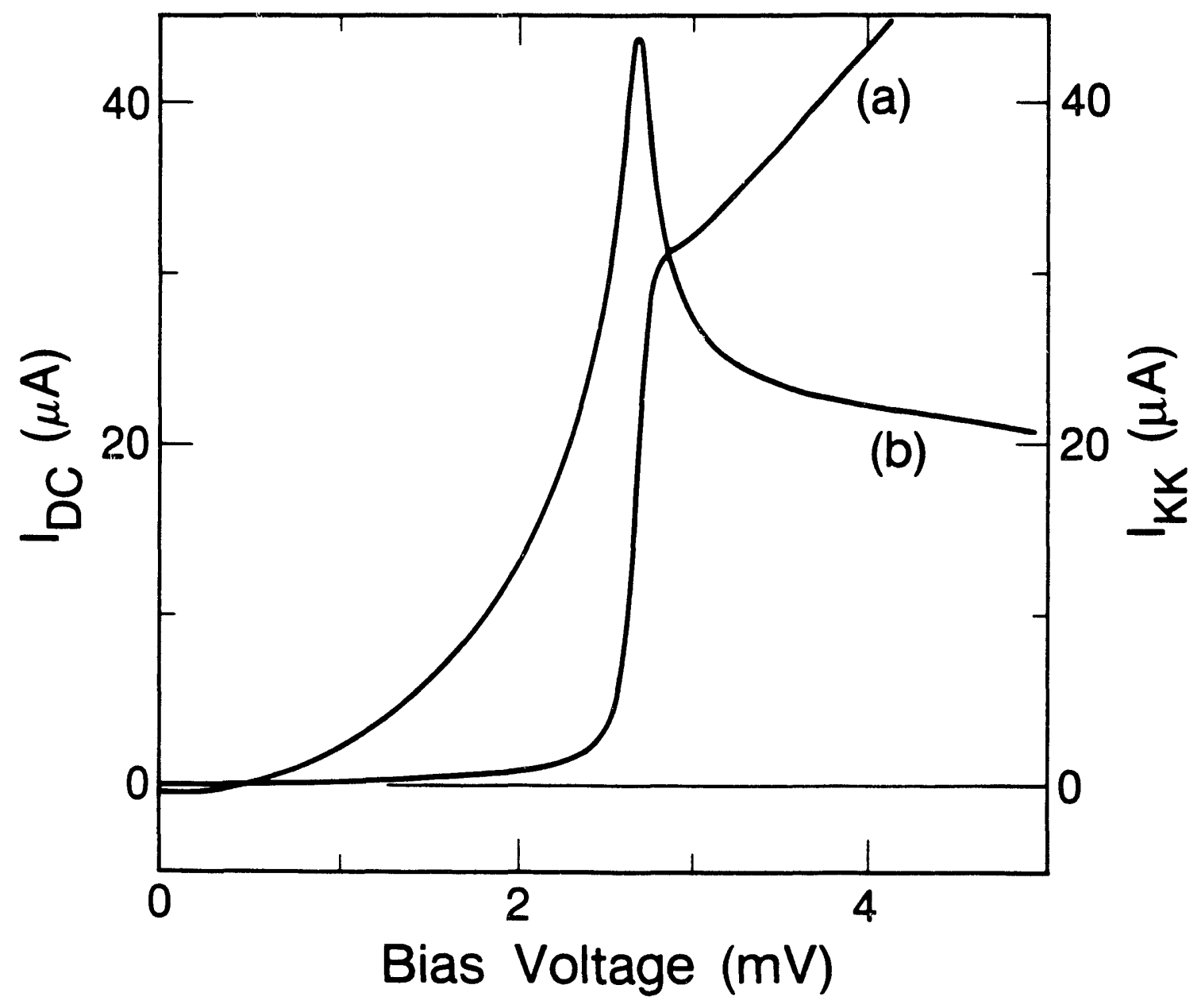

Fig. 2.2 Experimentally measured I-V curve, b) KK transform. 


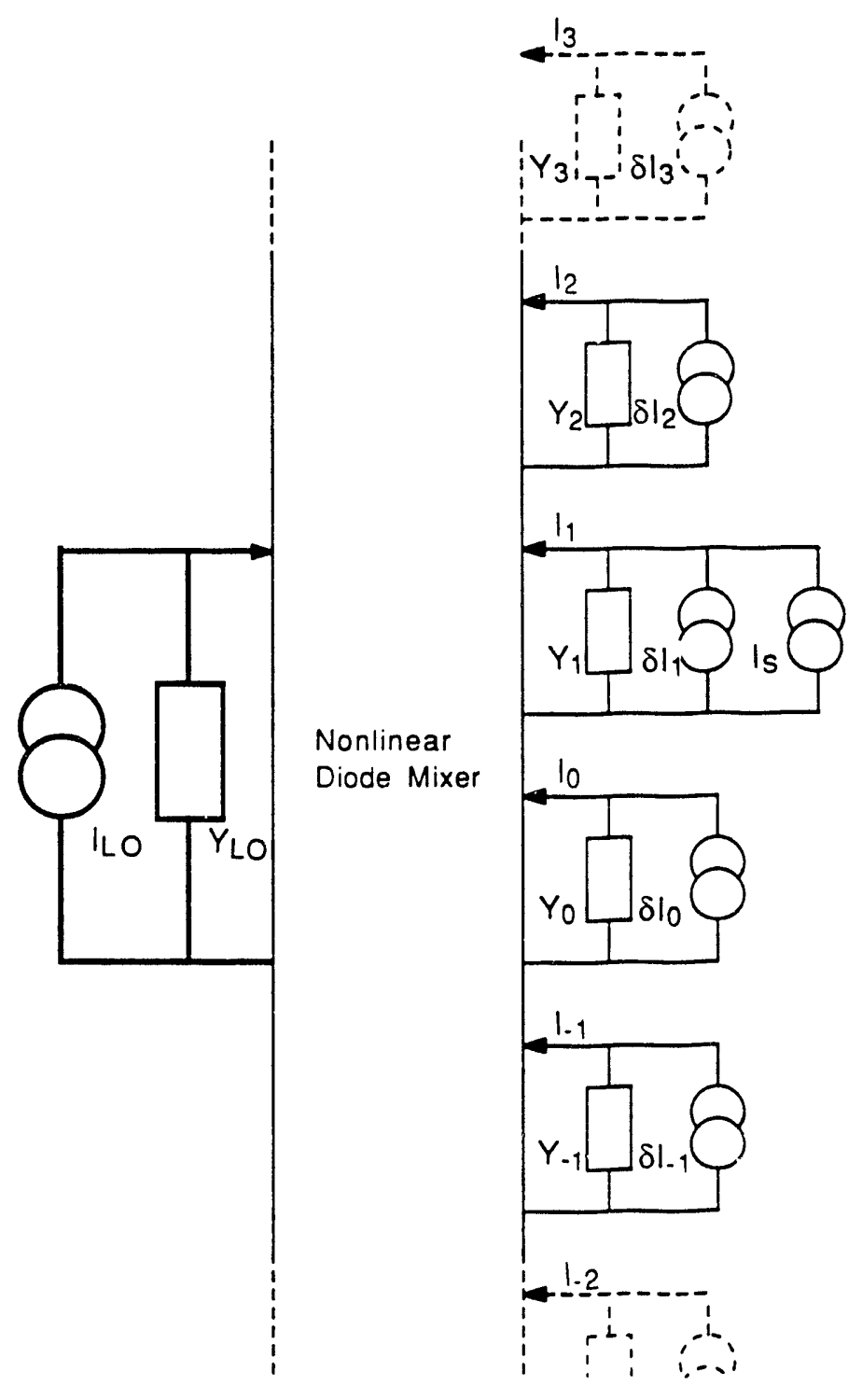

Fig. 2.3 Equivalent circuit used to analyze mixer performance. The voltage $v_{m}$ at the $m$ th port $\left(\omega_{m}=m \omega_{L O}+\omega_{I F}\right)$ is related to the currents $i_{n}$ at all other ports through the matrix $Y_{m n}$. Each port is terminated by an embedding admittance $Y_{m} \cdot A$ fluctuating current source $\delta I_{m}$ is placed at each port to account for noise. In typical operation, a signal source is placed at port 1 , and the IF output is measured at port 0 . In the three-port model, ports other than $-1,0$, and 1 are ignored. 


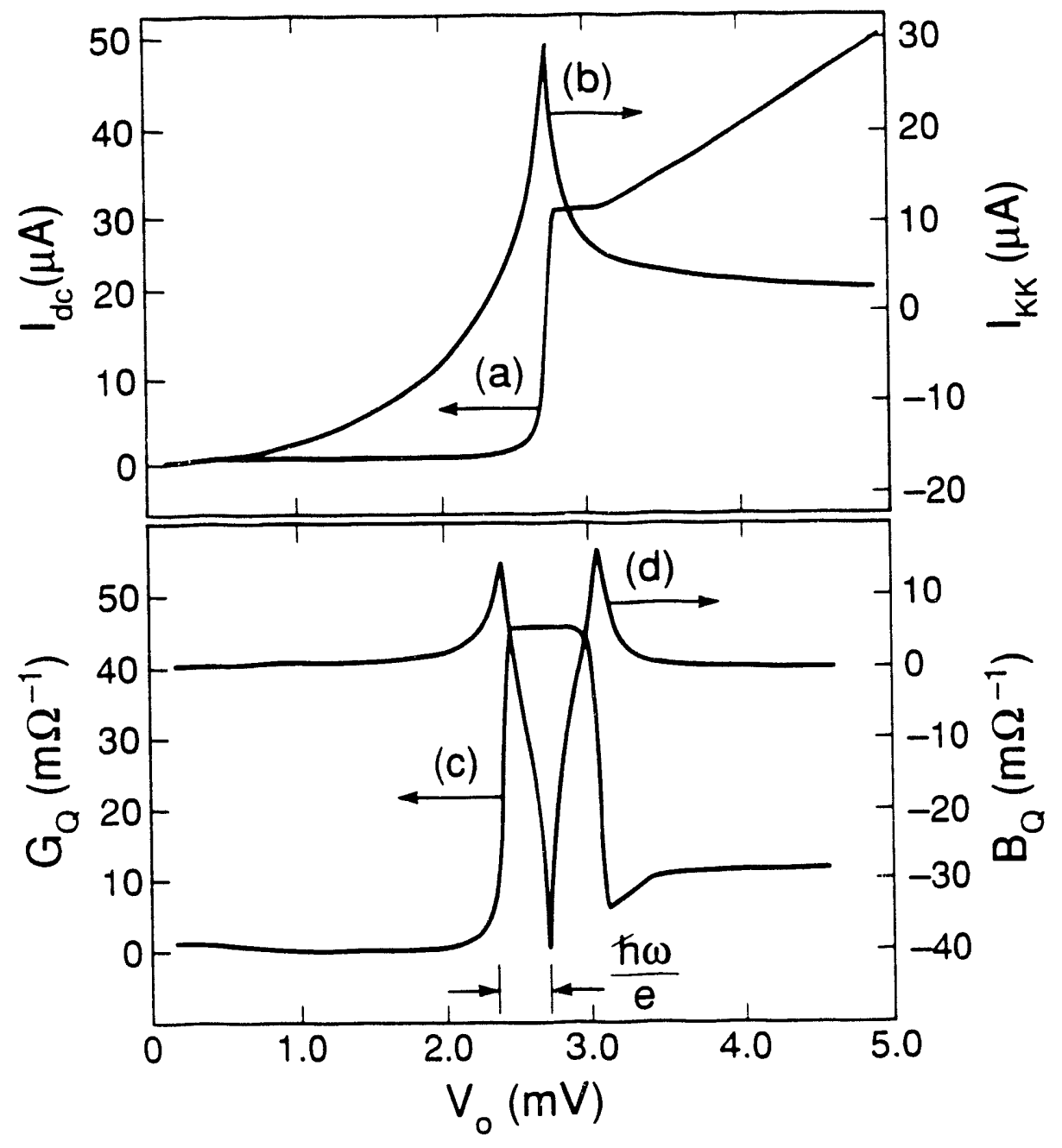

Fig. 3.1 a) Measured dc I-V curve of the SIS junction studied in this chapter, the junction temperature is about $4.5 \mathrm{~K}$. b) KramersKronig transform of the $I_{d c}$ calculated using Eq. 3.1. c) Quantum conductance $G_{4}$ at $\omega / 2 \pi=77 \mathrm{GHz}$ calculated from Eq. 3.5a using the $I_{d c}$ a). d) Quantum susceptance $B_{q}$ at 77 $\mathrm{GHz}$ calculated from Eq. $3.5 \mathrm{~b}$ using the $\mathrm{I}_{\mathrm{kk}}$ in $\mathrm{b}$ ). 


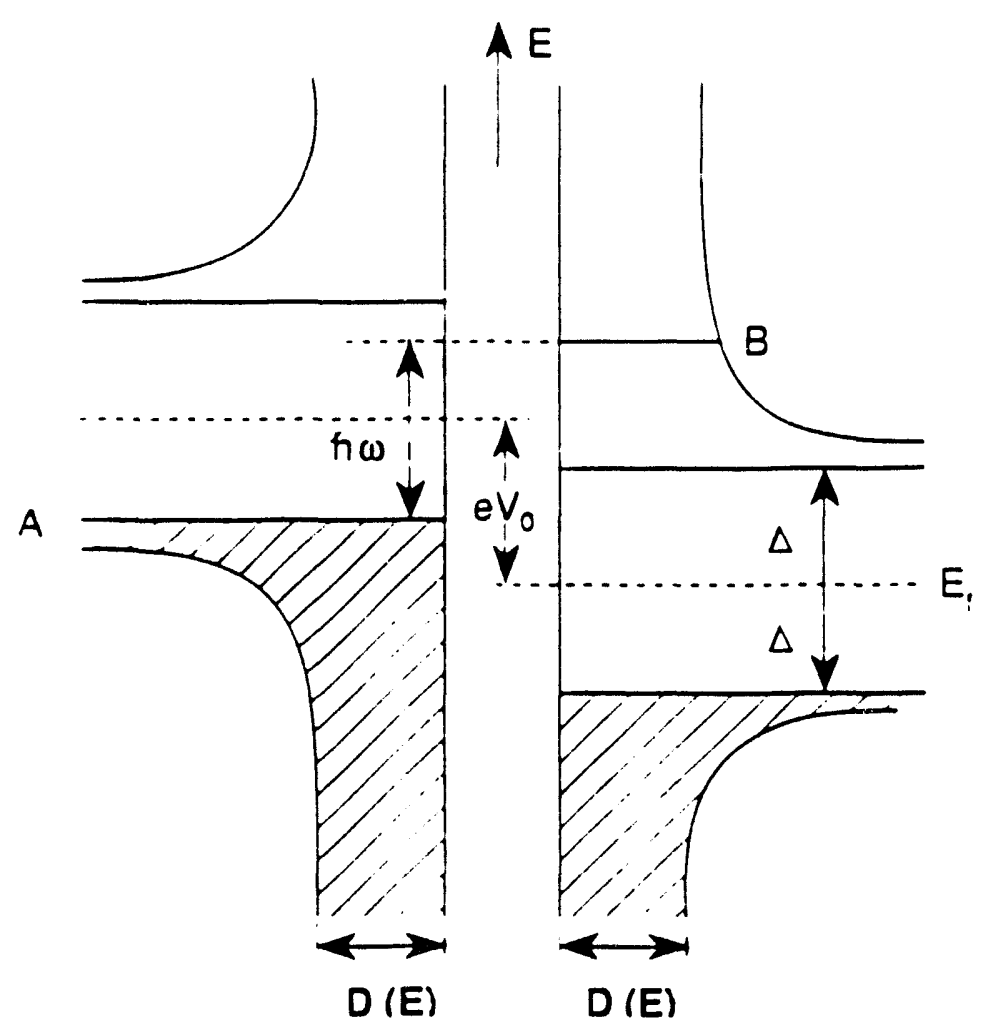

Fig. 3.2 Semiconductor model of an SIS junction. The energy difference between state $A$ on the left side and state $B$ on the right side is $\hbar \omega$, where $\omega$ is the angular frequency of the RF drive. The tunneling between states $A$ and $B$ can be assisted by the real photons in the RF signal. The tunneling between state $A$ and any states other than $B$ cannot be finalized because of the energy conservation law. The quantum sloshing between state $A$ and the states on the right side other than state $B$ gives a reactive component in the tunneling current. 


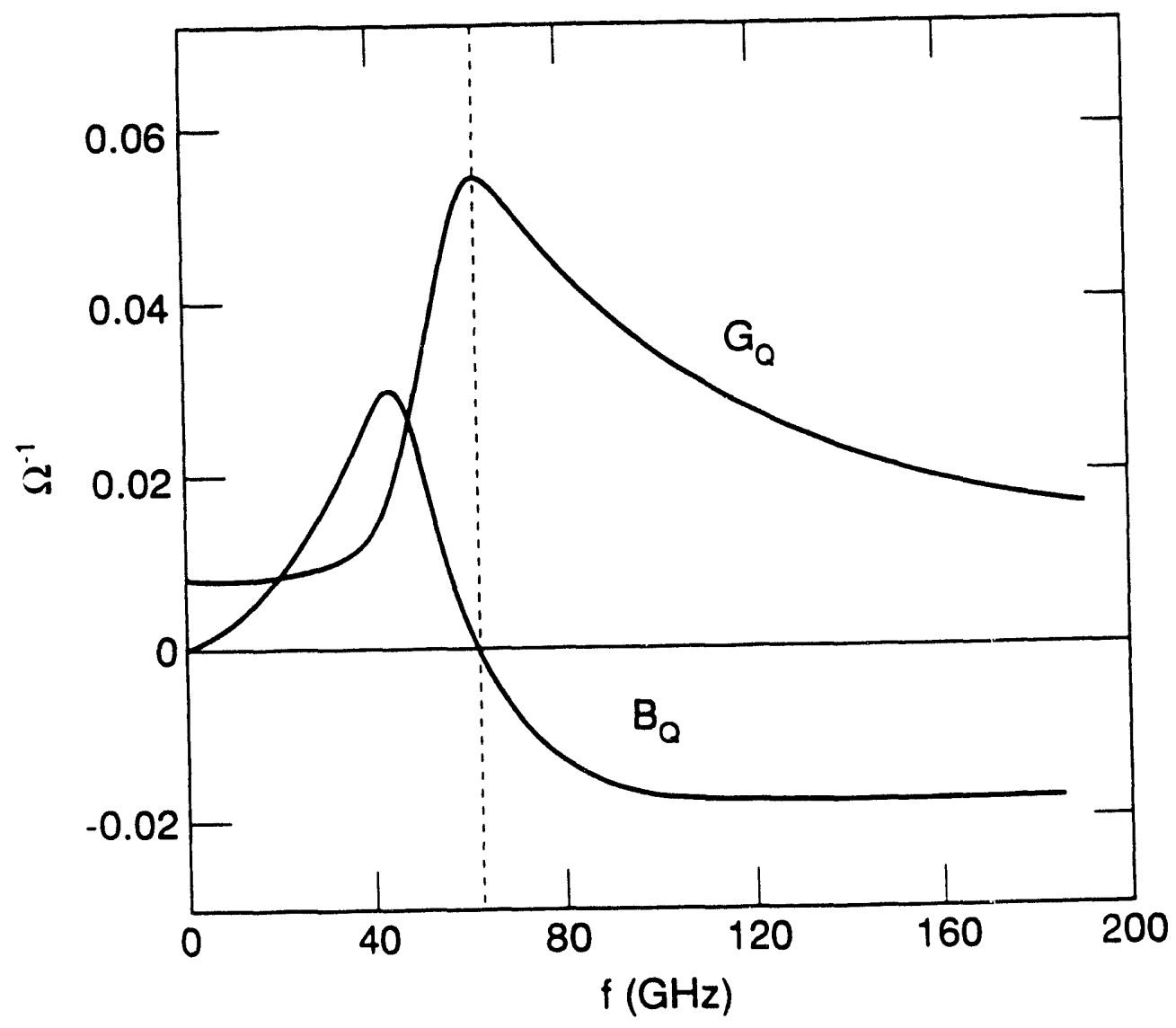

Fig. 3.3 Calculated quantum conductance $G_{q}$ and quantum susceptance $\mathrm{B}_{\mathrm{q}}$ using Eq. 3.5 as functions of frequency at a dc bias voltage $\mathrm{V}_{\mathrm{O}}=$ $2.50 \mathrm{mV}$. The frequency at the peak of $\mathrm{G}_{\mathrm{q}}, 62 \mathrm{GHz}$, corresponds to the energy difference between the edges of the valence band and of the conduction band on two sides of the junction. At this frequency, the quantum susceptance $B_{q}$ vanishes. 
Fig. 3.4 (On next page) a) Picture of a log-periodic antenna with a microstrip stub, the SIS junction is located at one end of the stub and at the center of the antenna. b) Schematic of a two-secticn microstrip stub with one section RF short-ended by the other section, which is a $\lambda / 4$ long, open-ended stub. c) Equivalent circuit of a resonator which includes a microstrip stub $B_{\text {stub }}, a$ junction capacitance $C$, the quantum susceptance $B_{q}$, and the quantum conductance $G_{q}$. The variable signs on $B_{q}$ and $G_{q}$ indicate that they are functions of $\mathrm{dc}$ bias voltage. The radiation source and the antenna are represented with an RF current source in parallel with the antenna admittance $\mathrm{Y}_{\mathrm{A}}$. 


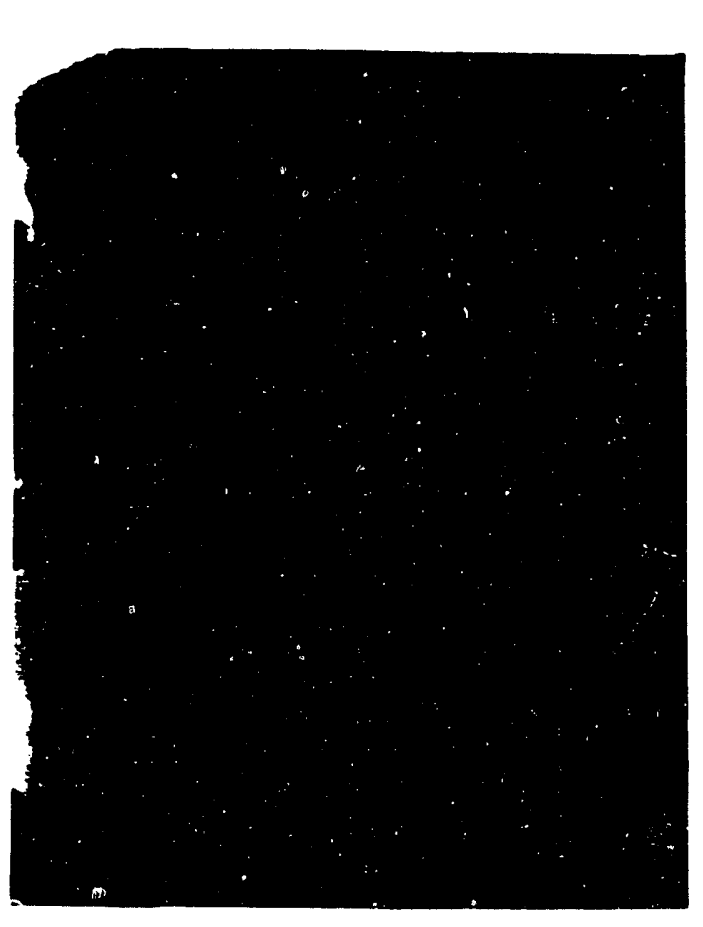

(a)

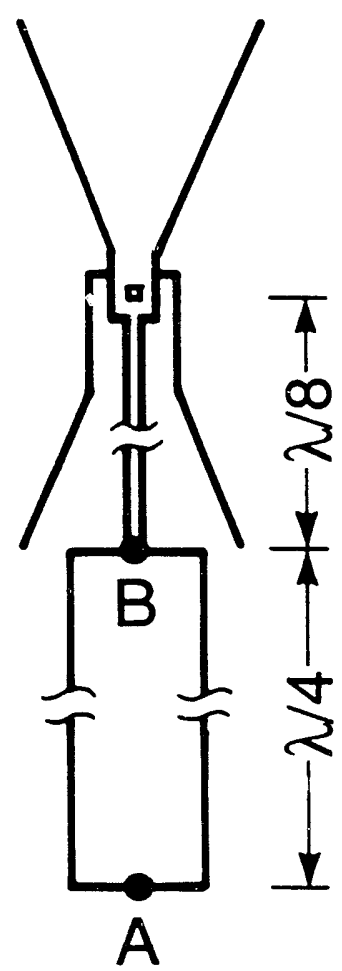

(b)

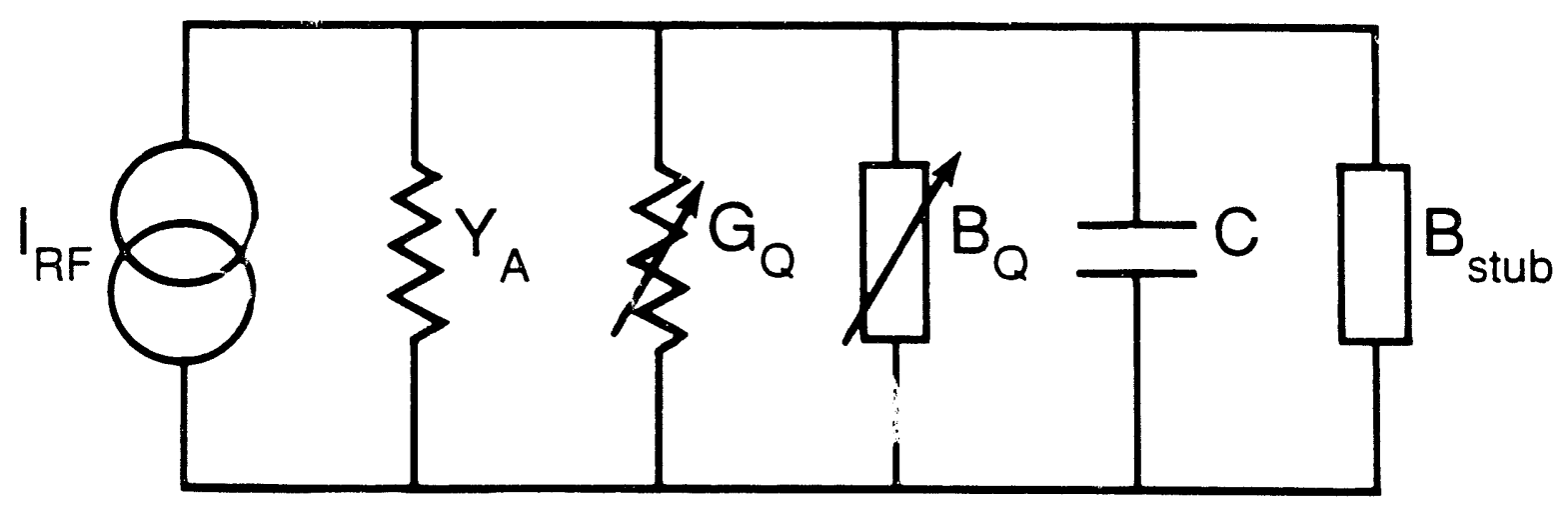



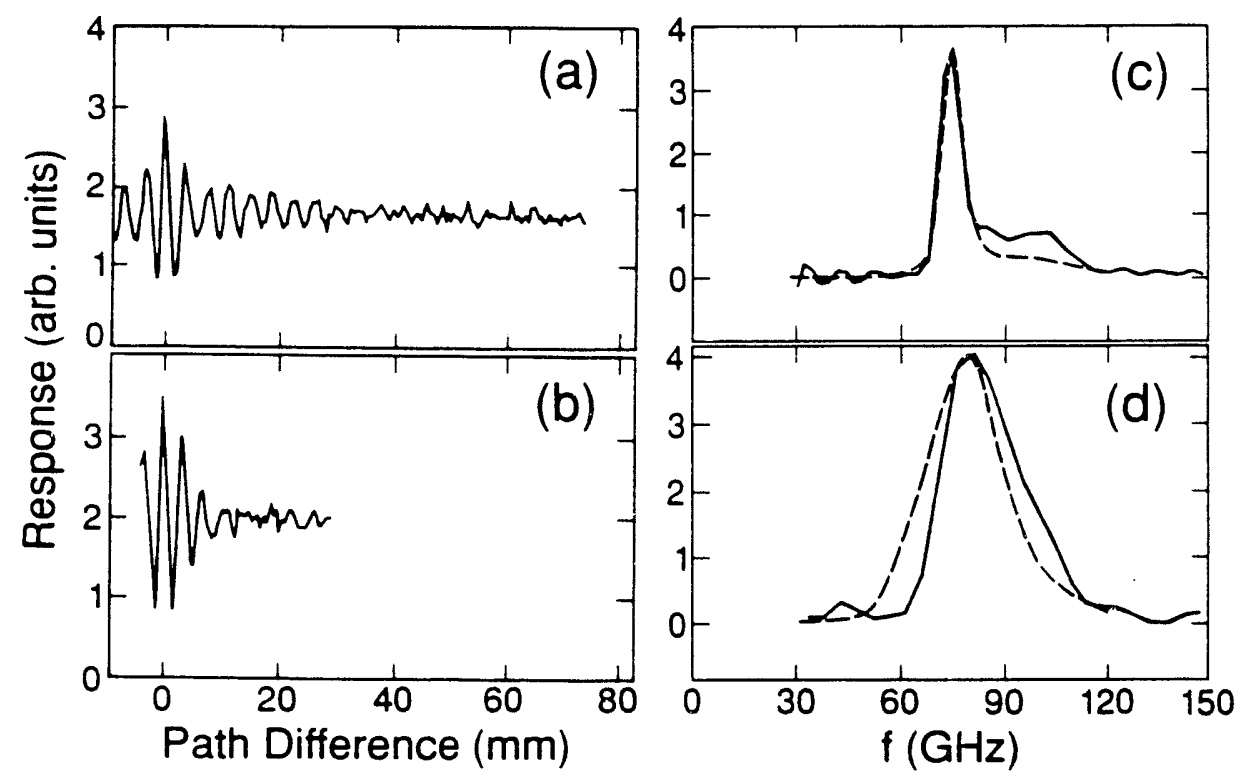

Fig. 3.5 Interferograms taken with a Fourier transform spectrometer, a) at $2.35 \mathrm{mV}, \mathrm{b})$ at $2.50 \mathrm{mV}$. Spectra after correcting for beamsplitter efficiency corresponding to the above interferograms, c) at 2.35 $\mathrm{mV}, \mathrm{d}$ ) at $2.50 \mathrm{mV}$. The dashed lines in c) and d) are the computied spectra. 


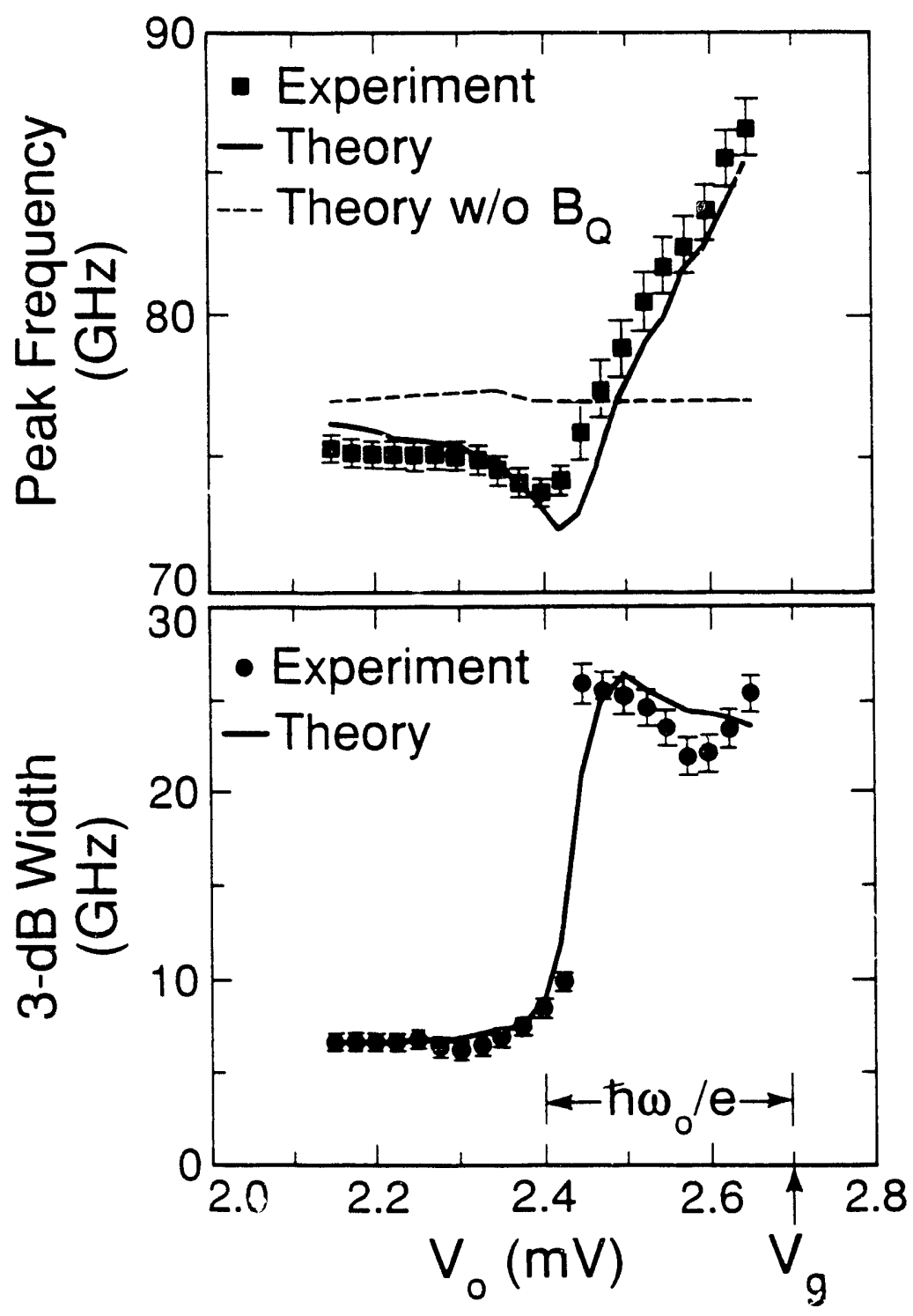

Fig. 3.6 a) Resonant frequency as a function of dc bias voltage. The dots are the experimentally measured results; the solid line is the theoretically calculated results; the dashed line is the calculated result without including the quantum susceptance. Note the dashed line is essentially flat vs. $V_{0}$. b) Linewidth of the resonance as a function of $\mathrm{V}_{\mathrm{o}}$. The dots are the experimental results and the solid line is the calculated result. $\mathrm{w}_{0} / 2 \pi=77 \mathrm{GHz}$ is the resonant frequency of the imbedding susceptance without the quantum susceptance. 


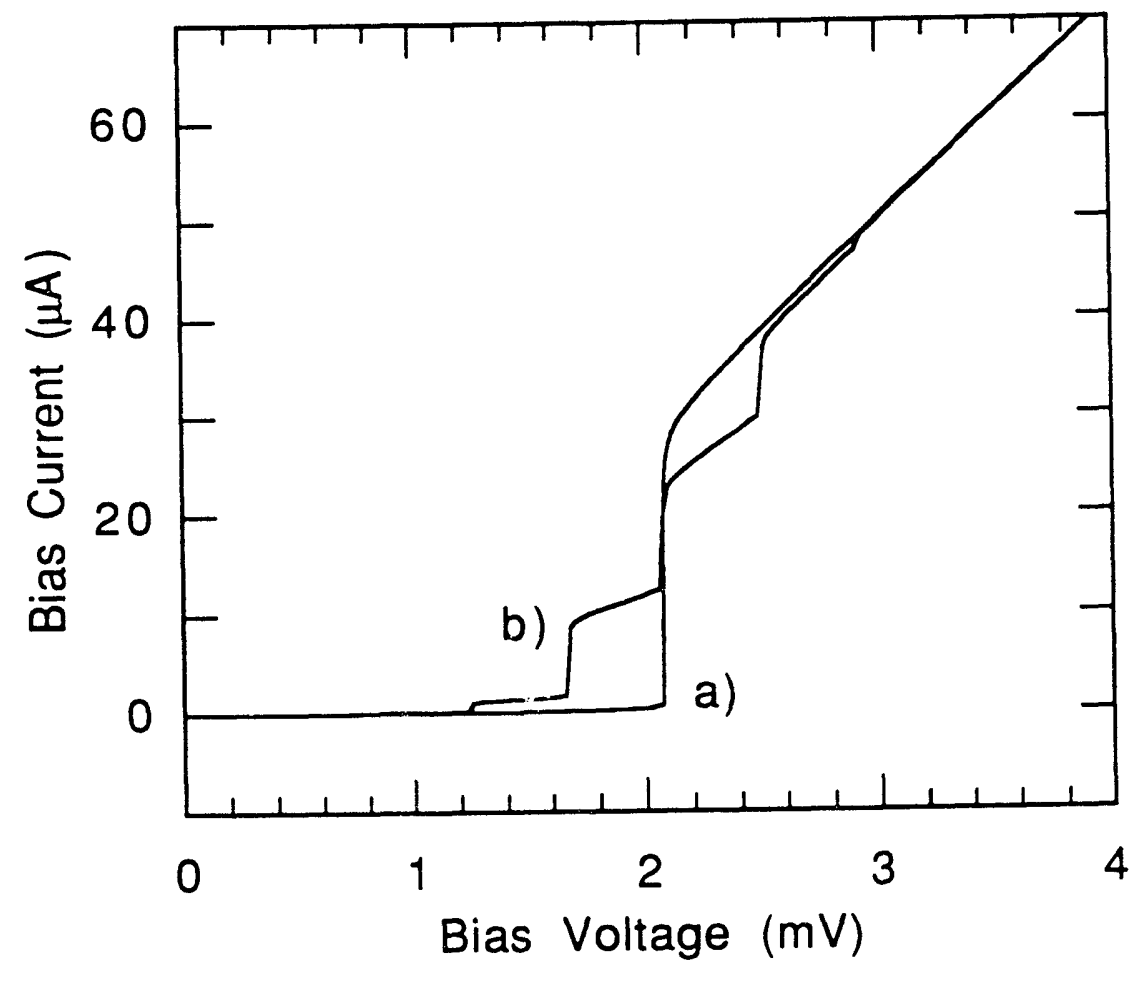

Fig. 4.1 a) Experimentally measured dc I-V curve of an SIS tunnel junction. b) Voltage-pumped I-V curve calculated using the dc $\mathrm{I}-\mathrm{V}$ curve shown in a). The reduced pump voltage $\alpha=\mathrm{eV}_{\omega} / \hbar \omega$ used is 1.3 , and the pump frequency used is $100 \mathrm{GHz}$. 


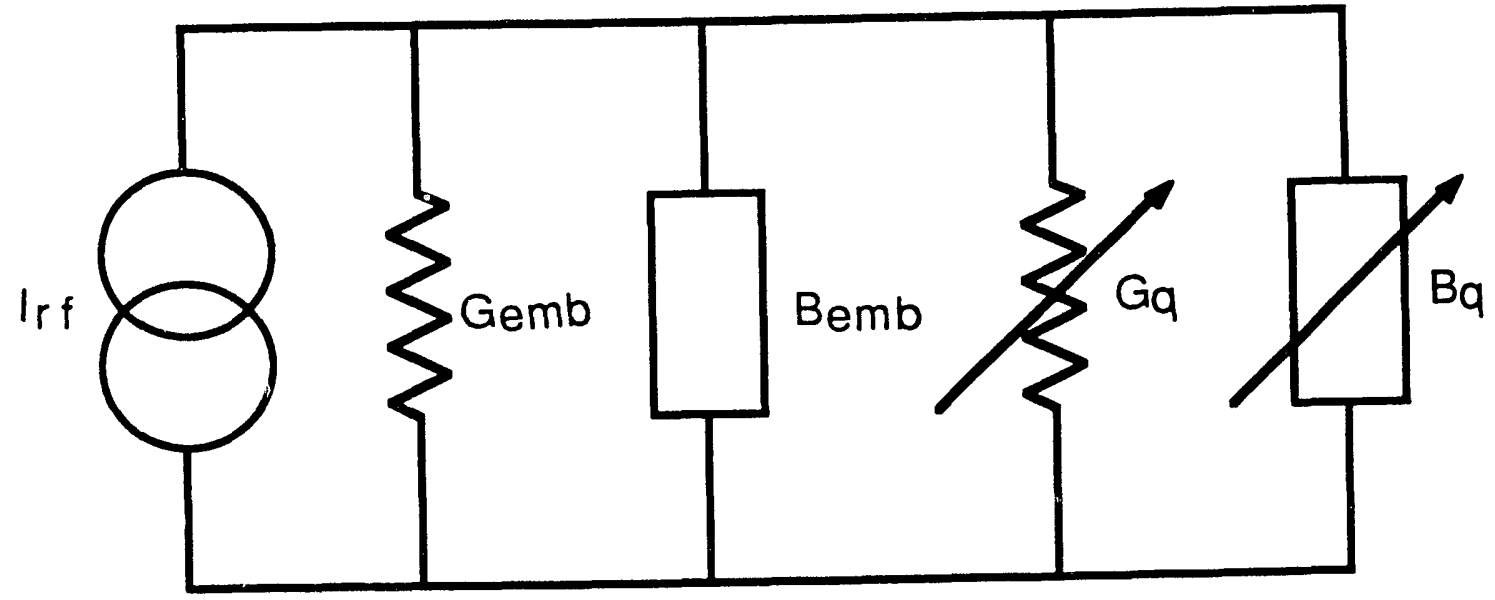

Fig. 4.2 Equivalent circuit used for the analysis of pumped I-V curves. The embedding admittance $Y_{e m b}=G_{e m b}+i B_{e m b}$ is the parallel combination of the susceptance due to the geometrical capacitance of the junction and the admittance due to the mixer mount. The quantum admittance $\mathrm{Y}_{\mathrm{q}}=\mathrm{G}_{\mathrm{q}}+\mathrm{iB}_{\mathrm{q}}$ is due only to the tunneling currents in the device. 


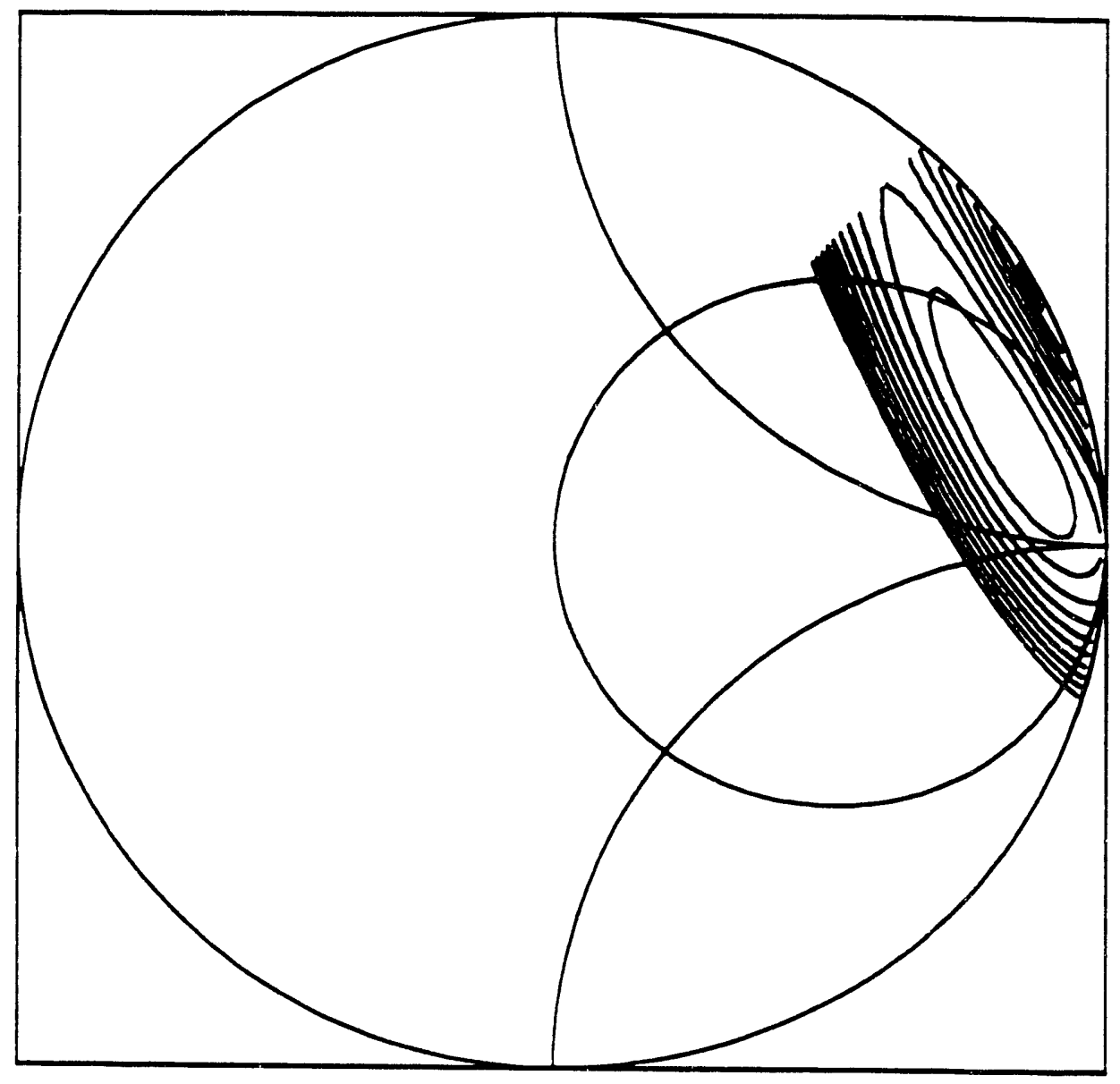

Fig. 4.4 Example of a contour map of the fit parameter $\epsilon$ plotted as a function of embedding admittance on a admittance Smith chart. Contour spacing is $10^{-5} \mathrm{mV}^{2}$. The quality of the fit improves as the admittance move toward the center of the series of concentric oblong contours. When a pumped I-V curve is calculated using any admittance taken from inside the the innermost contour, it is visually indistinguishable from the experimentally measured pumped I-V curve. 


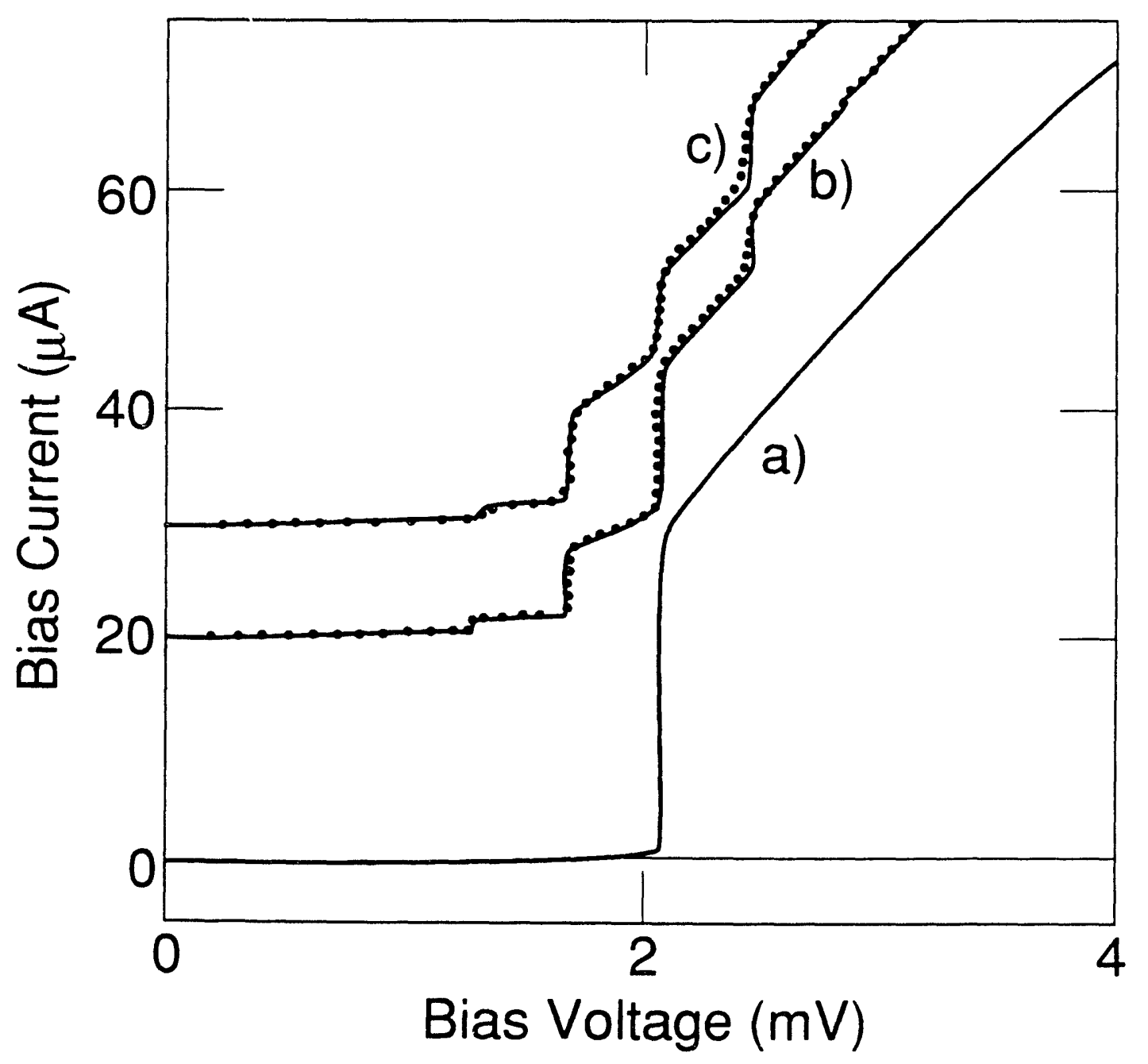

Fig. 4.5 a) dc I-V curve of an SIS junction measured at Temperature 1.3 K. b) experimental and calculated pumped I-V curves. The solid line is the calculated curve. The experimental points are represented by dots. The pump frequency is $96.35 \mathrm{GHz}$. The embedding admittance used in the calculation is $\mathrm{Y}_{\mathrm{emb}}=0.14+$ $0.08 \mathrm{i} \Omega^{-1}$. c) same as b), except here the pump frequency is 93.65 $\mathrm{GHz}$, and the embedding admittance used is $\mathrm{Y}_{\mathrm{emb}}=0.04+0.18 \mathrm{i}$ $\Omega^{-1}$. 


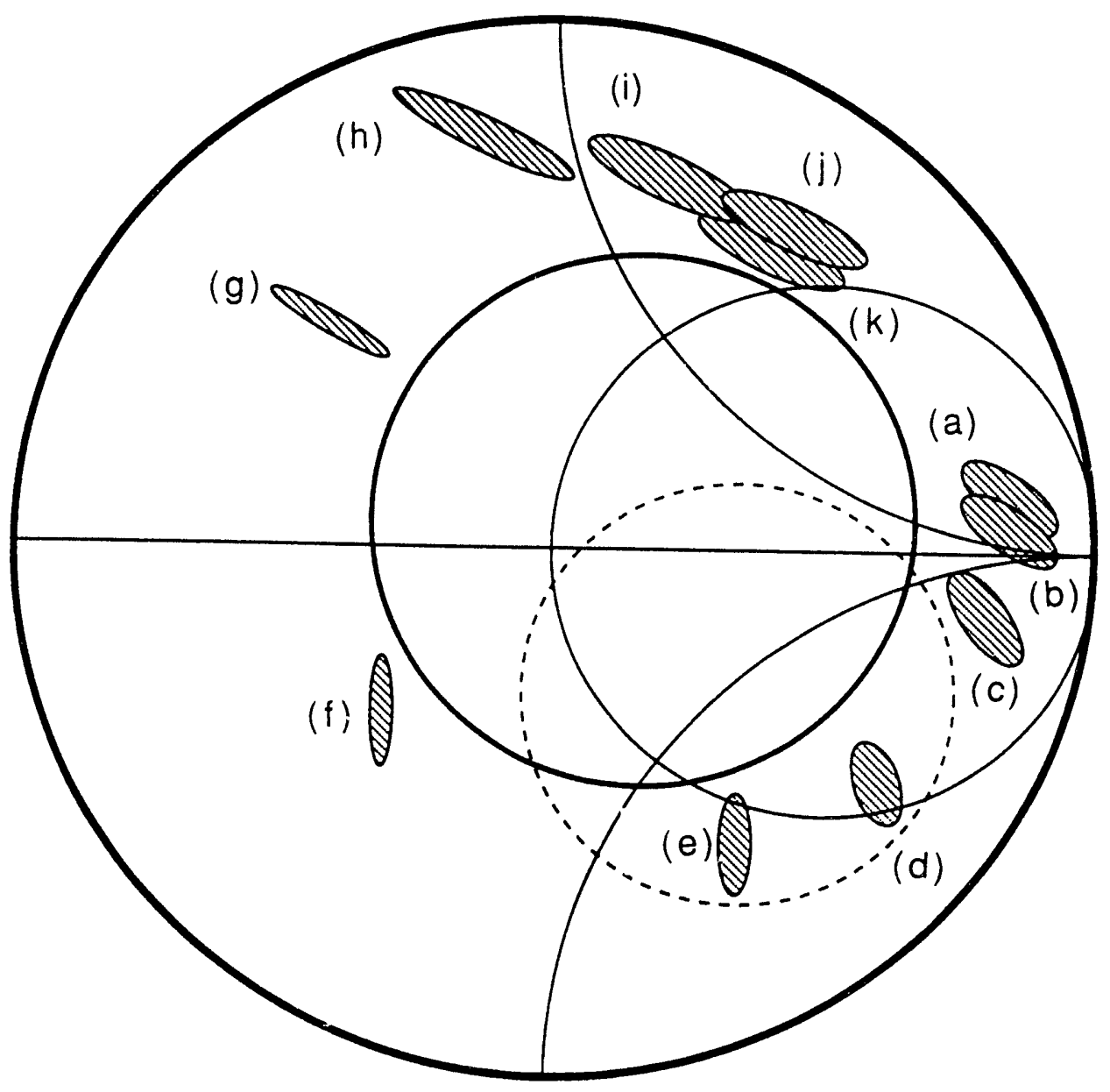

Fig 4.6 Comparison between admittances measured using a scaled model and those deduced from pumped I-V curves. This analysis was done for our waveguide mixer block at $93 \mathrm{GHz}$. The shaded regions (a) - (1) represent the ranges of embedding admittance deduced from a series of pumped I-V curves measured, each with the backshort stepped $0.0165 \mathrm{~mm}$ farther away from the junction. The dashed circle is the range of available embedding admittances measured from the scaled model measured at a scaled frequency corresponding to $93 \mathrm{GHz}$. The solid circle is the same range, except that the susceptance due to the estimated geometrical capacitance of the junction of $160 \mathrm{fF}$ has been added in parallel. 


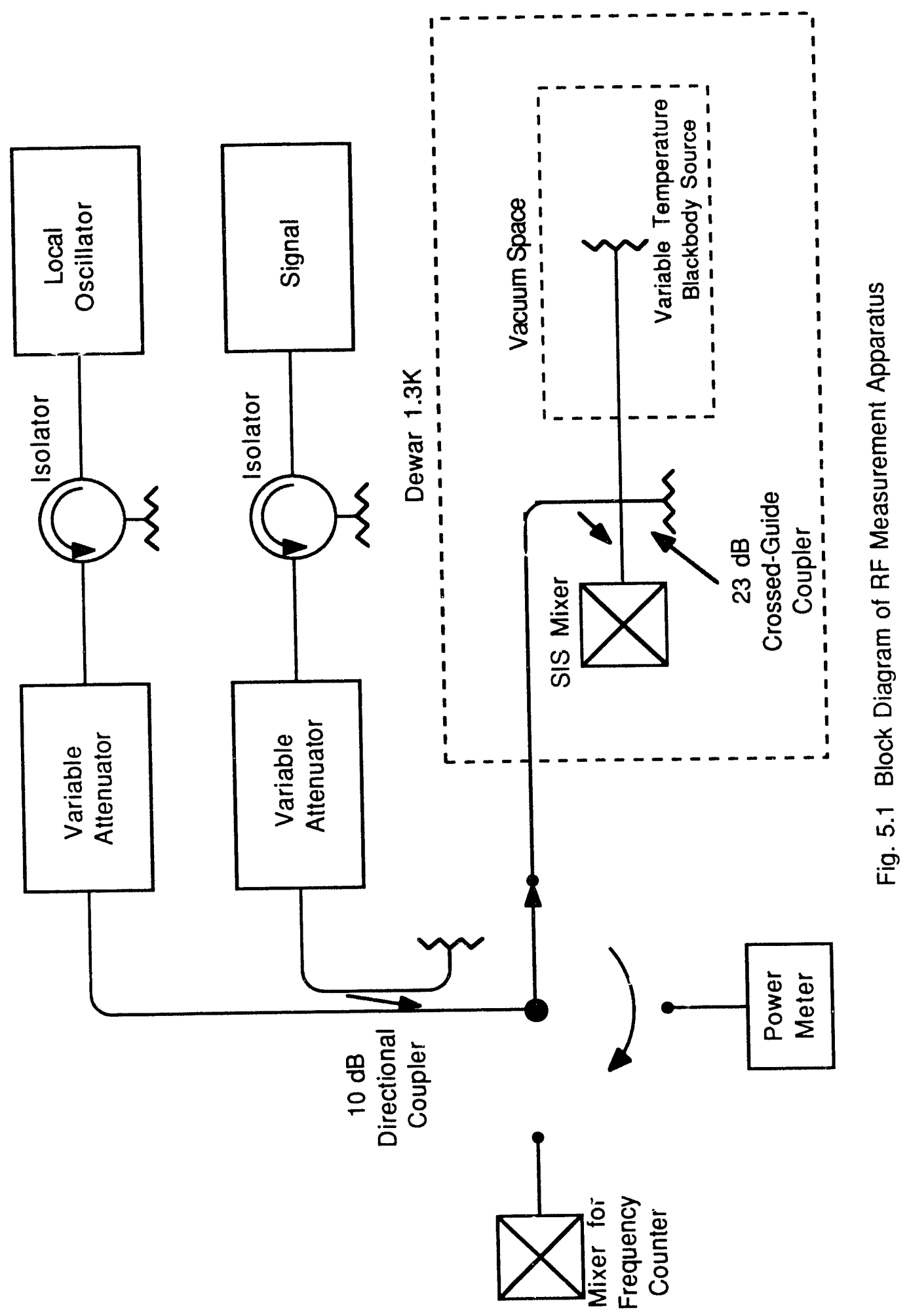




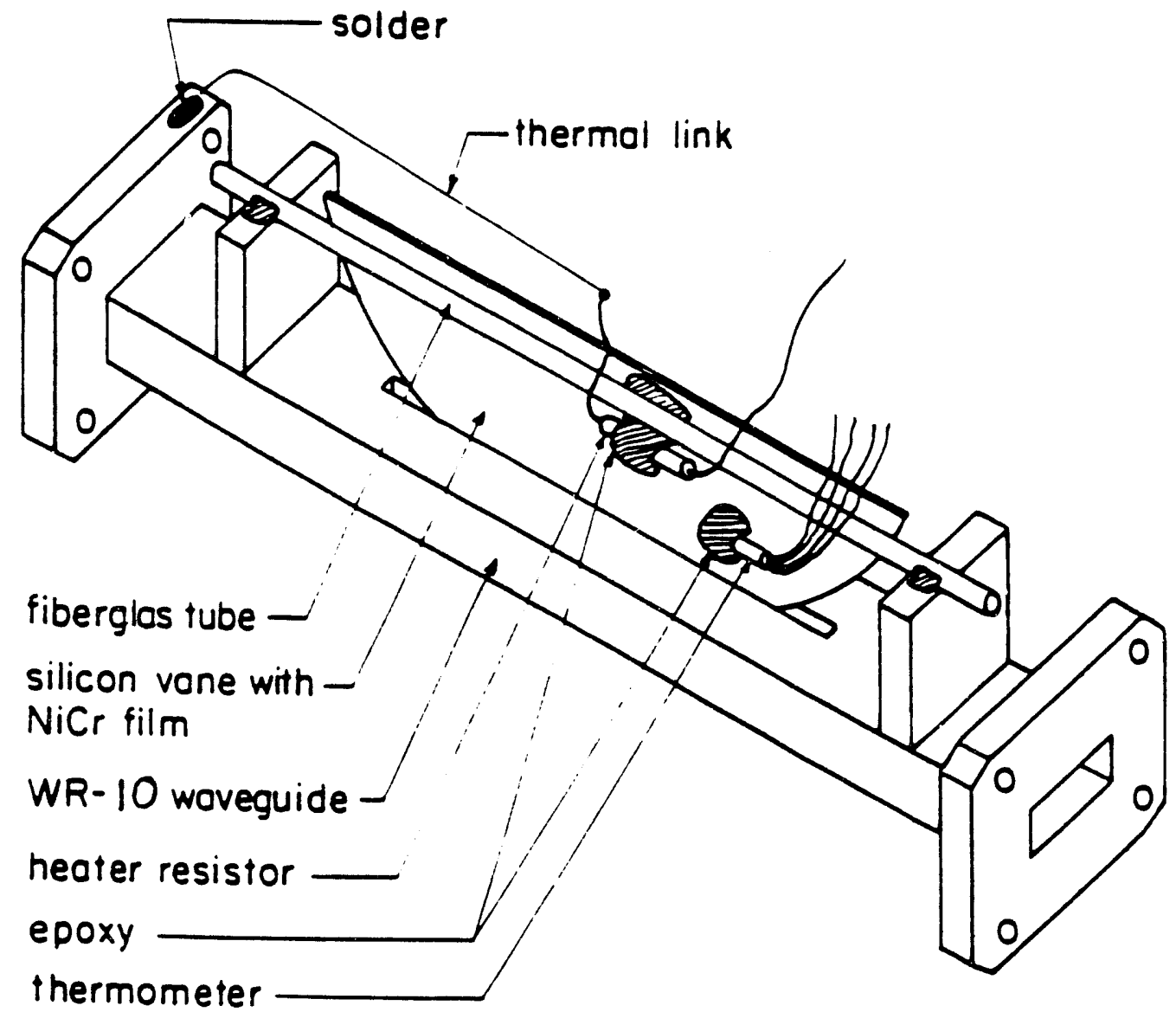

Fig. 5.2 Variable temperature RF waveguide load used to produce a calibrated blackbody signal for accurate measurements of mixer performance. 


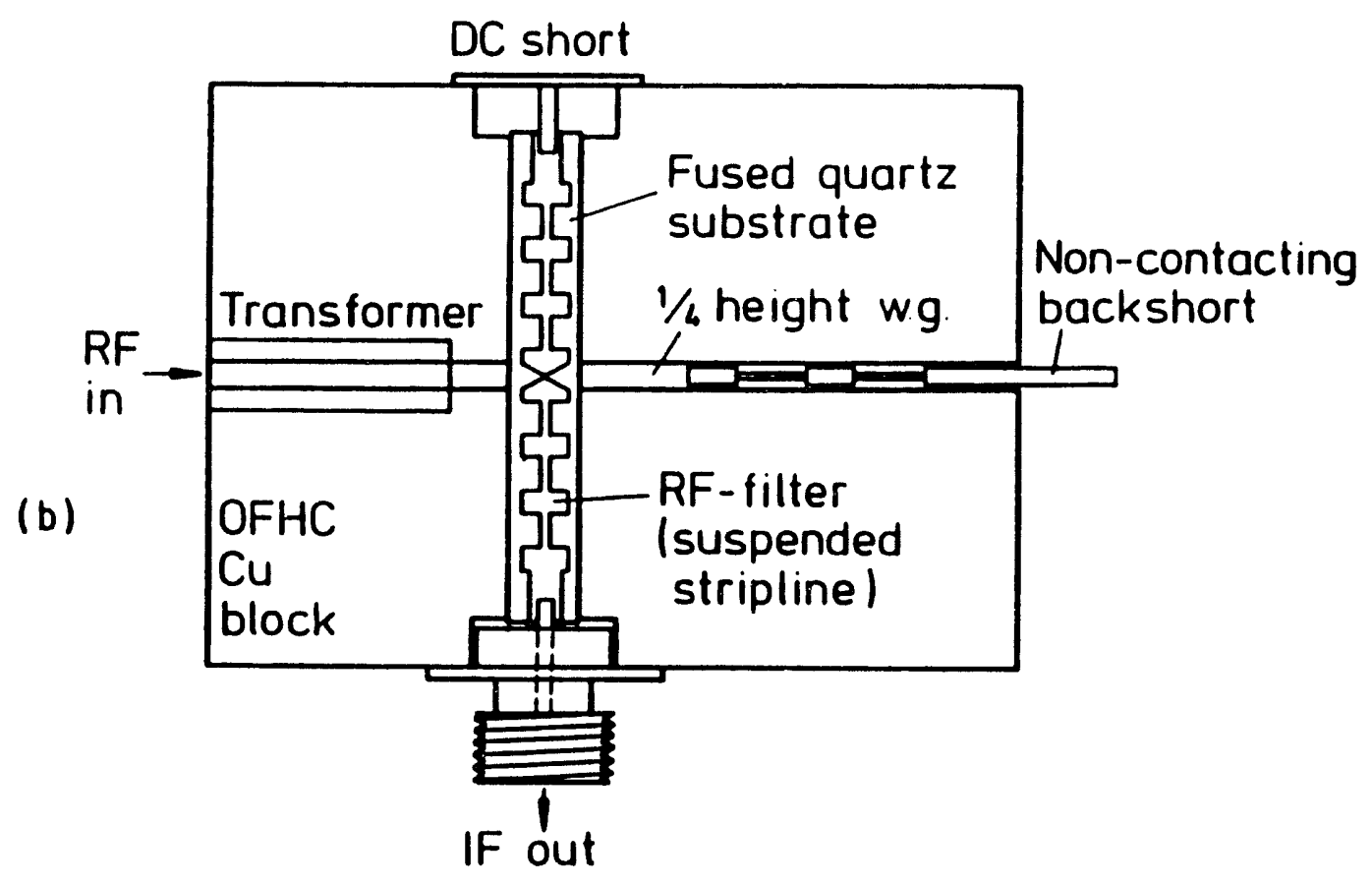

Fig. 5.3 Diagram of the mixer block used for $90 \mathrm{GHz}$ waveguide mixer experiments. 


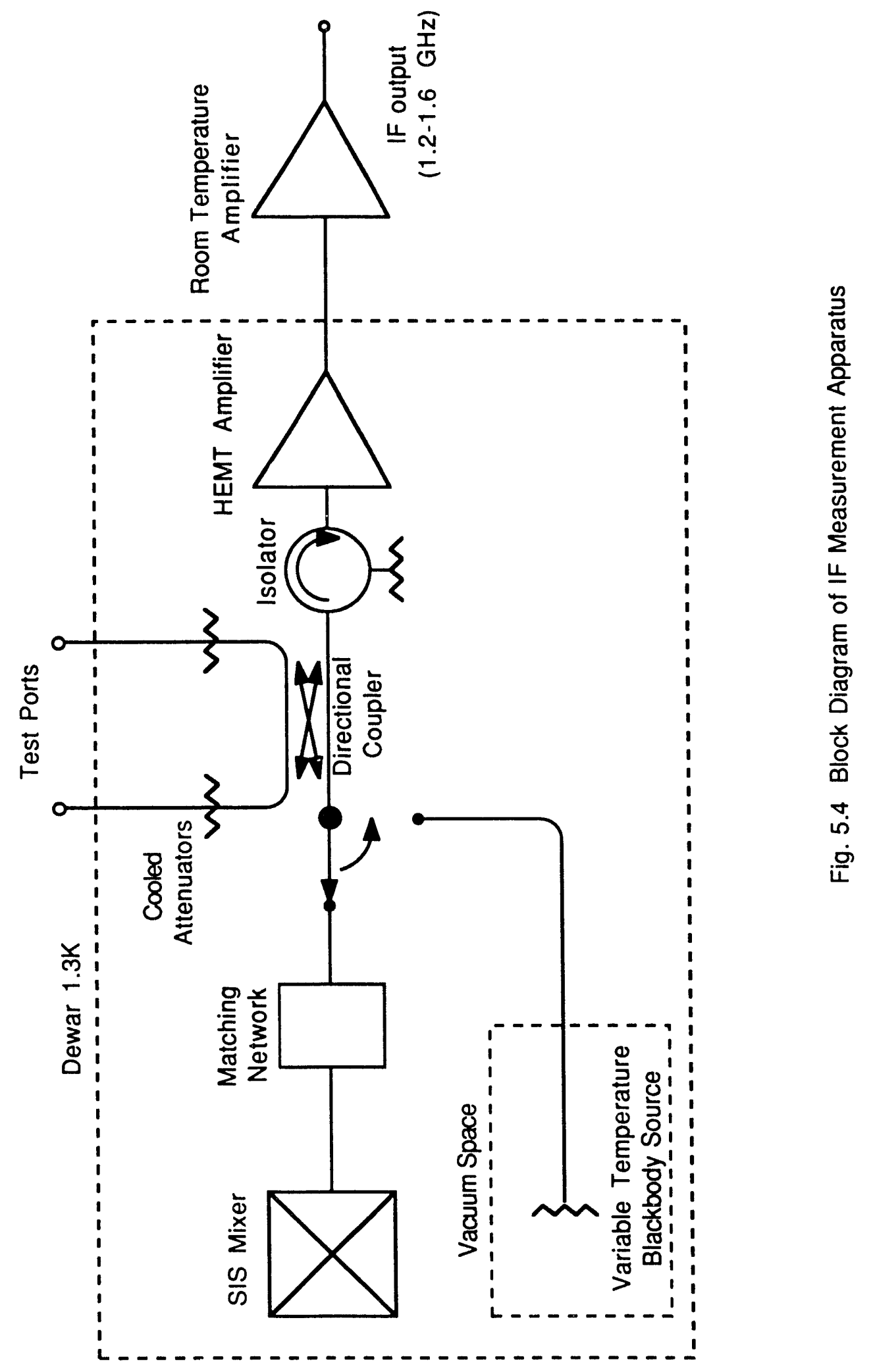




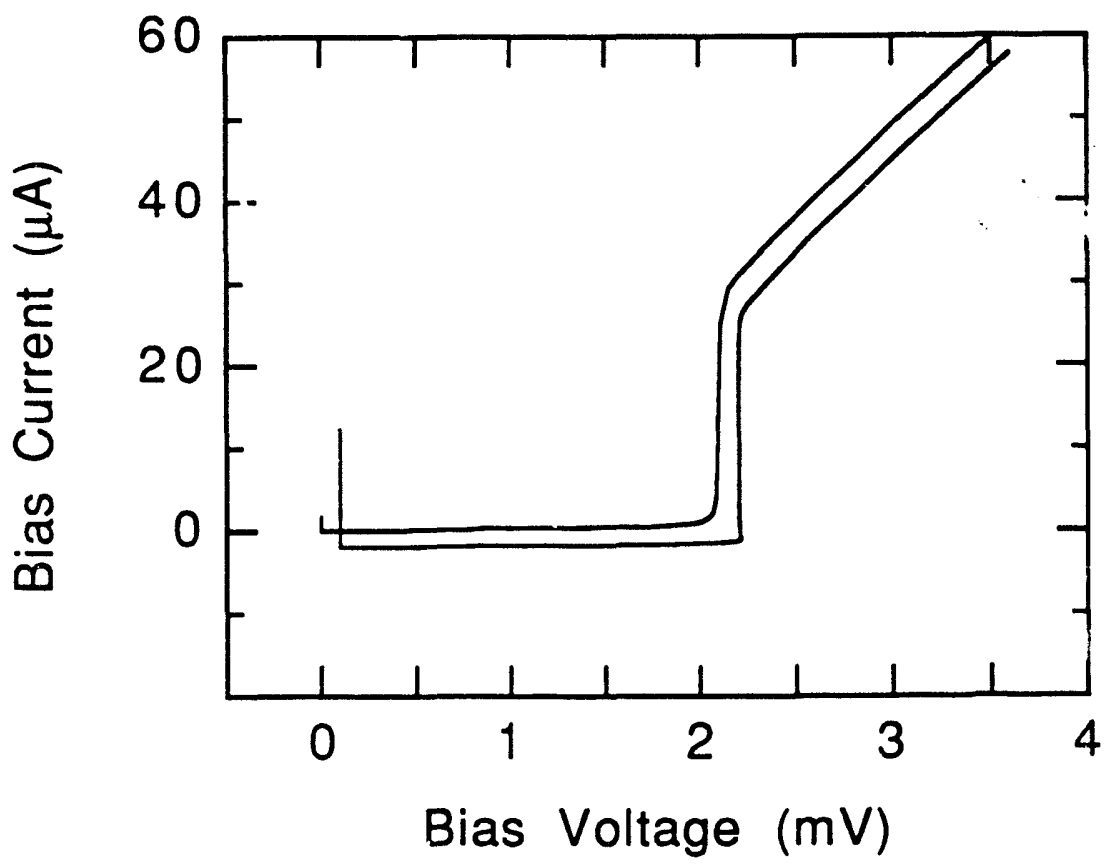

Fig. 5.5 Two dc I-V curves measured from the tantalum junction used. Both curves were measured at $1.3 \mathrm{~K}$ with no applied magnetic field. The two curves differ because different amounts of magnetic flux were trapped in the junction. 


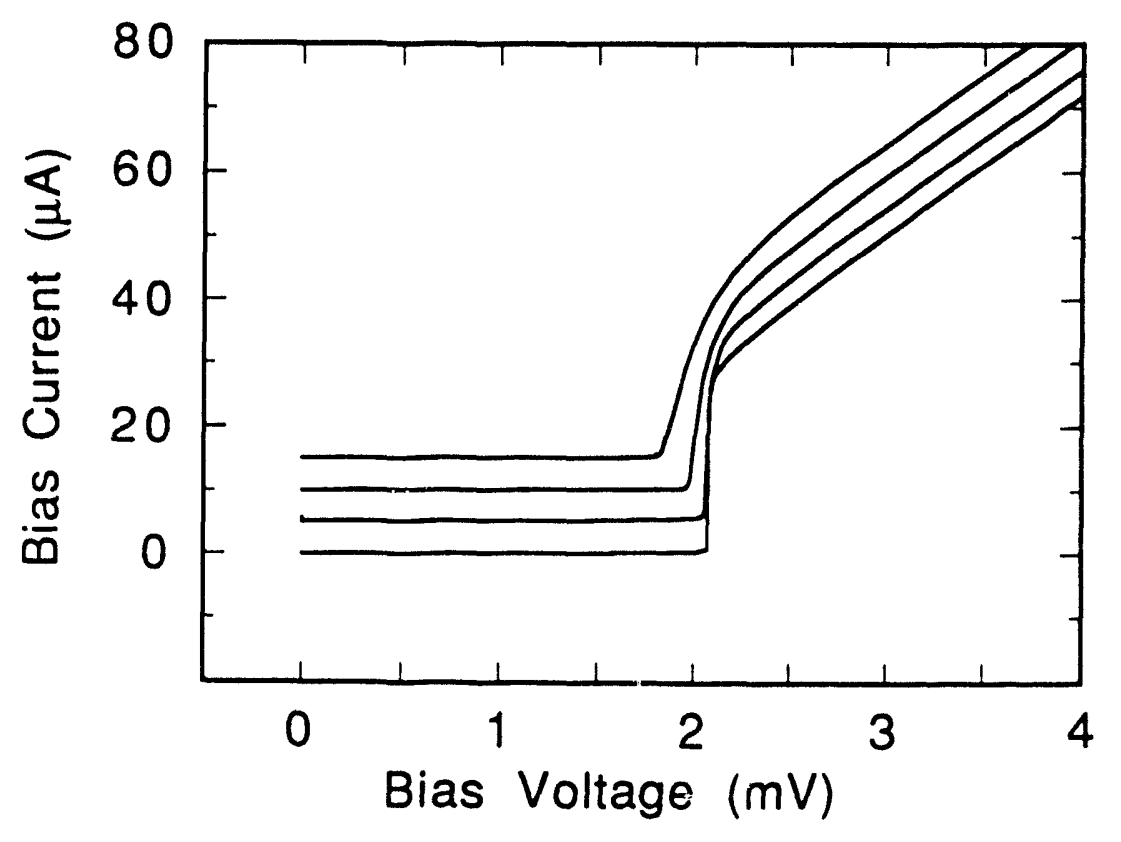

Fig. 5.6 A series of dc I-V curves measured with different values of the applied magnetic field. As the field is increased from zero, the density of states near the gap begins to smear, leading to a more rounded current rise. 


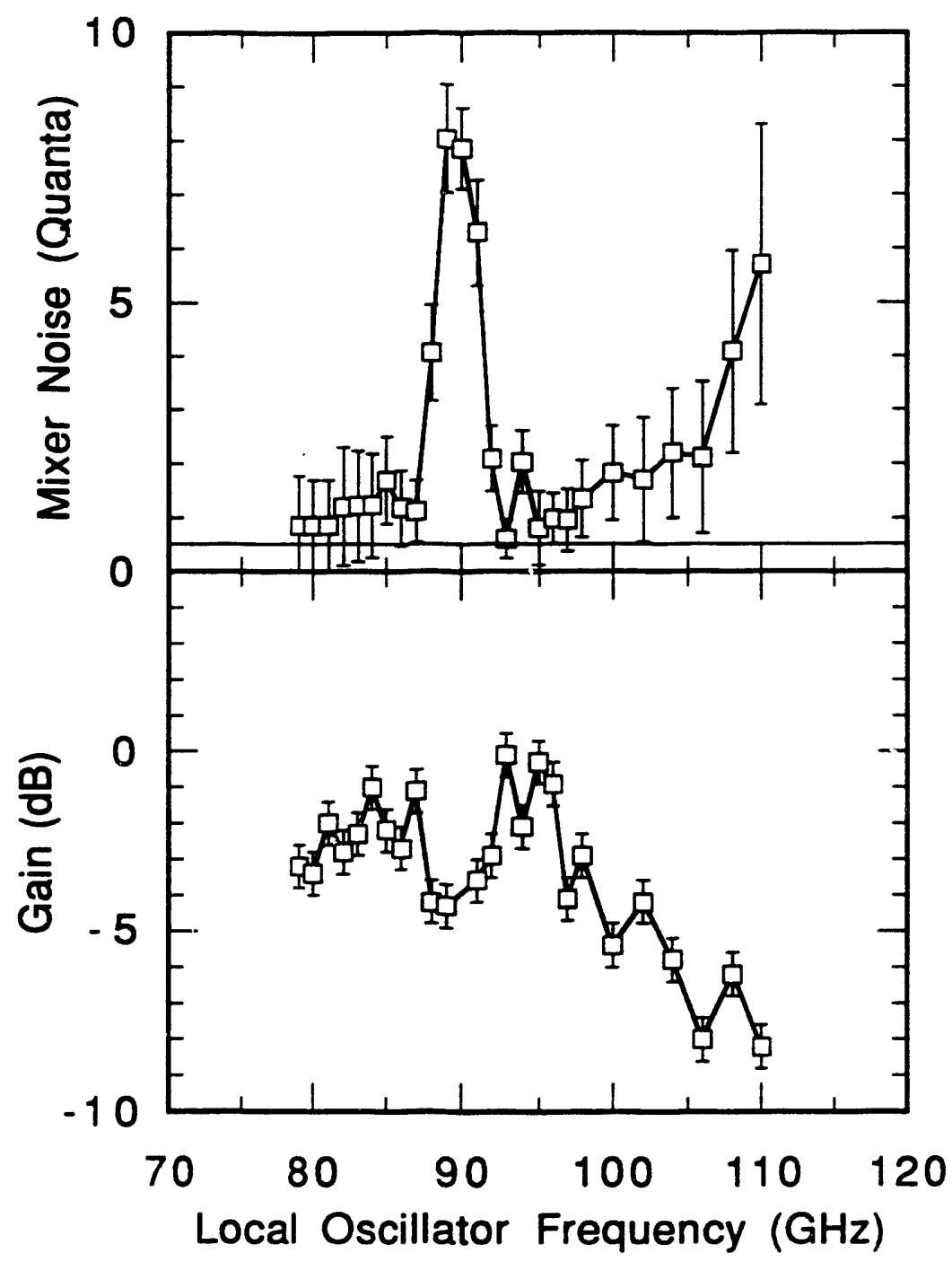

Fig. 5.7 Added mixer noise and available gain plotted as a function of local oscillator frequency. At each frequency, the local oscillator power, dc bias vcltage and backshort position were optimized for maximum coupled gain. The peak in mixer noise near $90 \mathrm{GHz}$ corresponds to a resonance in our mixer block which makes it impossible to provide favorable embedding admittances. The horizontal line at $S=1 / 2$ is the quantum limit imposed by the uncertainty principle. The $S=1 / 2$ vacuum fluctuations already present on the signal are not included in the mixer noise. 


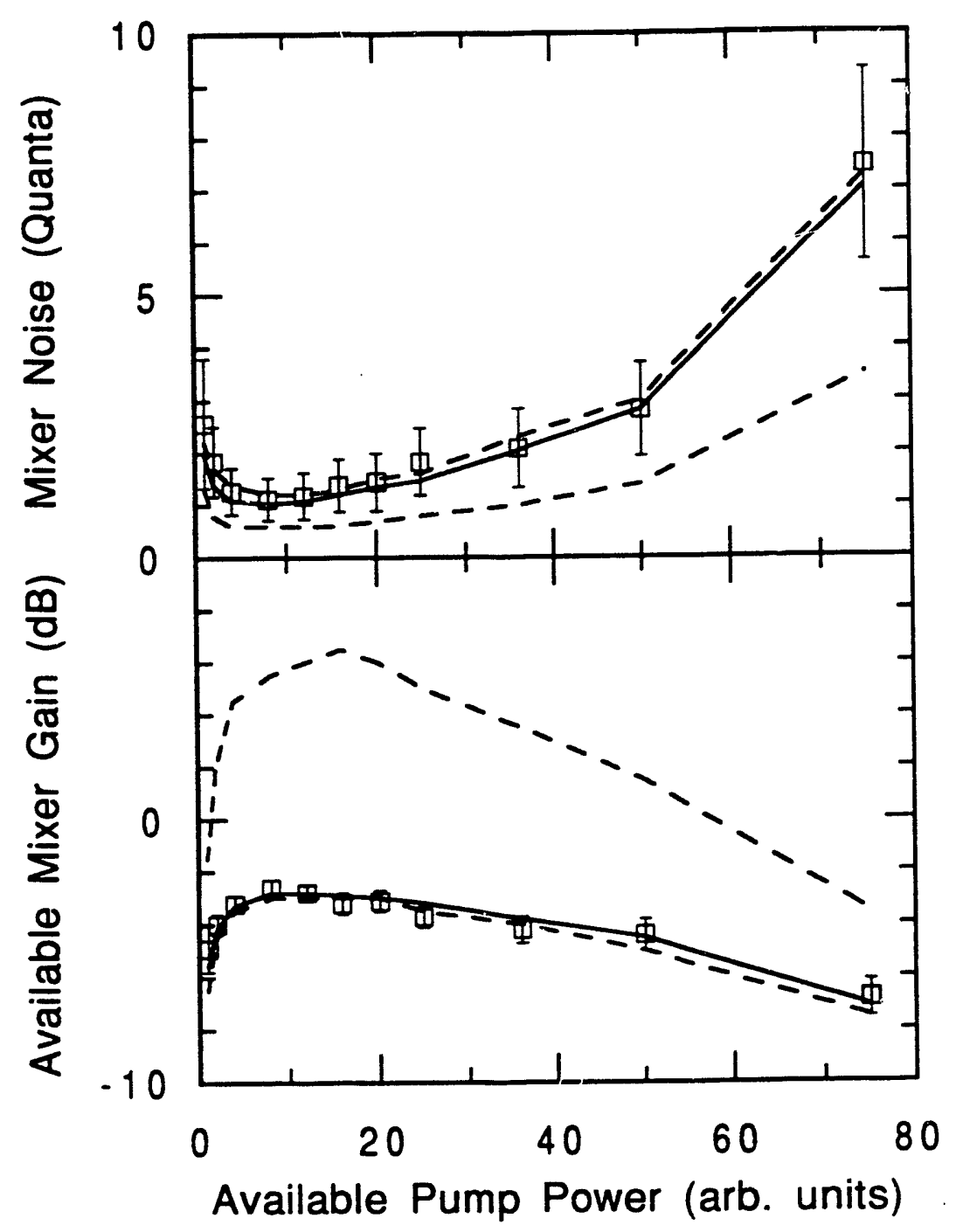

Fig 5.8 Added mixer noise and available gain as a function of local oscillator power with $\mathrm{fLO}_{\mathrm{LO}}=95.0 \mathrm{GHz}, \mathrm{VDC}_{\mathrm{DC}}=1.956 \mathrm{mV}$. The dashed lines are the limits of the performance that are consistent with I-V curve shape. The solid line is the best fit to measured performance, with $Y_{U S B}=0.14+0.08 i \Omega^{-1}$ and $Y_{L S B} 0.04+0.18 i$ $\Omega^{-1}$. All measurements were performed with no applied magnetic field. 


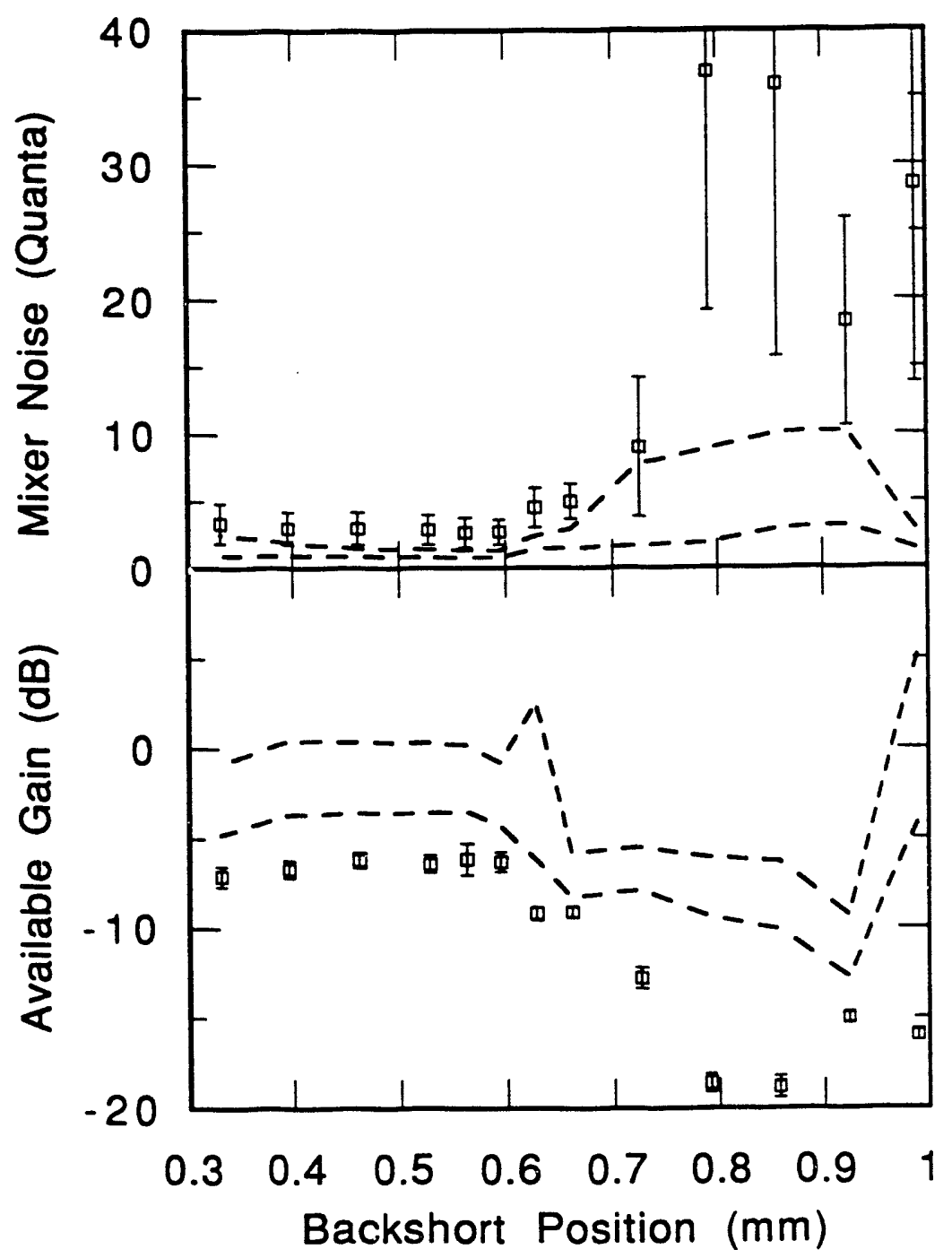

Fig. 5.9 Added mixer noise and available gain as a function of backshort posiiton. The dashed lines are the limits of performance that are consistent with I-V curve shape. The fit is relatively poor for backshort positions more than $0.59 \mathrm{~mm}$ from the junction. 


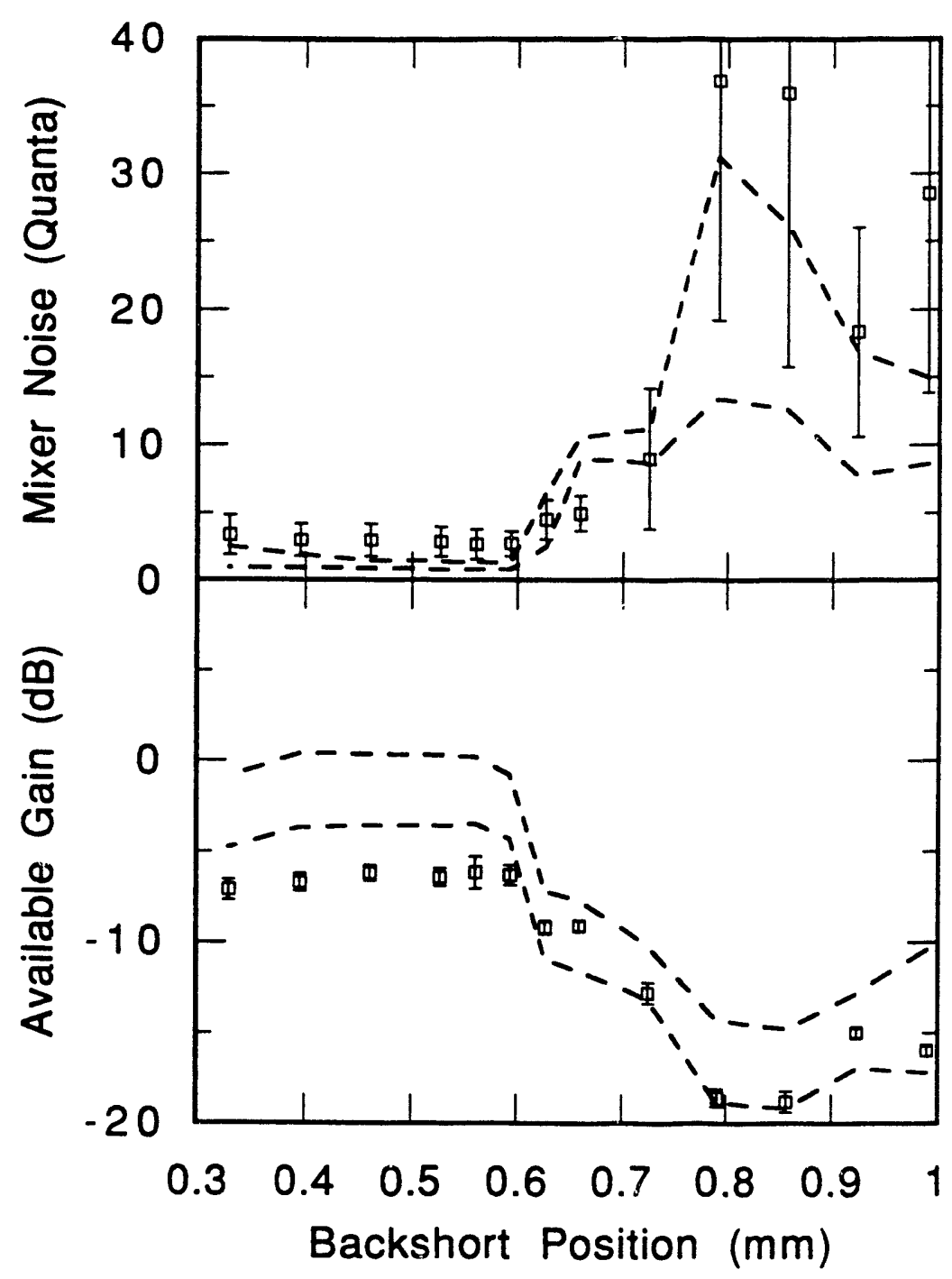

Fig. 5.10 Same as Fig. 5.9 except that now the calculated limits of performance are calculated using only admittances that are consistent with measured values of pump power for backshort position more than $0.59 \mathrm{~mm}$ from the junction. Note that the quality of the fit is dramatically improved in this region. 


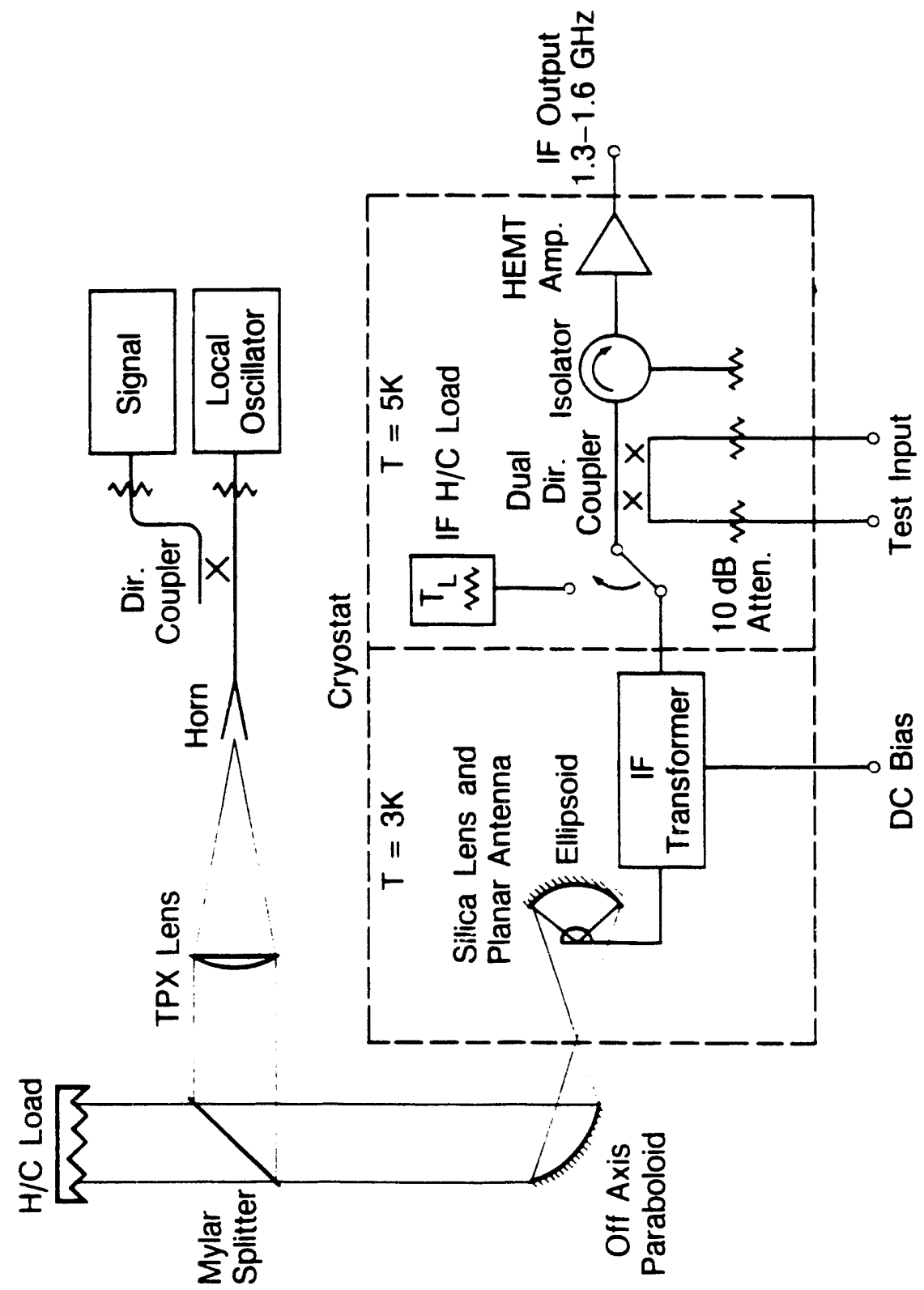

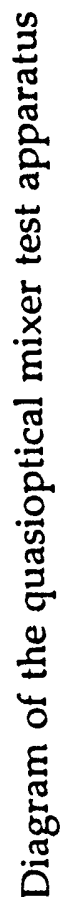

70
0
00 
(a)

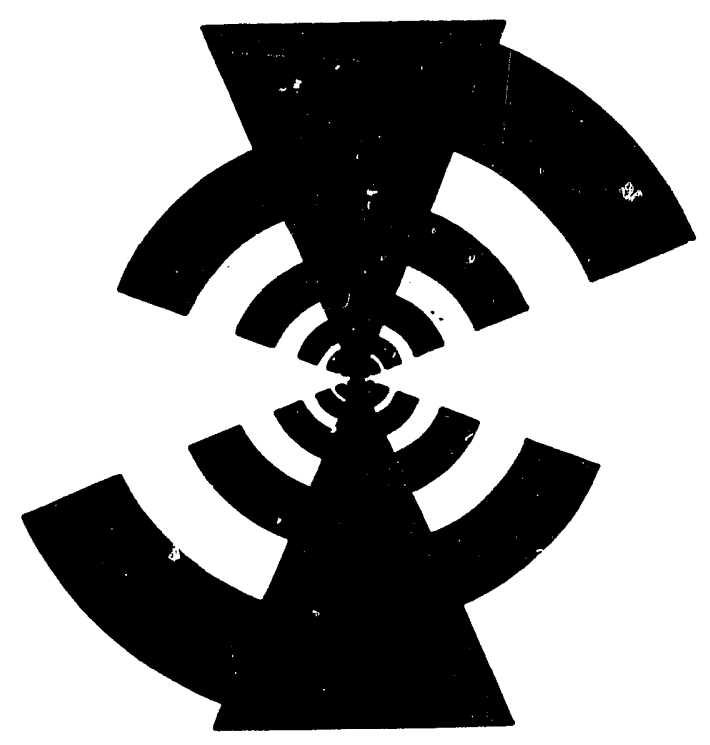

(b)

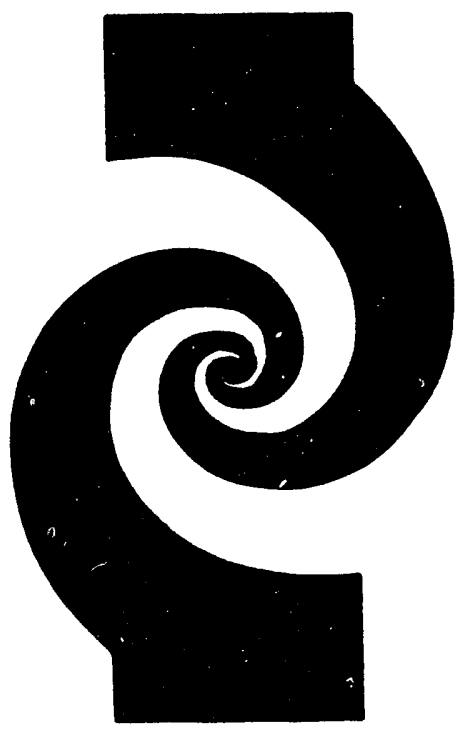

Fig. 6.2 a) Circular-toothed planar log-periodic antenna. b) twoarm log-spiral antenna. 


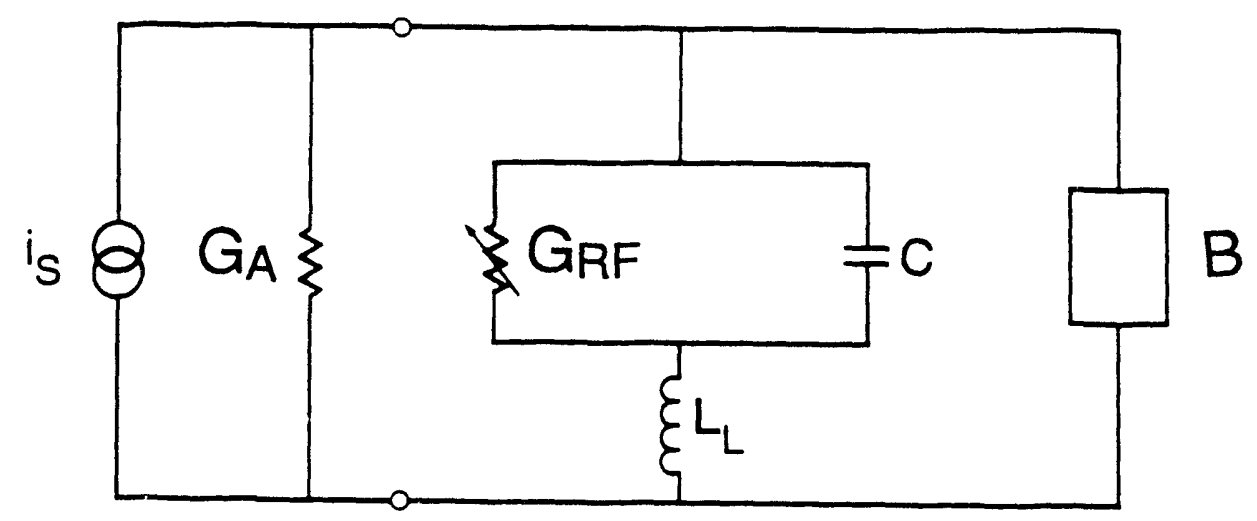

Fig. 6.3 Equivalent circuit used to analyze the performance of quaisoptical mixers. The signal at the terminals of the planar antenna is represented by the current generator $i_{S}$ and the conductance $G_{A}$. The mixing element is represented by the non-linear conductance $G_{R F}$ and the geometrical capacitance $C$, the leads of the junction by $L_{L}$, and the tuning element by the susceptance $B$. 

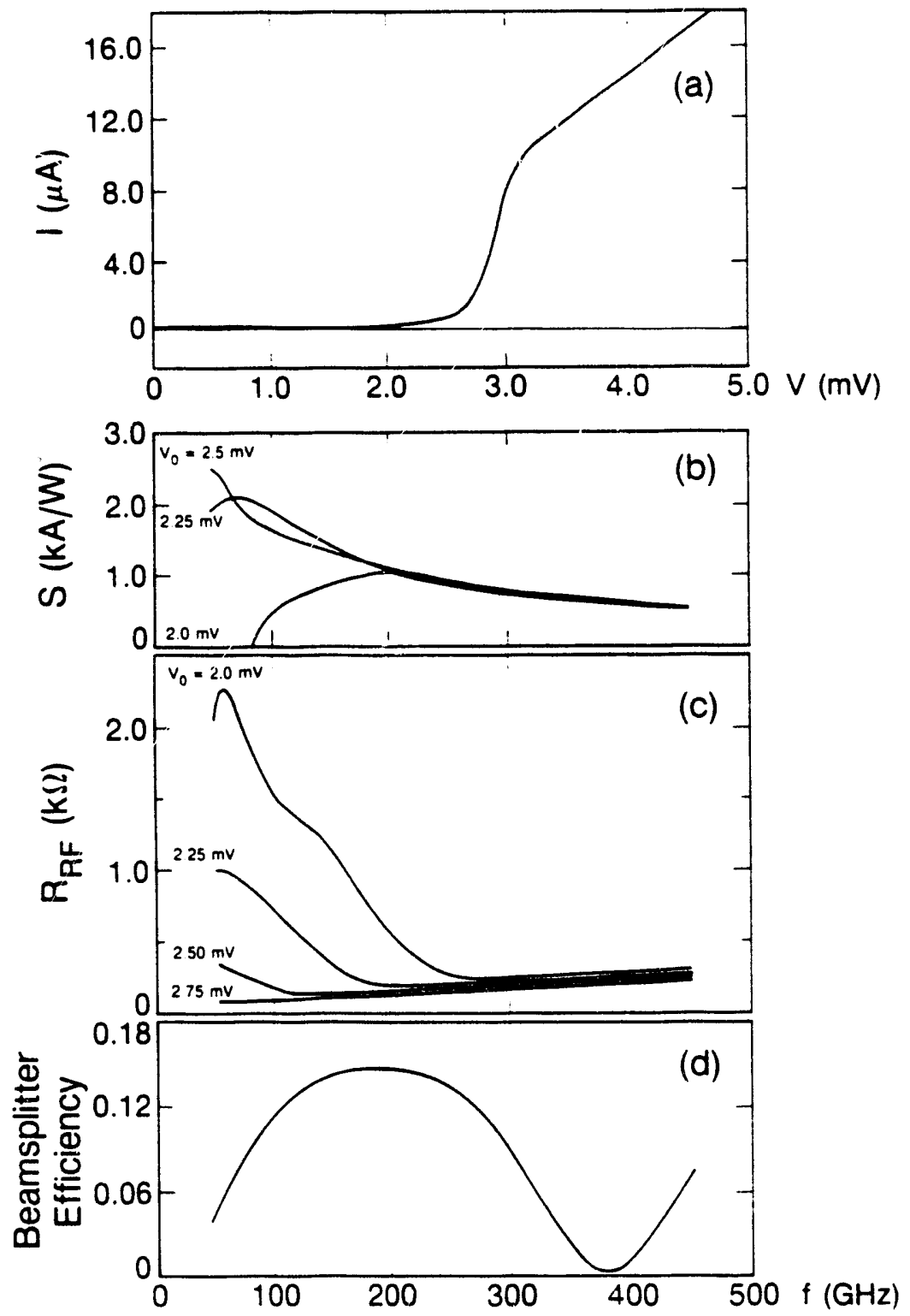

Fig. 6.4 a) dc I-V curve of a typical SIS junction. b) Computed frequency dependence of the current responsivity $S\left(\omega, V_{0}\right)$ at several values of bias voltage $V_{0}$. c) Computed frequency dependence of the $R F$ resistance $R_{R F}$ at several dc bais voltages. d) Computed beamsplitter efficiency for a $250 \mu \mathrm{m}$ thick Mylar sheet at a $45^{\circ}$ angle of incidence. 


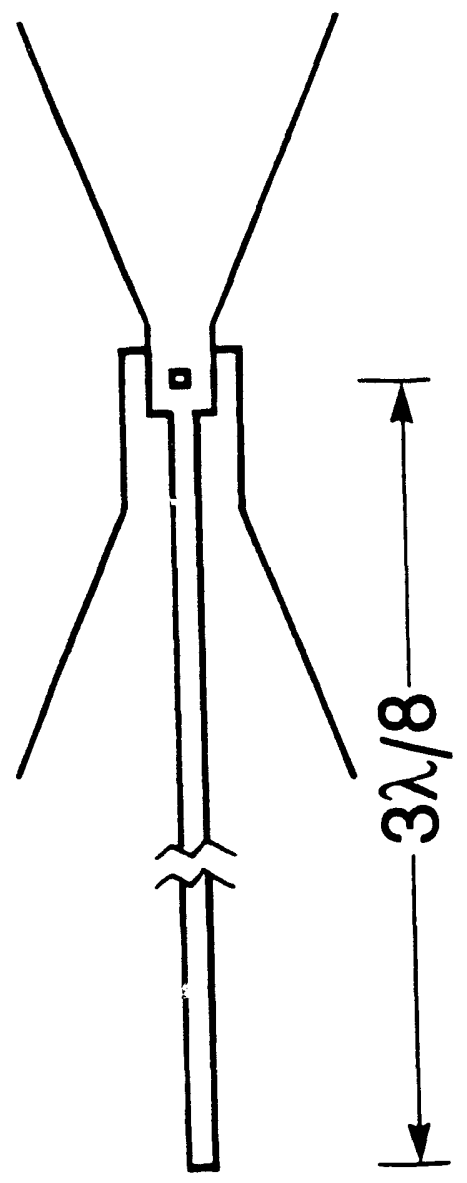

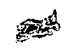

Fig. 6.5 Diagram of and SIS junction with a $3 \lambda / 8$ open-ended microstrip stub used as an inductive tuning element. The junction is shown at the terminals of a log-periodic antenna. 


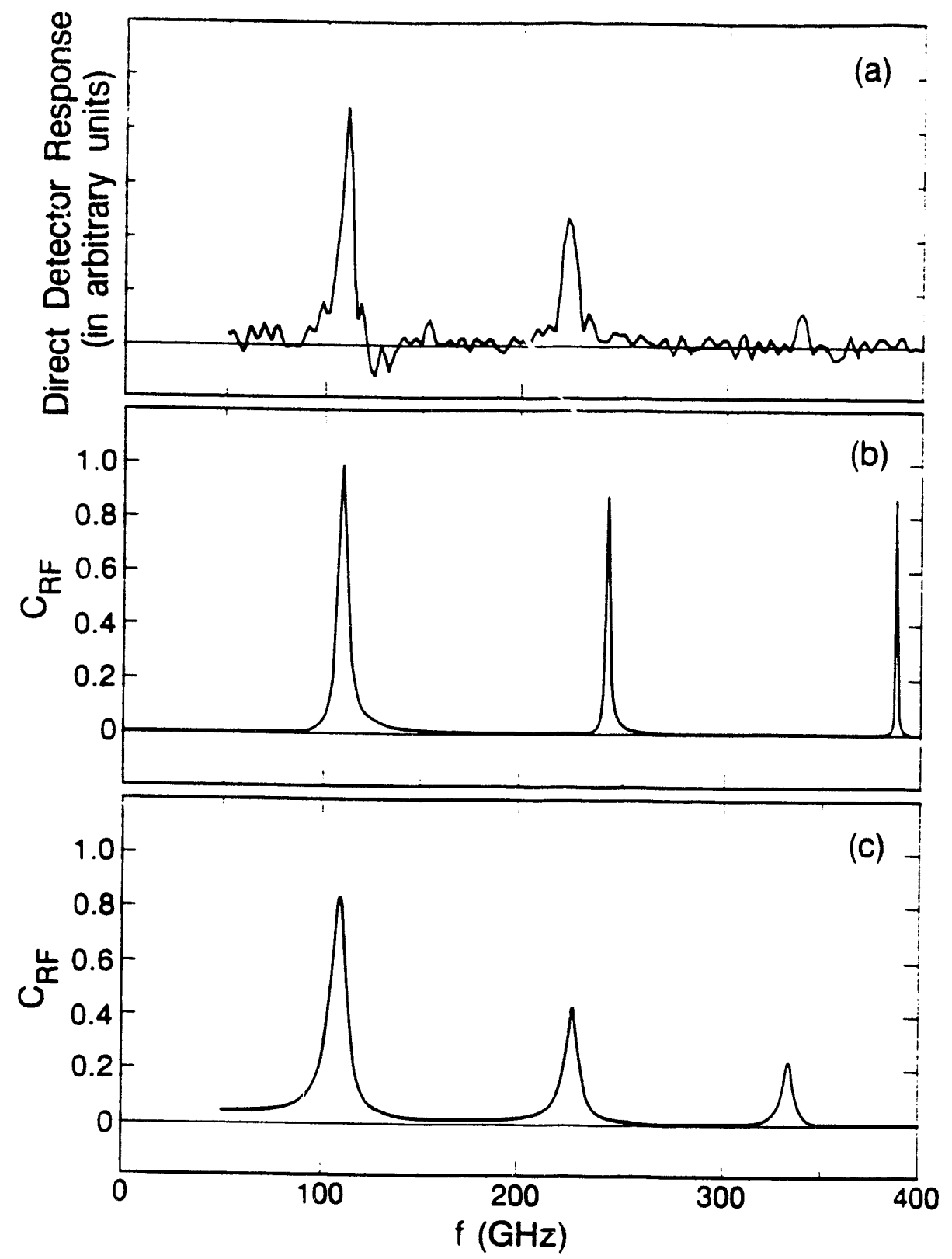

Fig. 6.6 a) Measured frequency response of single junction with a $3 \lambda / 8$ stub. b) Computed coupling coefficient $C_{R F}$ for a single junction with a lossless open-circuited microstrip stub. c) Computed $\mathrm{C}_{\mathrm{RF}}$ for a single junction with a lossy, dispersive stub with parameters indicated in the text. 


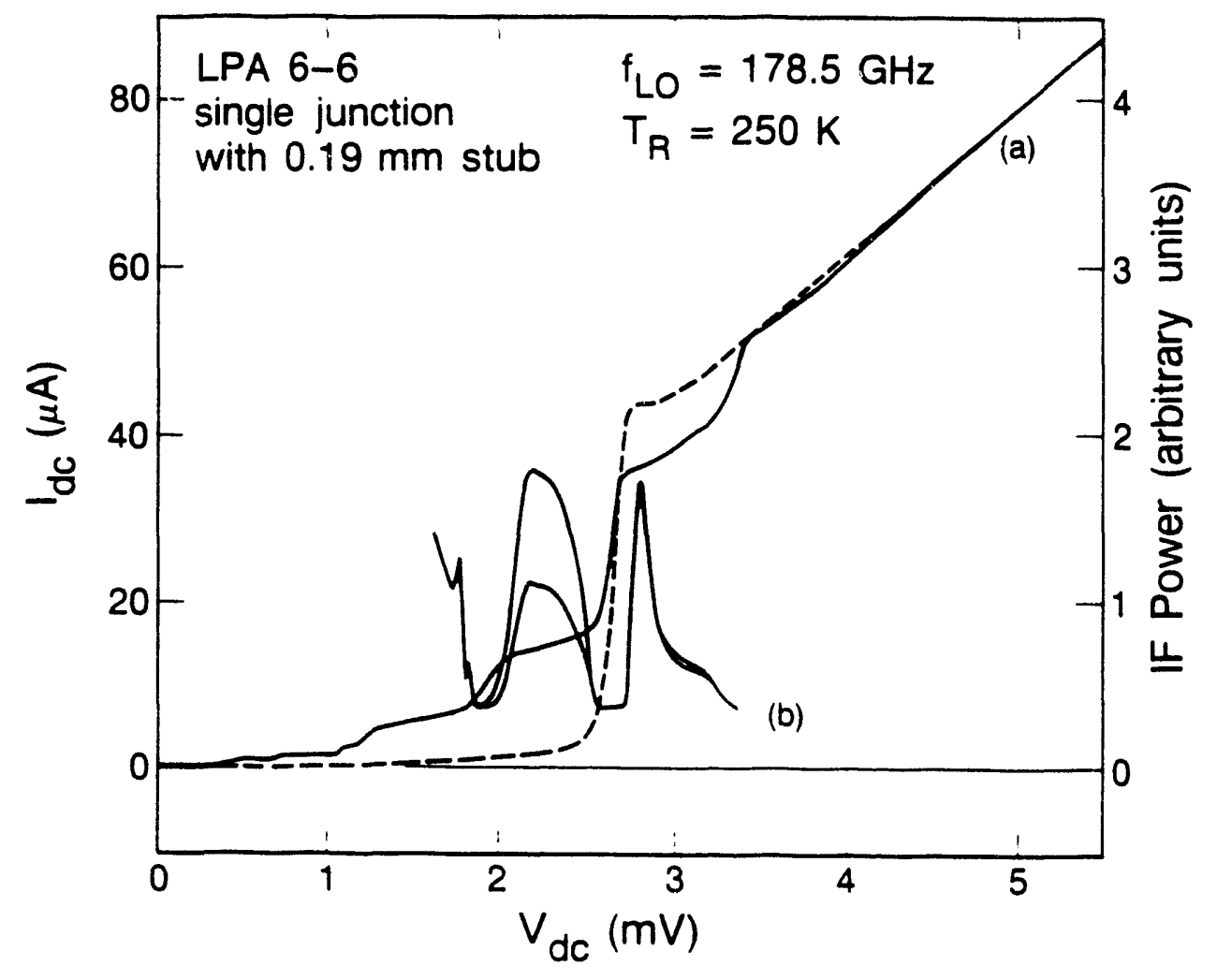

Fig. 6.7 a) I-V curves of a pumped (solid line) and unpumped junction. b) IF output power as a function of dc bias. The upper curve is for the hot $(300 \mathrm{~K})$ and the lower curve is for the cold (77 K) RF load. 


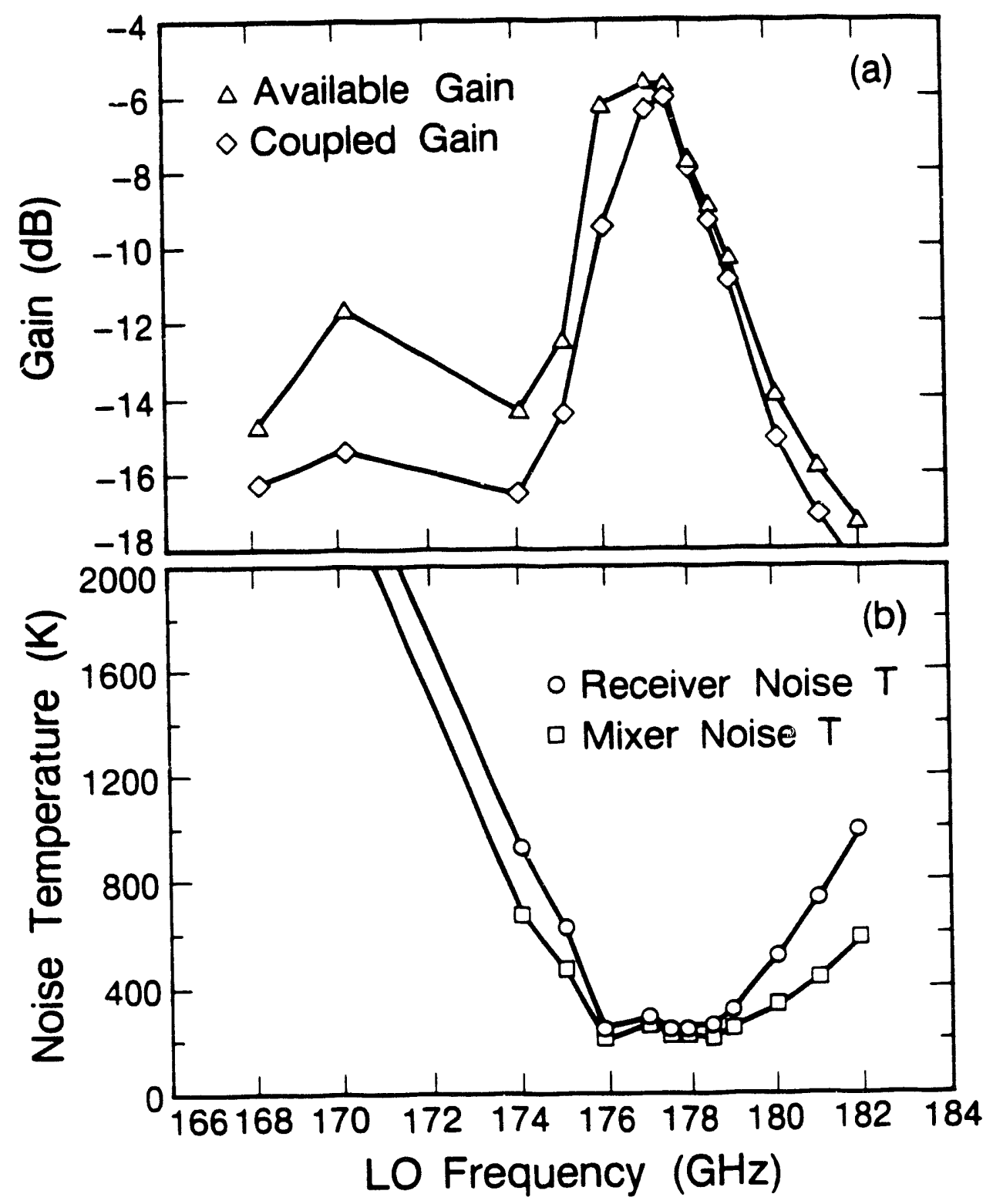

Fig. 6.8

a) Coupled and available mixer gain as function of LO frequency. b) Mixer and receiver noise temperature functions of LO frequency. 


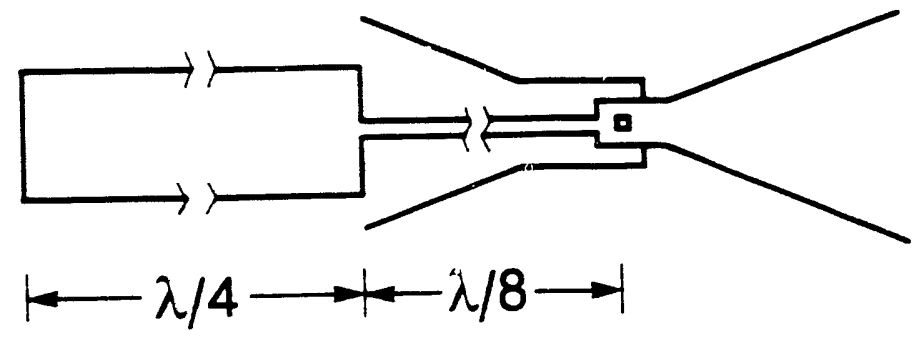

(a)

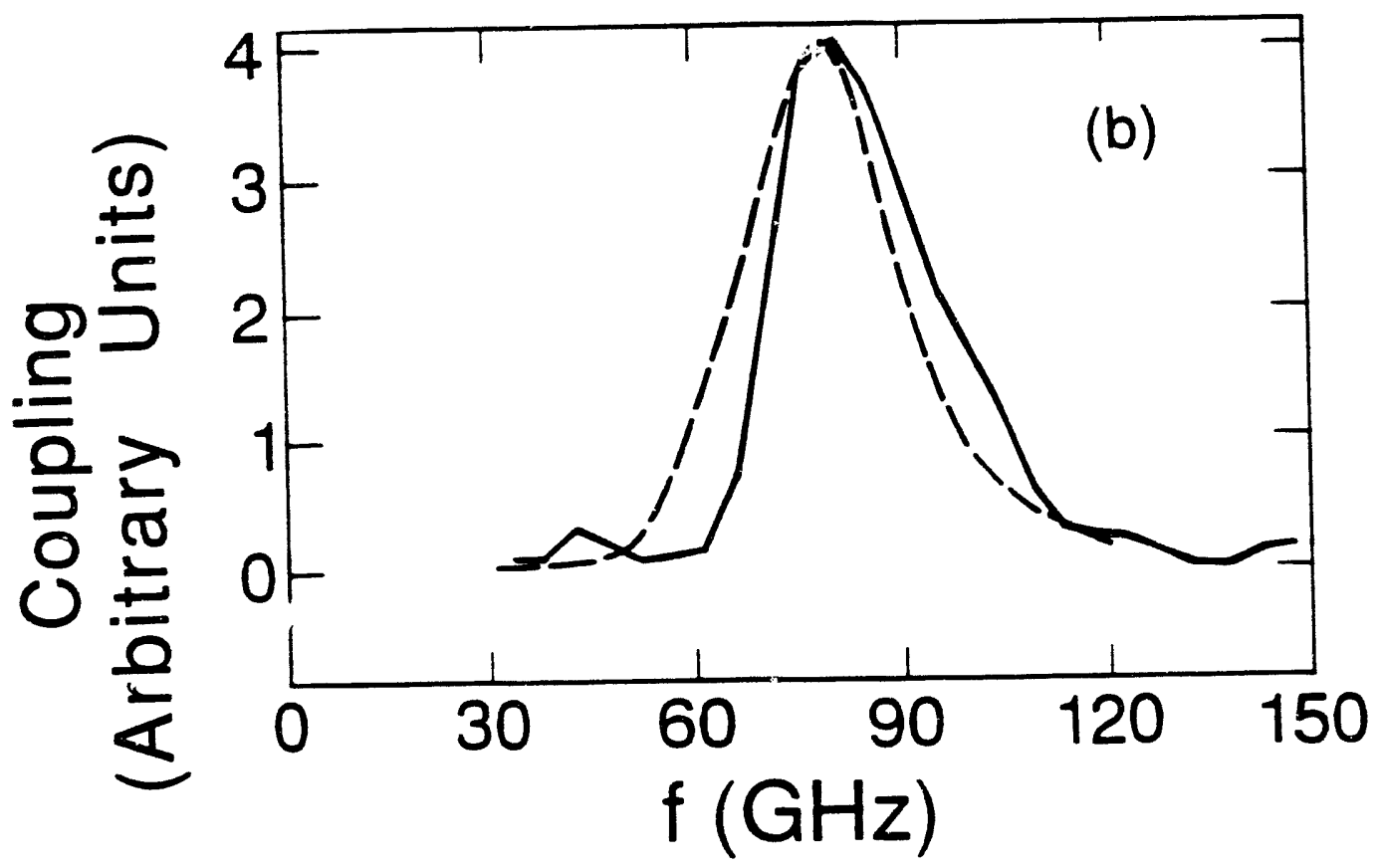

Fig. 6.9 a) Diagram of an SIS junction with a $\lambda / 8$ stub used as an inductive tuning element. The wide section of microstrip provides a broadband RF short for the $\lambda / 8$ stub. b) Measured (Solid line) and computed (dashed line) frequency response of the structure shown in a). 
(a)
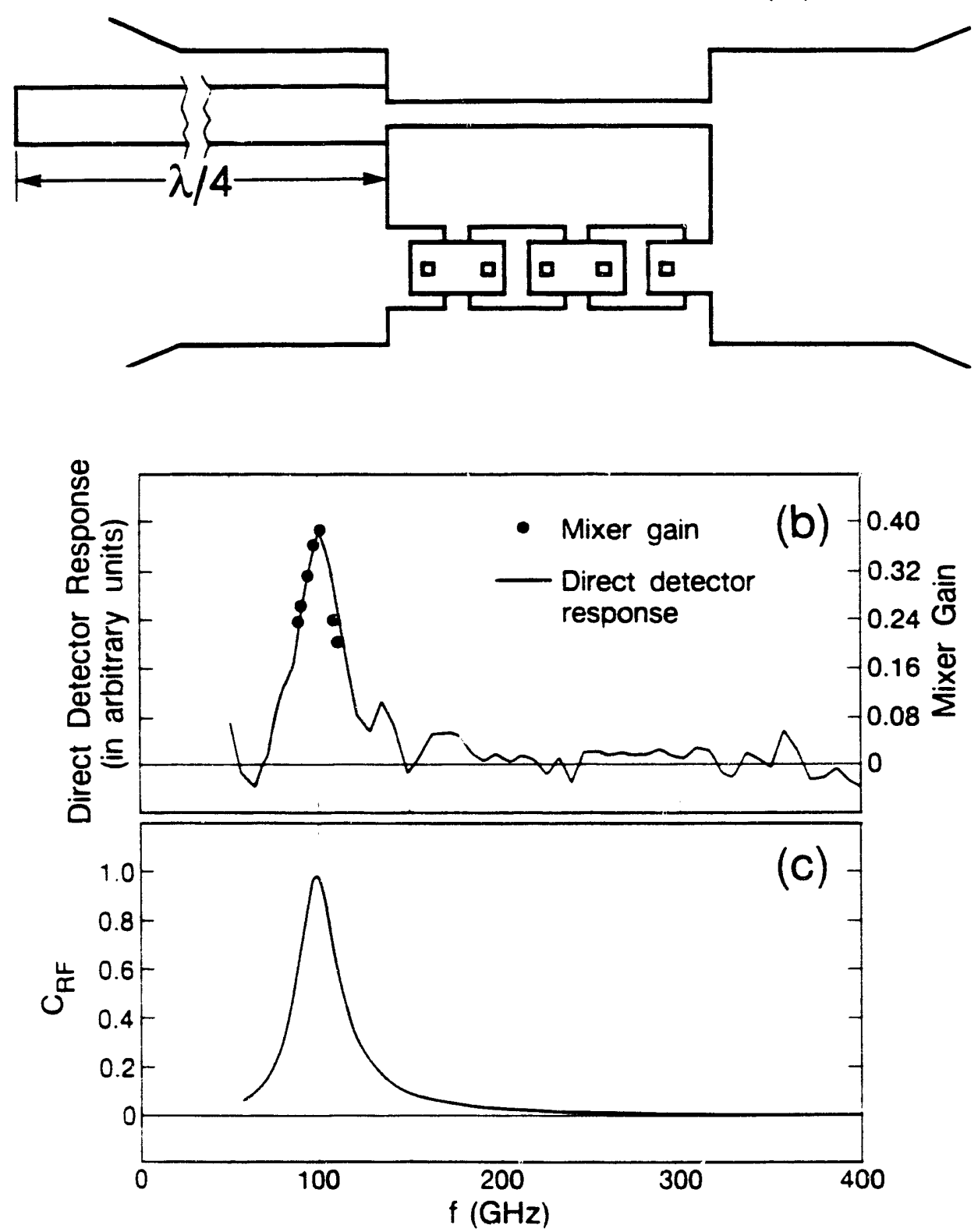

Fig. $6.10 \quad$ a) Diagram of an 5-junction array of SIS junctions with an inductive wire as a tuning element. The wire is RF-shorted by a $\lambda / 4$ section of microstrip transmission line. b) Measured frequency response of the structure shown in a).

c) Computed RF coupling coefficient of $\mathrm{t}^{\mathrm{t}}$.e structure shown in a). 
(a)
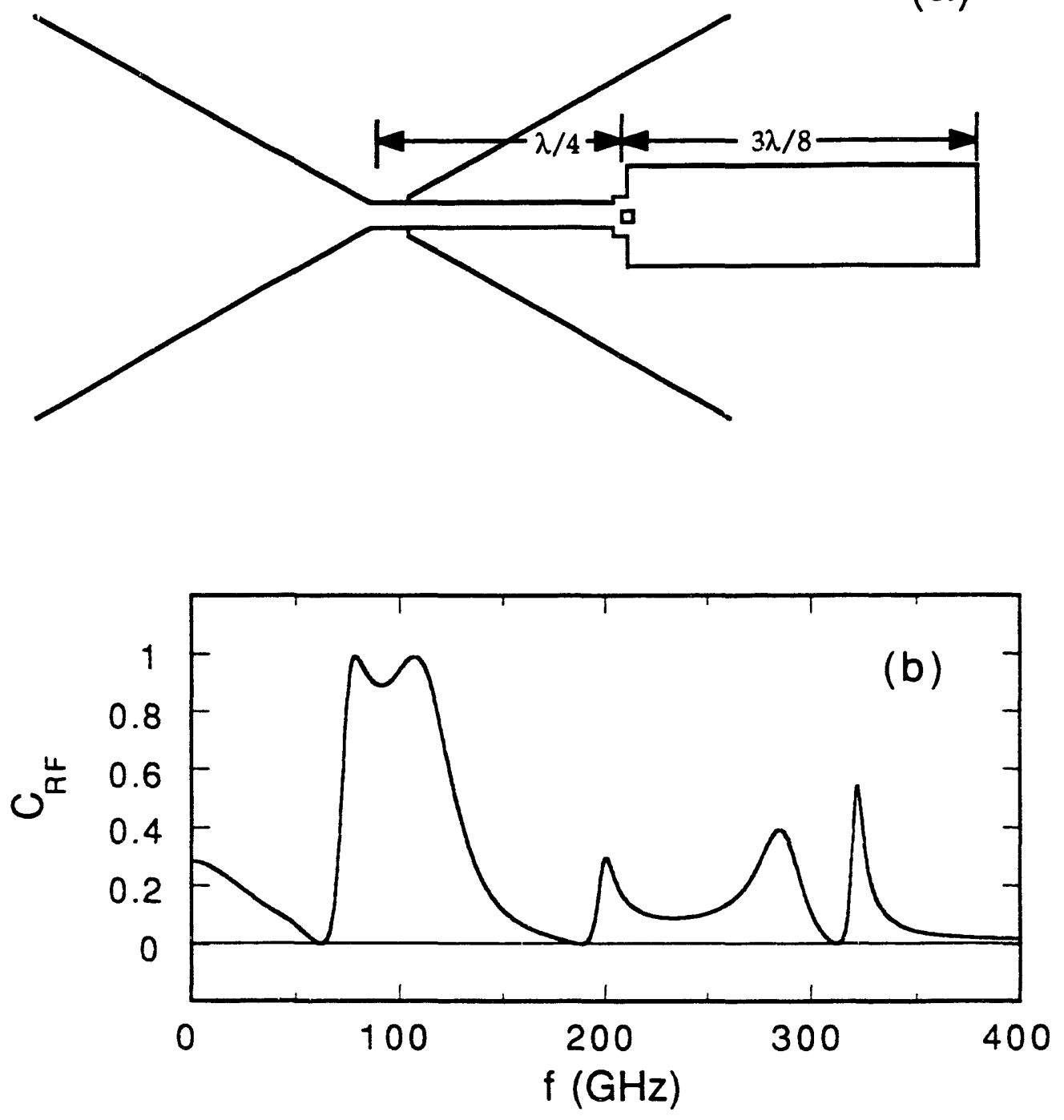

Fig 6.11 a) Diagram of a single SIS junction connected to the antenna terminals by a $\lambda / 4$ impedance transformer. The capacitance of the junction is resonated by a $3 \lambda / 8$ open-ended microstrip stub. b) Computed RF coupling coefficient for the structure shown in a). 

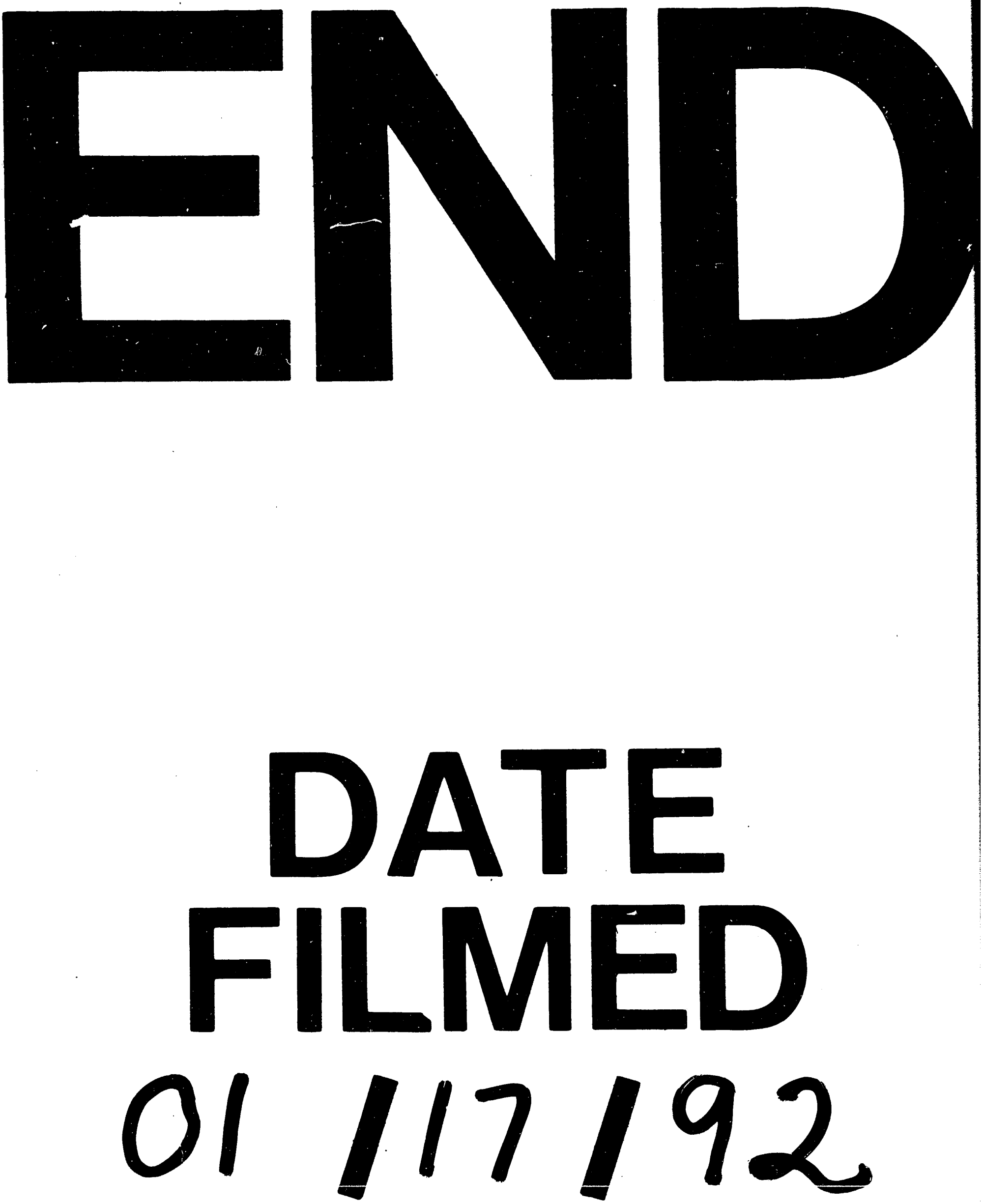
